\title{
Stegomalware: A Systematic Survey of Malware Hiding and Detection in Images, Machine Learning Models and Research Challenges
}

This paper was downloaded from TechRxiv (https://www.techrxiv.org).

\section{LICENSE}

CC BY 4.0

SUBMISSION DATE / POSTED DATE

06-10-2021 / 12-10-2021

\section{CITATION}

chaganti, Raj; R, vinayakumar; Alazab, Mamoun; Pham, Tuan (2021): Stegomalware: A Systematic Survey of Malware Hiding and Detection in Images, Machine Learning Models and Research Challenges. TechRxiv. Preprint. https://doi.org/10.36227/techrxiv.16755457.v1

$\mathrm{DOI}$ 


\title{
Stegomalware: A Systematic Survey of Malware Hiding and Detection in Images, Machine Learning Models and Research Challenges
}

\author{
Rajasekhar Chaganti, Vinayakumar Ravi, Mamoun Alazab, Tuan D. Pham
}

\begin{abstract}
Malware distribution to the victim network is commonly performed through file attachments in phishing email or downloading illegitimate files from the internet, when the victim interacts with the source of infection. To detect and prevent the malware distribution in the victim machine, the existing end device security applications may leverage sophisticated techniques such as signature-based or anomaly-based, machine learning techniques. The well-known file formats Portable Executable (PE) for Windows and Executable and Linkable Format (ELF) for Linux based operating system are used for malware analysis and the malware detection capabilities of these files has been well advanced for real time detection. But the malware payload hiding in multimedia like cover images using steganography detection has been a challenge for enterprises, as these are rarely seen and usually act as a stager in sophisticated attacks. In this article, to our knowledge, we are the first to try to address the knowledge gap between the current progress in image steganography and steganalysis academic research focusing on data hiding and the review of the stegomalware (malware payload hiding in images) targeting enterprises with cyberattacks current status. We present the stegomalware history, generation tools, file format specification description. Based on our findings, we perform the detail review of the image steganography techniques including the recent Generative Adversarial Networks (GAN) based models and the image steganalysis methods including the Deep Learning (DL) models for hiding data detection. Additionally, the stegomalware detection framework for enterprise is proposed for anomaly based stegomalware detection emphasizing the architecture details for different network environments. Finally, the research opportunities and challenges in stegomalware generation and detection are presented based on our findings.
\end{abstract}

Index Terms-Cybersecurity, Malware hiding, Multimedia Security, Steganography, Steganalysis, Image Features, Deep learning, Adversarial Generative Network,

\section{INTRODUCTION}

W ITH the proliferation of the internet availability to everyone at affordable costs and users can communicate through Internet to even remote locations, enormous amount of data is being generated and exchanged between the public

Rajasekhar Chaganti was with Dept. of Computer Science, University of Texas at San Antonio, San Antonio, Texas 78249, USA. e-mail: (Raj.chaganti2@gmail.com)

Vinayakumar Ravi was with Center for Artificial Intelligence, Prince Mohammad Bin Fahd University, Khobar, Saudi Arabia. e-mail: (vravi@pmu.edu.sa)

Mamoun Alazab was with College of Engineering, IT and Environment, Charles Darwin University, Darwin, NT 0820 Australia (e-mail: alazab.m@ieee.org)

Tuan D. Pham was with Center for Artificial Intelligence, Prince Mohammad Bin Fahd University, Khobar 34754 Saudi Arabia (e-mail: tpham@pmu.edu.sa) and private organizations, service providers, government, customers, small and medium businesses and other entities on a daily basis. The security and privacy of the user data has been a concern for a long time [1]. Cryptography is one of the way to improve the security and privacy when the user transmit the data through wired or wireless communication channels. The asymmetric and symmetric key combination techniques like SSL/TLS protocols developed to securely transmit the data between two endpoints. These protocols protect the original data being tampered and satisfies the security property "integrity". But, an adversary can still able to read the scrambled data in the communication channel if the man in the middle attack is performed and could apply the cryptanalysis to decipher the data [2]. Additionally, the secret keys may be exposed to the public or compromised by the adversary with poor security practices and lack of security awareness. Information hiding is another way to securely share the information through communication channels to others with no exposure of the secret information. In contrast to cryptography, the data is concealed in multimedia files in information hiding and no normal user is able to know that the information is hidden in the multimedia files. The information hiding techniques further classified into two types such as watermarking and steganography [3]. Watermarking is used for protecting and claiming the ownership of the digital assets, copyrights and intellectual property rights. It is most widely accepted to use for digital assets protection.

The another information hiding technique is the steganography. Steganography is the process of embedding the secret information into the cover medium for information hiding. The cover medium usually any multimedia file such as image, audio, video, text to hide the information. Anyone may use the steganography techniques to secretly share the data for legitimate purpose. For instance, government may share the confidential information with shareholders secretly using steganography. Despite steganography will improve the user data sharing privacy, the adversaries constantly look up new attacking ways and may be used steganography for illegitimate attack purpose. For example, an adversary may inject the malicious payload into the cover medium to evade the antimalware solutions detection. The malware hiding in the cover medium is termed as "stegomalware". We herein use the term stegomalware in the context of the malware hiding in multimedia files. The stegomalware can be incorporated in cyberattack life cycle to fool the security defender tools and perform the intended malicious activity. For instance, the lok- 
ibot malicious source code is concealed in the PNG image file for malware installation and avoid the detection of the malware by email security tools when distributing through phishing email campaigns [4]. The image files were used for malicious code obfuscation and sophisticated malware propagation in victim network. So, the stego malware detection technology is highly desired to identify the concealed data in cover medium for protecting the enterprise's assets and preventing the users for not becoming a victim of data breaches.

The multimedia file formats image, audio, video, and text are known to be considered for concealing the malicious content in enterprise attacks [5] [6]. The network protocols like TCP, UDP, and ICMP data format may be leveraged to hide the victim data and send over through network communication medium for covertly exfiltrating the data in the text form. The detection of this covert communication requires the decryption of the data packets and monitor the packet header or data anomalies for identification. These are difficult to detect in enterprise environment, as the enormous amount of network outbound traffic generates and may arise performance issues of decrypting the network traffic. Another category of attacks may use the image, audio, and video files as cover media to deliver the malware payloads and artifacts in the enterprise networks. These stegomalware may not be easy to detect and identify using existing enterprise security tools due to the complex nature of malicious payload hidden in the multimedia files and the tools are not capable of handling image, audio files for malware detection. The proactive mitigation plan like blocking the delivery of the cover media file formats is not a viable solution, as these file formats are extensively used in enterprise network for business transactions and can cost halting the business operations. So, an efficient, robust and accurate detection methods are needed to effectively identify these threats. Our analysis on the stegomalware history showed that images are extensively used for malware hiding. So, we focus on handling stegomalware in images in this paper.

Image steganography techniques are mainly categorized into spatial or transform domain based and spread spectrum techniques [7]. Recently, the GAN based image steganography solutions are popular and effective to hide the secret data [8]. In spatial domain, the techniques applied directly on the images to manipulate the pixels and store the secret information. On the other hand, in transform domain, the image file is transformed into transform domains such as DCT or DWT, and then secret information can be embedded in the DCT or DWT coefficients of the image[7]. Some of the methods may use the combination of spatial and transform domain to hide the secrets. Additionally, spread spectrum technique can be used to embed the secret information in the noise and then add the noise to the original media. GAN based steganography typically includes the generator, discriminator and steganalysis modules to iteratively generate stego images such that minimize the distortion between stego and cover image. These techniques can also be used by adversaries to hide the malware payload in images. So, the steganalysis methods should be applied to detect the hidden malware payload in images.

There are five different ways to perform the steganalysis.
Those are visual steganalysis, statistical steganalysis, signature based steganalysis, spread spectrum steganalysis, transform domain steganalysis and the universal or blind steganalysis [9]. In visual steganalysis, the hidden information on the images can be determined using the naked eyes. There are no techniques need to be applied for deciphering the hidden secret in visual steganalysis. We may use statistical, and signature based steganalysis for detecting the hidden information and possibly revealing the malicious intent of the file if it is part of attack propagation.

The signature based steganalysis focused on matching the known hidden secret technique patterns such as byte patterns, known text signatures for detection. The signaturebased detection can be using the existing forensic tools. These techniques may identify the files, which used the existing steganography techniques. However, a small change in the cover image or using advanced steganography algorithms like UNIWARD [10], HILL [11], WOW [12] makes the signaturebased techniques difficult to classify the stegomalware. Statistical steganalysis look for the statistical properties of the file such as entropy of Exif header, histogram of the pixel's distribution in the images and other attributes information to find the anomalies in the files when compared with the original files [9]. Spread spectrum steganalysis and transform domain steganalysis emphasize the importance of performing steganalysis indirectly on the image to improve the security and identify the classification even if the cover image is compressed, resized, and added with noise. The universal steganalysis techniques such as ML or DL based solution techniques can be applied to learn the behavior of the image using statistical features or unique feature taken from the sample data [13] [14]. But a proper selection of universal features for accurate classification is a challenge. The steganalysis classification is even harder when the dataset sample contain multiple image formats and want to obtain better detection performances.

Despite number of steganalysis techniques exist to detect the secret data, enterprises face challenges to identify the stegomalware because of lack of detection capabilities within the existing security tools deployed in the environment, the state-of-the-art detection accuracies are not enough to use in production environment [15], [16] lack of professional resource and difficulty of analyzing the large number of files traverse through enterprise. For instance, the malicious data embedded in an image may target the employees to compromise their credentials or install a backdoor code hidden in the image to establish a command-and-control (C\&C) server communication or send a suspicious attachment in the email to let the victim download into the machine and constantly communicate with $\mathrm{C} \& \mathrm{C}$ for data exfiltration.

Our state-of-the-art analysis shows that the research done so far considered the image steganography as an application to hide the data. Similarly, the image steganalysis is considered as a stego data detection tool. But, to our knowledge, there is limited work on exploring the malware hiding and detection in multimedia, specific to hiding in cover images, even though stegomalware is touted as one of sophisticated technique used by adversary in cyberattacks to evade the security detection 
[17] [18] [19]. This gap could be because of Image steganography and steganalysis, and cybersecurity are considered to be two different technical fields. We wanted to bridge the knowledge gap between these fields by discussing the current status of the stegomalware and presenting the current state of the art in image steganography and steganalysis. The stegomalware discussion includes history of stegomalware used in cyberattacks, stegomalware generation tools, stegomalware hiding multimedia file format specifications and the proposed stegomalware detection framework deploying in datacenter, AWS cloud and multicloud environment for enterprises. Based on our stegomalware history study, we have determined that the image is actively used as a cover medium for hiding malware content. So, we have preformed a detailed review of the image steganography and steganalysis techniques. The image steganography review includes describing image domain feature based solutions and GAN based solutions for steganography covering major research contributions in the state of the art. The image steganalysis review includes statistical feature and ML based solutions in spatial and JPEG domain, Rich models with Ensemble classifier and Deep learning based steganalysis solutions. To the end, we discuss the research challenges and directions on stegomalware hiding and detection techniques in the future. The main contributions of this work are as follows

- We discuss the current state-of-the-art stegomalware emphasizing the evidence based approach (stegomalware history, tools and file formats) with focus on recent attack trends.

- We perform a detailed review of image steganography and steganalysis techniques used for data hiding in images highlighting the major contributions for the last decades in chronological order and investigating the stegomalware contributions towards either image steganography or steganalysis.

- We provide a thorough review of GAN based image steganography for hiding the data securely and deep learning based image steganalysis for effective detection of the secret data, and also presented the performance comparison of the GAN based steganography and DL based steganalysis solutions.

- We present an enterprise stegomalware detection framework for identifying the stegomalware in multimedia, especially hiding malware in images and provided the framework implementation architecture for datacenter, AWS cloud and multicloud network environments.

- We also describe the research opportunities and challenges in the stegomalware hiding and detection solutions space by analyzing the stegomalware trends and reviewing the existing image steganography and steganalysis solutions.

The rest of the paper is organized as follows. Section II describes our motivation and aim of this study. Section III) compares the related work with our study. Section IV discusses the stego malware history of using it for malicious purpose. Section $\mathrm{V}$ illustrates the most familiar tools used for steganography to hide the content in multimedia. Section VI describes the various image and audio file formats used in malware activity. Section VII includes the review of the image steganography algorithms and their performances. Section VIII illustrates the steganalysis techniques used for image steganography detection. Section $\mathrm{IX}$ present the proposed enterprise framework for stegomalware detection. Section $\mathrm{X}$ includes the datasets used for performing experiments in steganography and steganalysis space. Section $\mathrm{XI}$ describes the various performance metrics used to assess the detection techniques and hiding algorithms in the prior art. Section XII discusses the research opportunities and challenges based on our study. Section XIII concludes the paper.

\section{Aim And Motivation}

The traditional antivirus solutions existed for a long time to protect the users and enterprise customers from known malware by incorporating signature and anomaly based detection techniques in the tools. As the adversary look for new ways to easily evade the traditional antivirus solutions, enterprises started adapting ML/AI based next generation end point security solutions. These solutions possess advances data analytics capabilities to detect not only known malware but also sophisticated malware variants. However, the antivirus and next generation endpoint solutions still exhibits false negatives due to the adversary usage of advanced techniques for hiding the malware and evading the detection. In particular, the enterprise security solutions find it difficult to detect the malware hidden in multimedia format files such as image, audio file formats. The existing solutions are designed to mainly focus on the signature and behavior analytics of the executable files for the malware identification. Additionally, the number of known malware samples hidden in multimedia file format are very less for applying the ML/DL techniques and predict the malware files.

Security professionals may find it even difficult to perform stegomalware mitigation activities such as blocking file formats at Firewall, Intrusion Detection/Prevention System or endpoint security level, as the image, audio and video files formats are extensively used in the enterprise for business operations and transactions. So, the security workforce most likely may ignore to perform any proactive actions on stegomalware by considering the criticality of not allowing multimedia format in the enterprise for business continuation except reacting when the device compromise or data breach is identified. Additionally, the file signature update in security tools also may not be a viable option, as the malware constantly evolves to evade the detection. Furthermore, the malware hidden image, audio files are inherently complex to identify because of no suspicion, various file formats and structures to process and characterize the malware using the state-of-the-art security products. Based on these facts, it is clear that adversary using multimedia formats such as image, audio to hide the malicious executable, malicious IP or C\&C server domain name detection is challenging and there is no existing solution to claim to be effectively and accurately detecting these stegomalware files in enterprise organizations.

Our motivation of this study is to identify the technology gaps and reasons for not addressing the stegomalware problem 
actively so far, address the stegomalware by investigating the image steganography state-of-the art solutions since 2000, explore the cross discipline technical field dependencies to envision the effective solution space for stegomalware detection in enterprises. Interestingly, there is limited work in recent times exploring the stegomalware hiding in multimedia topic [20] [21] [22], even though great amount of research being done discussing the steganography and steganalysis techniques in multimedia files. The reasons could be the topic is multidisciplinary field focussing on the signal and image processing, cybersecurity and malware analysis. So, another reason of motivation to this study is performing a thorough study considering the industry needs and academic research current status.

The main aim of this study is multifold. Firstly, an adversary adapts stegomalware techniques nowadays for evading the detection. So, we wanted to present an overview of the stegomalware, including the stegomalware history, tools use for hiding malware, multimedia file for malware hiding format specifications, steganography and steganalysis techniques in data hiding. We believe that our study will enable the cybersecurity workforce to explore the prior art and build solutions to detect stegomalware. For instance, our study on the steganalysis using deep learning techniques in this paper can be used as a reference to train and test the models for image based stegomalware detection and implement the production level effective stegomalware solutions if optimal solution available in the state of the art. Secondly, our study is seen as a reference for bridging the knowledge gap between the signal and image processing researchers, deep learning/GAN specialists and the cybersecurity technologists especially malware researchers to continue performing research in unfathomed malware hiding in multimedia file formats. Furthermore, we wanted to explore and discuss the research challenges for detecting and mitigating the stegomalware.

\section{RELATED WORK}

In this section, we have described the relevant prior art works in information hiding specific to image as a cover and their contributions. Researchers reviewed various aspects of the steganography and steganalysis mainly focused on the generic data hiding using multimedia file formats as a cover. But, as our study on the stegomalware history reveals that adversary mostly leverage images as a cover to hide the malware or malicious artifacts, We restrain our scope of study to image based steganography and steganalysis even for related work comparison. The stegomalware covert channel communications hiding in network protocol stack i.e. text based hiding stegomalware [23] [24] [25] also not considered in our study because the enterprises could decrypt the network traffic and monitor for anomalies to identify cover channels and may prevent stegomalware spread. The image steganography is further divided based on the spatial or transform domain algorithms used for data hiding in images [7] and GAN models [26] used to generate stego images. The steganalysis is further classified based on the combination of the rich models for feature extraction and ML models for classification [9], and the application of deep learning models for steganography detection [27].

Mazurczyk et al. [28] performed a survey on the steganography techniques and mitigation solution on the smartphones in 2015. The authors emphasized the malware attacks leveraging steganography to hide malware on smartphone, discussed the steganography tools, steganography techniques in the context of feature domain and ML based solutions, mitigation solutions mainly focusing to cover channel communication mitigation. However, the paper did not cover the recent advancements of using GAN to generate stegomalware and deep learning models for efficient detection stego content instead of rich models with classification techniques. Furthermore, the paper survey is limited to smartphones, which may not cover the enterprise end point based study. We also described the datasets used in the state-of-art, performance metrics needed to evaluate new models if someone wanted to evaluate them and file format specification to explore the opportunities for analyzing the specifications either for steganalysis or for steganography for secured hiding. We believe that the paper [28] is somewhat close to our study topic exploring the stegomalware in multimedia.

Other review studies mainly focused on a particular aspect of image steganography either focusing on the prior art work review in particular domain features [29] [30] [7] [31] [32] or review on the GAN models [26] [33] [34]. Some works reviewed the steganalysis detection techniques using rich models or domain based extractions along with Machine learning models [9] [32] and other works mainly reviewed deep learning based steganalysis detection techniques [27] [35] [36]. None of these works provided a holistic overview of the information hiding and hidden data detection particularly in the context of the hiding malware in images. So, we could see that our work can be good reference to continue research either steganography or steganalysis particularly using deep learning or GAN models and most importantly in the context of enterprise security for advanced stegomalware detection.

\section{Stegomalware History}

We discuss the existing malware families and their behavior when leveraging the steganography on multimedia files to hide malicious commands or source code. The steganography technique is rarely seen in malware generation. However, this technique is used to create sophisticated malware for organized crimes like operated by APT groups and hard to detect those malware attempts. So, it is indeed very important to detect the stegomalware targeting the enterprises and other sectors. The APT malware "Operation shady RAT" was first seen to be using digital images to hide the command $\mathrm{C} \& \mathrm{C}$ server addresses in 2011 and use it to connect the C\&C servers from the compromised victim machines. The malware spread through targeted phishing email campaign and compromise the machine when the user downloaded the attached file from the email [38]. NSS lab researchers discovered that the duqu malware targeting the industrial manufacturers has steganography process to embed the victim data into an image file prior to sending to the attacker controller servers. This is 


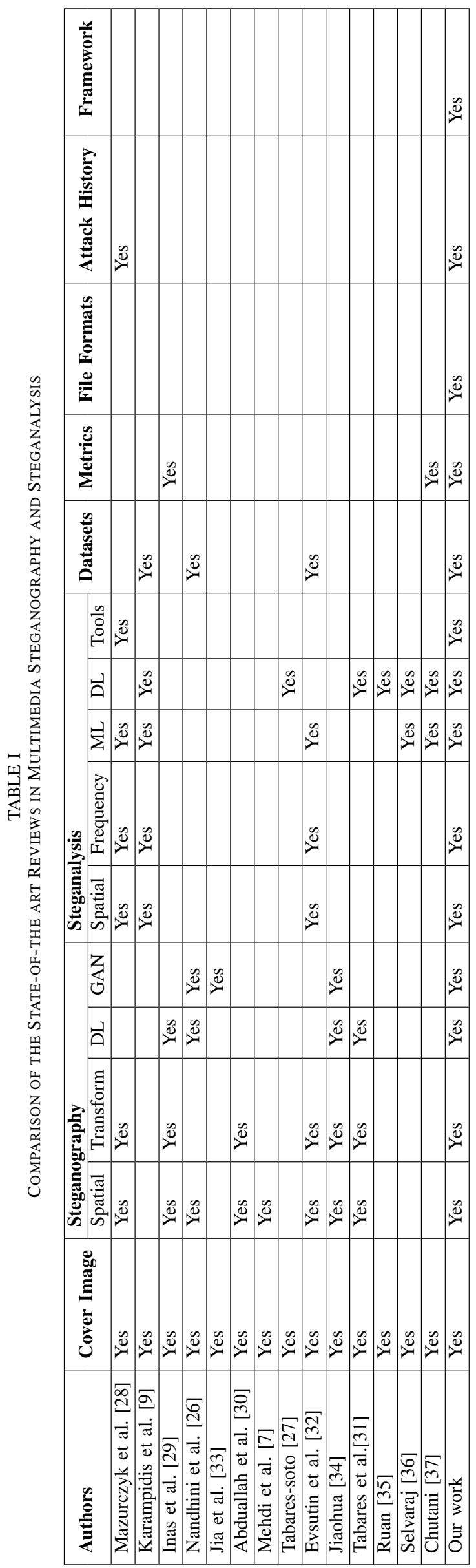

TABLE II

DESCRIPTION OF THE ABBREVIATIONS

\begin{tabular}{|c|c|}
\hline Acronym & Description \\
\hline ACGAN & Auxiliary Classifier GAN \\
\hline ANOVA & analysis of variance \\
\hline ASDL & Automatic steganographic distortion learning \\
\hline APT & Advanced Persistent Threat \\
\hline BSM & Binary Similarity Measures \\
\hline BMP & Bitmap \\
\hline $\mathrm{BBC}$ & Block Boundary Continuity \\
\hline & Convolutional Neural Networks \\
\hline CRM & $\begin{array}{l}\text { color rich model } \\
\text { Discrete }\end{array}$ \\
\hline CWT & $\begin{array}{l}\text { Discrete Cosine Transform } \\
\text { Complex Wavelet Transform }\end{array}$ \\
\hline DFT & Discrete frequency transform \\
\hline & Define Restart Interval \\
\hline DTCWT & Dual Tree Complex Wavelet Transform \\
\hline & Discrete Wavelet Transform \\
\hline DQT & Define Quantization table \\
\hline DBN & Deep Brief Network \\
\hline DNS & Domain Name Server \\
\hline DAT & $\begin{array}{l}\text { Discrete Cosine Transform Residual } \\
\text { Define Arithmetic Coding }\end{array}$ \\
\hline DCTR & discrete cosine transform residual \\
\hline DHL & $\begin{array}{l}\text { Denne Huitman lable } \\
\text { Dynamic Link Library }\end{array}$ \\
\hline ELF & Executable and Linkable Format \\
\hline & Ensemble Classifier \\
\hline EA & Edge Adaptive \\
\hline GIF & Graphics Interchange Format \\
\hline GFR & Gabor filter Residual \\
\hline $\begin{array}{l}\text { GNCNN } \\
\text { GRDH }\end{array}$ & $\begin{array}{l}\text { Gaussian-Neuron CNN } \\
\text { Generative reversible data hiding }\end{array}$ \\
\hline & Generative Adversial Networks \\
\hline HUGO & High Undetectable steGO \\
\hline Hidden & Hiding data with deep networks \\
\hline HIGAN & Hiding images GAN \\
\hline HILL & High-pass, Low-pass, and Low-pass \\
\hline IWT & Integer Wavelet Transform \\
\hline ICMP & Internet Control Message Protocol \\
\hline IUERD & Improved UERD \\
\hline JPEG & Joint Photographic Experts Group \\
\hline JRM & JPEG Rich model \\
\hline $\mathrm{LSB}$ & Least Significant Bit \\
\hline LSER & enhanced residual \\
\hline ML & $\mathrm{Mac}$ \\
\hline MME & Modified Matrix Encoding \\
\hline MiPOD & Minimizing the Power of Optimal Detector \\
\hline MPEG & Moving Picture Experts Group \\
\hline MSB & Most significant bit \\
\hline NPQ & Normalized Perturbed Quantization \\
\hline $\mathrm{PE}$ & Por \\
\hline PCM & Ulation \\
\hline PNG & k Graphics \\
\hline PPM & Pixel Pair Ma \\
\hline PQt & Perturbed Quantization \\
\hline PVD & $\mathrm{Pi}$ \\
\hline $\mathrm{PQ}$ & Pertl \\
\hline PHARM & Model \\
\hline PRSM & iodel \\
\hline QMF & \\
\hline RIFF & Resource Interchange File Format \\
\hline RNN & Recurrent Neural Network \\
\hline & Rectified Line \\
\hline $\mathrm{RBF}$ & Radial Basis \\
\hline SVM & Support Vector Machine \\
\hline SPAM & d-order Markov chains \\
\hline SEGAN & Steganographic Encryption GAN \\
\hline SSGAN & Steganography Based on GAI \\
\hline SPAM & ncy Model \\
\hline SGF & $\begin{array}{lll}\text { stee } \\
\text { Sng }\end{array}$ \\
\hline & Rich Model \\
\hline SGAN & Steganographic GAN \\
\hline SCA & Selective Channel Aware \\
\hline TIFF & Tagged Image File Format \\
\hline 120 & trui \\
\hline $\begin{array}{l}\text { TCP } \\
\text { UDP }\end{array}$ & $\begin{array}{l}\text { Transmission Control Protocol } \\
\text { User Datagram Protocol }\end{array}$ \\
\hline WA & Waveform Audio File Format \\
\hline WGAI & 1 GAN \\
\hline WOW & Wavelet Obtained Weights \\
\hline UNIWA & \\
\hline UED & \\
\hline UERD & Un \\
\hline
\end{tabular}


a clear indication that the image file can be used to hide the secret content and send through the internet in image form so that network level detection mechanisms not able to detect the data exfiltration [39].

Zeus variant Trojan used for stealing money from victim bank account incorporates the configuration file in the image for deceiving the enterprise security tools. The configuration file may include the list of bank details, malicious code to steal the online banking details and hijacking the login details to the attackers. To conceal the configuration file, the encoding and symmetric encryption techniques applied on the cover image [40]. In [41], the authors describe that the Gatek/stegoloader encrypts the shellcode and hidden in the digital image. The shellcode contains bot command to retrieve web browsing history, victim system information, executing payload and retrieving the software installed in the machine. When the malware installed in the victim machine, it connects to the $\mathrm{C} \& \mathrm{C}$ server to download the stegomalware into the machine. Then, decrypt the shellcode from the image to run bot commands and exfiltrate the victim data. TeslaCrypt is a ransomware Trojan to encrypt the victim data once the targeted machine is compromised and expect ransom to receive the decryption key. Steganography is used to conceal the C\&C server commands in the cover image [42]. The stegomalware images displayed on the website ads click using the web browser may be enough to infect the victim machine. Stegosploit malware exploit can leverage the HTML5 < canvas> tag to make the browser read the pixel data as JavaScript and execute the malware functionality. This technique can be used to exfiltrate the machine data to the attacker controlled server [43]. Cerber Ransomware uses the steganography image to store the malicious executable code. So, when the victim clicks on the phishing email delivered with malicious word document, the downloaded document runs the macro code. This code loads a steganographic image to run the malicious executable code and perform the encryption of all the data on the machine [6].

The stegomalware is also extensively being used recently to target the users visiting websites. For instance, the "stegano" exploit conceal the malicious code in the pixel of banner advertisements on websites. When a user clicks on the advertisements, the malicious code run on the machine and may install the DNSchanger to change the local DNS IP address. From then onwards, the victim machine directs the DNS requests to the attacker-controlled DNS server. An attacker may redirect the victim to the phishing website to install drive-by download malware and more, when the victim perform a legitimate request to trusted sites [44]. AdGholas group targeted UK universities infecting with ransomware in 2017. Further investigation on the infection revealed that the maladvertising campaign involves stegano exploit for the widespread infection of the ransomware [45]. These real time attack instances portray the stegomalware infection through maladvertisements has a large scale widespread across the nations. Sundown pirate exploit kit also use stegomalware hiding the vulnerabilities of popular application in the cover image to test against the victim machine and execute the vulnerabilities code to infect the victim machine. When the user visits a website, the maladvertisements has iframe links to redirect them into malicious URL hosting a white image and download the image into the victim machine. The white image is a stegomalware, which can infect the machine with known vulnerabilities in popular application [46].

A researcher from Emmisoft discovered that Synccrypt ransomware distributing the malicious code zip file using steganographic JPEG images. The attack vector consists of sending phishing emails with attached windows script file (WSF); when the user run the script, it downloads the JPG stegomalware malware with payload embedded in the zip format and extract the ransomware components from the zip file so that it can create windows scheduled task to run the ransomware for encrypting the victim data [47]. ZeroT Trojan malware mainly used by China espionage group reportedly using PlugX RAT module to infect the targeted industries. The infection process involves sending a phishing email with malicious doc file as an attachment. If the victim user opens the document and agrees to run the hidden malicious application, the malware application is installed on the victim machine. Then, the malware connects to $\mathrm{C} \& \mathrm{C}$ server to exfiltrate the user data and downloads the stegomalware image containing the PlugX RAT payload for performing malicious activities on the victim machine.

Recently, shlayer adware is extensively used by adversary to infect the Mac machines. An adversary usually tricks the user to install fake adobe player for downloading the shlayer adware. A recent maladvertisements campaign noted to be downloaded by 5 million users in a day, which contains the stegomalware as part of the attack lifecycle. The stegomalware image hide the malicious URL used for downloading the fake adobe player. An HTML5 object run through the image when user click on the advertisements and in each loop, it extracts a malicious URL character. Once the loop is completed, all the extracted characters clubbed together to form the malicious URL and then redirects to the malicious website hosting fake player [17]. The audio files are also considered as a cover media for performing malicious activity. Waterbug cyber espionage group utilizing the audio file format WAV to conceal the malicious DLL files for installing on the victim machine during the attack lifecycle [49].

Table III illustrates the list of malware seen in the history using stegomalware to hide their activity or download the malicious code without being detected by anti-malware tools. Based on the description of the role of stegomalware in famous malicious campaigns and the collection of attacks in Table III. we can observe that most of the malware leverage the image files formats for carrying the payload and occasionally seen video and audio formats as a payload carrier. The malware infection in the history shows that these stegomalware are targeting multiple industries and particularly used to evade the malware detection by Deep Packet Inspection (DPI) or endpoint security tool detection capabilities. Furthermore, we identify that the stegomalware is mostly seen to conceal the malware payload, exfiltrate the data in the form of steganographic multimedia files and store the $\mathrm{C} \& \mathrm{C}$ IP address, domain name and the commands to communicate with the attacker server. Overall, it is evident that the stegomalware is popularly 
TABLE III

HISTORICAL MALWARE VARIANTS EMPLOYING STEGANOGRAPHY FOR EXPLOITATION

\begin{tabular}{|c|c|c|c|c|c|}
\hline Malware & Technique & Exploitation Stage & Targeted Industry & File Type & File Format \\
\hline Operation Shady RAT [38] & Phishing email & $\mathrm{C} \& \mathrm{C}$ server connection domains & Government, International Corp, Nonprofit & Image & Not known \\
\hline Duqu [39] & Installing Rootkit & Data exfiltration & Industries & Image & Jpg \\
\hline ZeusVM 40] & Maladvertising campaign & Exfiltration & Banking sector & Image & Jpeg \\
\hline Gatak/ Stegoloader [41] & Hosting malicious image in legit website & Download malware & - & Image & Png \\
\hline teslacrypt [42] & Browsing malicious Page & Download C\&C commands & Generic Internet users & Image & Jpeg \\
\hline Stegosploit [43] & Leverage HTML5 canvas tag & Download malicious code & Internet users & Image & Not known \\
\hline Cerber 6 & Phishing email & Malware Delivery & Various sectors & image & Jpeg \\
\hline DNSchanger 44] & Advertisements & Malware Delivery & Internet Users running vulnerable Routers & Image & Png \\
\hline Vawtrak [5] & Hide in Favicon Icon & Download malware & Internet users & Image & Favicon icon \\
\hline AdGholas 45] & Maladvertising Campaign & Exploitation & Education, Travel & image & jpeg \\
\hline Sundown [46] & Malvertising campaign & Exfiltration & Internet users & Image & Png \\
\hline Synccrypt 47] & Click Malicious URL & Install malware & Generic Internet users & Image & Jpeg \\
\hline ZeroT [48] & Phishing Campaign & Command and Control & Not known the target & Image & Bmp \\
\hline Verymal 17$]$ & Maladvertising & Downloading Shlayer Malware & Internet users & Image & Jpeg \\
\hline Waterbug 49] & Legitimate application vulnerabilities & Downloading DLL & Government, Education, IT & Audio & Wav \\
\hline Loki Bot [4] & Phishing emails & Install malware & Internet users & Image, Video & Jpg, Video formats \\
\hline
\end{tabular}

used by cyber espionage groups as part of attack propagation, even though it is not frequently touted in malware industry.

Lesson Learned: Our stegomalware history review clearly show that the stegomalware is used in many cyberattack campaigns to hide the artifacts or malware payloads and evade the security detection. We are convinced that cyberattacks using stegomalware to hide the activity will keep growing, and the organizations should monitor and adapt image file stegomalware detection solutions.

\section{Stegomalware Creation tools}

There are various techniques used to conceal the payload in the multimedia files based on the cover media type and payload type. Over the years, there were a number of tools developed to perform the compression, encryption and encode the payload so that the scrambled data can be inserted into the multimedia files. Table IV depicts the popular tool set used for performing steganography on the audio, image and video file formats but not limited to. As the image form as cover media is most used for hiding the content and deceiving the users, most of the existing tool's support to perform steganography in images. The most popular steganography tools steghide, openstego, Hide'N'Send, SSuite Piscel, Camouflage, Xiao, Openpuff are used for performing steganography and most of them supports JPEG, BMP file formats, as shown in Table IV Some of the tools Xiao, Steghide, Deepsound, Openpuff and Silenteye support for hiding the content in audio files like wav, $\mathrm{mp3}$. But few of the tools openpuff and camouflage supports for hiding the content in video file formats. We can observe that most of the tools supports windows operating system to hide content. The popular tool steghide has open source code available for analysis written in $\mathrm{C}++$ and using advanced LSB techniques for hiding the content. Openpuff offering the subscription-based solutions for steganography and provides advanced capabilities like multi-layered data obfuscation.

Lessons Learned: There are number of public tools available for performing steganography and hiding the malware payload or data. However, the advanced steganography solutions like UERD, UNIWARD, Mi-POD, HILL may not be available in in-built tools for generating stego images. Overall, the stegomalware generation using the existing tools is much easier and definitely a concern for organizations to defend stegomalware.

\section{STEgomalWARE MULTimedia FILE FORMats}

The stegomalware payload embedding in different multimedia file formats such as image, audio or video is illustrated in the Figure 1 . The cover image in the Figure 1 is embedded with malicious command and control server IP address payload using lease significant bit algorithm. During the attack progress, the malware triggers to retrieve the malicious IP address from the cover image and initiate outbound connection to that IP address for data filtration or other malicious activity. In cover audio file scenario, the command and control server domain name is embedded with audio using selective based embedding steganography algorithm. The same malware outbound communication process seen in cover image applicable for this scenario as well. In video cover steganography, the malware binary "evil.exe" is converted into hexadecimal representation and encoding based steganography is used to embed the malware binary in consequent frames of a video. As we have seen in Section IV] most of the stegomalware used image as a cover for hiding the malicious artifacts. So, we have decided to give a detailed description of image file format specifications here.

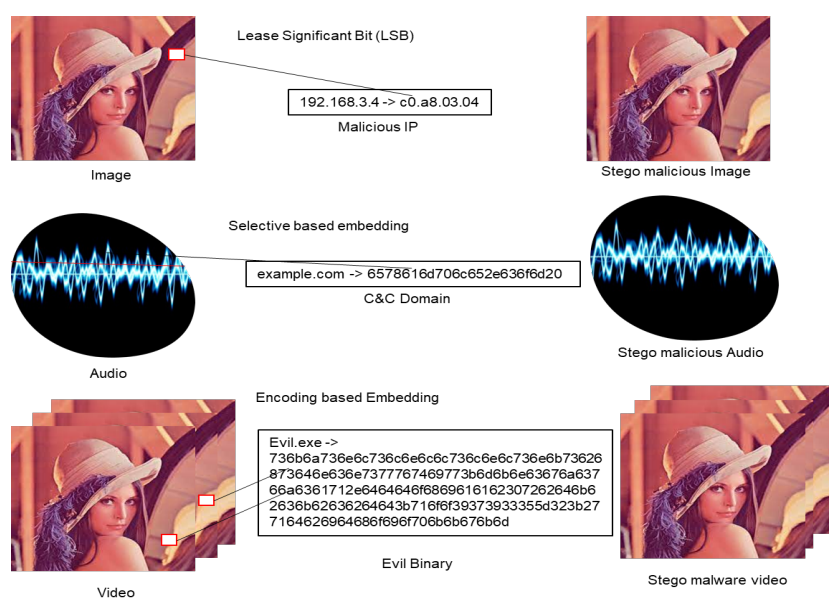

Fig. 1. Multimedia Stegomalware

The multimedia file formats used to hide the malicious payloads in the real time attacks seen over the last decade are JPG, BMP, GIF, PNG and WAV formats. The detail description of these file format structure is needed to understand 
TABLE IV

STEGANOGRAPHY TOOLS FOR MULTIMEDIA FILES

\begin{tabular}{|l|l|l|l|l|l|l|l|}
\hline Tool & Year & Last Update & OS & Image & Audio & Video & Supported Format \\
\hline Steghide[50] & 2003 & 2013 & Windows, Linux & Yes & Yes & No & Jpeg, Bmp, Wav and Au \\
\hline Openpuff [51] & 2004 & - & All Platforms & Yes & Yes & Yes & Bmp, Jpg, Png and more \\
\hline Xiao [52] & 2006 & 2007 & Windows & Yes & Yes & No & Bmp, Wav \\
\hline MP3stego [53] & 2006 & 2015 & Windows, Linux & No & Yes & No & Mp3 \\
\hline Crypture [54] & 2007 & 2007 & Windows, Linux & Yes & No & No & Bmp \\
\hline Our Secret [55] & 2008 & 2013 & Windows & Yes & No & No & Generic image file format \\
\hline SteganographX Plus [56] & 2010 & 2010 & Windows & Yes & No & No & Bmp \\
\hline Hide'N'Send [57] & 2012 & 2012 & Windows & Yes & No & No & Jpeg \\
\hline SSuite Piscel [58] & 2014 & 2018 & Windows & Yes & No & No & Bmp, Png \\
\hline SteganPEG [59] & 2014 & 2014 & Windows & Yes & No & No & Jpeg \\
\hline Silenteye [60] & 2014 & 2020 & All Platforms & Yes & Yes & No & Generic image and audio \\
\hline rSteg [61] & 2015 & 2015 & All Platforms & Yes & No & No & Png \\
\hline Openstego [62] & 2015 & 2020 & Windows and Linux & Yes & No & No & Bmp, Gif, Jpeg, Jpg, Png and Wbmp \\
\hline Outguess for mac [63] & 2017 & 2018 & Mac & Yes & No & No & Jpeg \\
\hline Deepsound 2.0 [64] & 2018 & 2018 & Windows & No & Yes & No & Wave, Flac \\
\hline
\end{tabular}

where the attackers hide the malicious content and see the possibilities identifying the file features to accurately detect the stegomalware in each of these file formats.

JPEG stands for Joint Photography Experts Group and the JPEG standard created in 1992. It represents the digital image in compressed form using the DCT algorithm and widely used standard for viewing the images in the digital devices. The JPEG images ends with the .jpg or .jpeg format [65].

The Figure 2 illustrates the different markers used to represent the image data in the JPEG structure format; the markers hexadecimal representation to identify the markers in the given image [66] and the Exif file format structure to store the image captures or processed device attributes and other information [67]. The JPEG markers categorization start with the value "0x FF02" and ends with 0x FFFE. The byte "FF" is not shown in the Figure 2.1 for simplification and good presentation purpose. The JPEG image usually starts with marker Start of the Image (SOI) data "0x FFD8" and ends with End of Image "0x FFD9". The encoded content in the image is stored inside the frames. Each frame typically contains more than one scan units to store the image content. A JPEG may contain up to 15 frames starting with the segment "Start of Frame" and having a hexadecimal values in between the range from 0xFFC0- 0xFFCF. The Start of Scan (SOS) is used to store the encoded image components and can be identified with the hexadecimal value 0x FFDA in the image. The JPEG images may also contain "Reserved" marker segment starts with $0 x F F 02-0 x F F B F$ to add additional information to the JPEG image. The Define Huffman Table (DHT), Define Quantization table (DQT), and the Define Arithmetic Coding (DAT) store the Huffman, Quantization and Arithmetic coding data used during the lossless compression of the JPEG image. These markers start with 0x FFC4, 0x FFDB and 0x FFCC are used to parse the image and extract the relevant data for decompressed image presentation. Some of the markers can be repeated based on the Define Restart Interval (DRI) in the JPEG image representation and these can be started with 0xFFD0-0xFFD7 having a naming convention Restart Marker 1 to 7. The Define Hierarchical Progression (DHP) and Expand Reference Component (ERC) are not commonly used markers and assigned the hexadecimal values 0xFFDE and $0 x F F D F$ respectively to identify them in the images. There are various JPEG extensions 0 -13 options defined in the JPEG specifications having a marker hex ranges 0xFFF0-0xFFFD. JPEG extension 7 is commonly used for lossless JPEG image representation. The JPEG specification also contain the application segments $0-15$ to add the application content in the image and can be identified with hex values 0xFFE0-0xFFEF.

The App1 segment 0xFFE1 is seen to be used for representing the Exif metadata, JPEG thumbnail and TIFF IFD format, when the image is taken from digital camera or the creation of image using the Adobe extensible metadata platform. The App1 data comprises the APP1 data size represented in bytes length, Exif Identification code, Tiff header, 0th Image File Descriptor (IFD), 1st IFD value and thumbnail image data for representing the Exif data in Tiff format. The data size is not a fixed value, and it depends on the other fields in the Exif format. To identify the Exif format, the Exif identified value is assigned as hex value "Exif". The tiff header contains 2bytes of byte align definition of the data, 2 bytes of Tag Mark "0x2A00" and the offset value to first IFD "0x00000008". The byte align values can be "I I" or "M M", which represents either Intel or Motorola byte align representation of the data. The 0th IFD represents the main information image data and the stored in the 0th file directory, and it links to the 1st IFD; the 1st IFD represents the IFD of thumbnail image data and the link is terminated. IFD0 may also contain the Exif SubIFD, which stores the digital cameras information. Exif format support three formats JPEG, RGB TIFF and YCbCr TIFF format to represent the thumbnail images. If the thumbnail image is saved in JPEG, then the structure of the JPEG follows the same structure format as shown in Figure 2. Having seen the typical JPEG file format specifications so far, there are many ways an adversary hides the malicious payload in JPEG images. In particular, the Exif data and thumbnail images storage give more space to embed the malware content. 


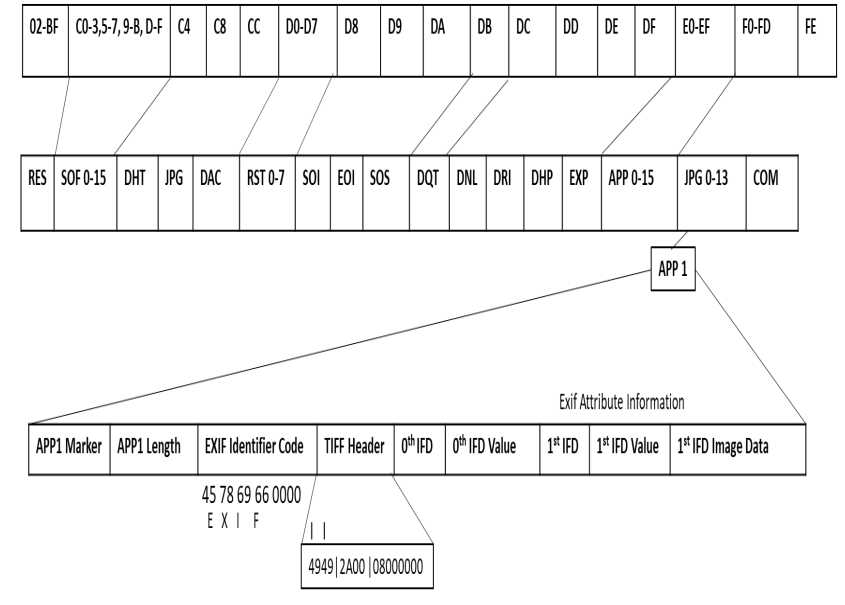

Fig. 2. JPEG and Exif file structure format

The PNG image file start with PNG hex decimal signature "89 $504 \mathrm{e} 47$ 0d 0a 1a 0a" and the byte sequence "0x50 0x4e $0 \times 47$ " represents in ASCII letters as "PNG" is used to identify the given image is PNG image or not. The value " 89 " is used to detect the transmission systems that do not support the 8-bit data and eliminating the chances of mistakenly interpreting the text file as PNG files. The last 4 bytes in the signature is used to detect the DOS-Unix line ending conversation of the data [68]. The PNG data is divided into chunks in the image. Each chunk has four fields as shown in the Figure 3 . The chunk data size is determined by the first 4 bytes assigned for representing the data length in bytes. The chunk types can be classified as Critical chunks and Ancillary chunks. The critical chunks must be presented in the image to read by the image readers. There are four chunks comes under critical chunks. Those are Image Header (IHDR), Palette (PLTE), Image data (IDAT), Image End (IEND). The Cyclic Redundancy check (CRC) is used to perform the integrity check on the chunk data for errors and data corruption. The four critical chunks are shown in the PNG specification format Figure 3 . The image header chunk type has a length of 13 bytes and contains the image metadata. The PNG image width and height values consumes the first 8 bytes of the image data field. The number of bits per palette or index represented by the one-byte bit depth. The one-byte color type signifies the grey scale or color of the images. The two bytes used for compression and filter method enables the image compression and filtering the image before compression if needed. The interlace method can have two values: no interlace and Adam 7 interlace. When no interlace is selected, the image is scanned from the top to bottom and the pixel scan starts from left to right sequentially. The Palette chunk may contain 1 to 256 palette entries and each entry can be represented in RGB image form. The number of entries can be selected based on the chunk length. The Image Data (IDAT) contains the image data processed using the meta data assigned in the Image header, which includes finding the size of the data and performing filtering and compressing the data. The Image End (IEND) chunk contains a single empty byte to mark the end of the PNG data stream. In addition to the critical chunks, the optional ancillary chunks like bKGD, sBIT, tIME can be embedded in the PNG image for conveying other information about the image. The detail description of the ancillary chunks can be found [69].
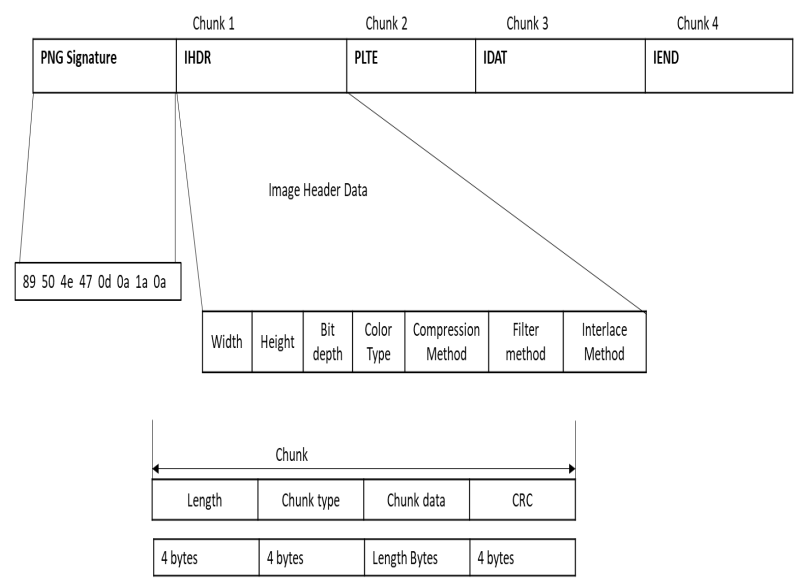

Fig. 3. PNG file structure format

The GIF image format ads can be used by adversary to host fake ads with embedded malicious URLs so that the victims are redirected to the malicious web pages when the user click the ad page. The first three bytes representing as "GIF" in hex decimal can be used to determine if the image is GIF or not. The next three bytes signifies the GIF version. As shown in Figure 4, the GIF image has version 89a in the image. These first 6 bytes forms the GIF image header block. The next block "Logical screen descriptor" is used for knowing the amount of space needed for the GIF image. The canvas width and height determine the image size and the pixel aspect ratio to set the proportions of the width and height. The packet field does contain multiple color flags like Sort flag, color resolution, Global color table flag and size global color table, which are used to set the global color aspects of the GIF image. If the global color table flag set in the logical screen descriptor, the global color table should be followed to specify the global color image configurations. The color table consists of the RGB color intensities represented color values in between 0 and 255. The GIF may use the global color table for all the sub images or local color table for each sub images in the GIF. Based on the color depth value mentioned in the logical screen descriptor, the global color table is determined and used for coloring the GIF canvas image. The graphical control extension is optional and varied in length. This block specifies the transparency settings and control the image animations. The first byte for extensions always starts with " 21 ", which is also called extension introducer and ends with block terminator " 00 ". The image descriptor block stores the local or sub images data and number of these blocks used if the number of sub images present in the GIF image. The first byte is an image separator and denoted by the hex value " $2 \mathrm{C}$ ". The next 8 bytes represents the position and size of the local image in the GIF image. The packet field contains multiple color related flags including the local color table flag. If the local color table flag is enabled, the next block can be local color table for representing the color aspects of the local 
image. If the local color table flag is not enabled, the global color table is used for the local image color. Then, the image data block stores the data in data sub-blocks. The image data block starts with LZW minimum code size " 02 " and the sub block first bytes signifies the length of the sub block. So, the next number of the bytes in the sub block are the real data to read until reaches the length of the bytes and then the next subblock length can be seen in the first byte and continue this procedure to read all data until the last sub block reaches to "00" block terminator. There are few extensions such as plaint text, application and comment extensions are used for specifying the text captions in the GIF image, embedding the application specific information in the GIF image and adding the ASCII text information to GIF image, respectively. These extension blocks are all optional to the GIF image. The image ends with trailer marker " $3 \mathrm{~B}$ " to confirm that the image is ended. However, an adversary may add the malicious payload after the trailer marker to hide the content and infect the victim machines when triggered in the scheduled action of items.

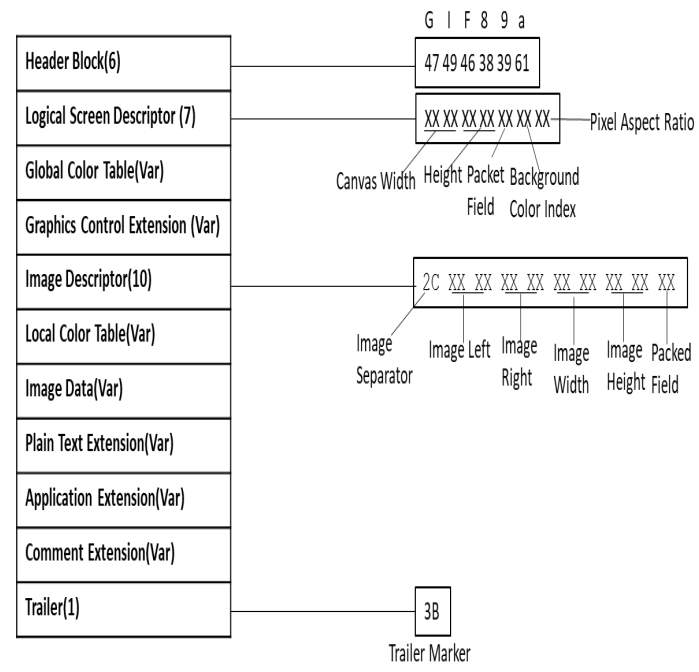

Fig. 4. GIF file structure format

The BMP file format is also called as device independent bitmap (DIB) image file format used to store the twodimensional images both in gray scale and color [70]. Microsoft defined the image representations with color resolutions and can still map the image colors with internal representations even when move the bitmap move from one device to another device. The BMP file starts with 14 bytes file header. The BMP signature hex value " $424 \mathrm{D}$ " is used to determine the BMP images and the next four bytes contains the file size. The following next 4 bytes are reserved and application specific. These fields usually unused and contain zero-byte values. The last 4 bytes in the file header signifies the starting address of the BMP image data. The next DIB header represents the image information such as size, compression, color of the BMP image and the length of the header based on the version used for representing the image. The first 4 bytes are used for storing the DIB header size value. The next following fields are as follows 4 bytes for image width, 4 bytes for image height, 2 bytes for number of planes, 2 bytes for bits for pixel, 4 bytes for compression, 4 bytes for image size, 4 bytes for horizontal pixels per meter, 4 bytes for vertical pixel per meter and 4 bytes for colors used. The color palette typically depends on the number of colors mentioned in the DIB header. The Gap1 and Gap2 are usually optional for the BMP image. Depends on the image size described in the DIB header, the image data is presented in the pixel array header. The International color consortium (ICC) color profile header only exist when the Bitmap version 5 header is used as a DIB header to link the color profile data to the image. Due to the historical changes of the BMP headers, an adversary can leverage the different versions and can still be able to hide the content so that the BMP image can be used as a stegomalware carrier.

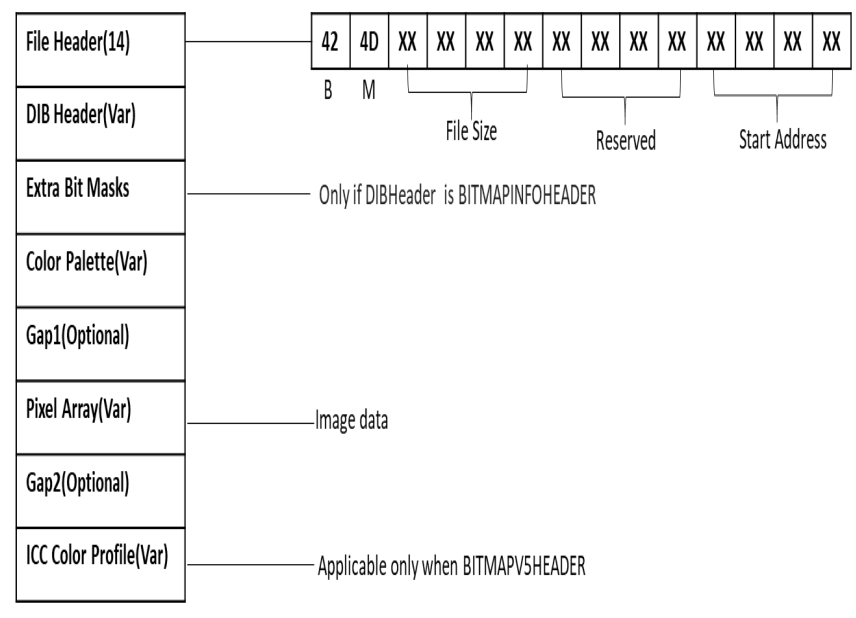

Fig. 5. BMP file structure format

We have also seen that the audio format WAVE is used as a malware stager in the advanced persistent threat attack life cycle. So, we are discussing the wave file structure format for understanding and analyzing the possible hidden areas in the audio file. Microsoft Resource Interchange File Format (RIFF) specification is commonly used for storing and managing the multimedia applications, which include image, audio, and video files. Some of the file formats that RIFF support are AVI, WAV, RDI, ANI, BND and RMI. The RIFF specification starts with chunk ID "RIFF" and the chunk size 4 bytes to represent the size of the chunk [71]. The Wave file may contain one chunk with RIFF specification. The wave chunk may further consist of two sub chunks. Those are "fmt" and "data". The format header represents the file format that the RIFF specification of the file referring, and, in this case, it is "wave" with hex value "0x57 0x41 0x56 0x45". The sub chunk 1 representing the audio format consists of the fields such as sub chunk 1 file size, audio format, number of channels, sample rate, byte rate and block align with each field varying the byte sizes from 2 to 4 . The audio format by default can be pulse code modulation (PCM) with the number of channels can be either mono (1) or stereo (2). The sample rate, byte rate and bits per sample can be selected based on the audio requirements. The audio data is stored in the sub chunk 2 . The data include the right channel and left channel samples representing the audio data. The data may be embedded with 
malicious payload to hide the stegomalware and use audio format WAV as a carrier to deliver it to the victim device.

\begin{tabular}{|c|c|c|c|c|}
\hline \multirow[b]{2}{*}{ Chunk ID (4) } & $R$ & I & $\mathrm{F}$ & $\mathrm{F}$ \\
\hline & 52 & 49 & 46 & 46 \\
\hline Chunk Size (4) & \multicolumn{2}{|l|}{ w } & V & $\mathrm{E}$ \\
\hline Format (4) & 57 & 41 & 56 & 45 \\
\hline Subchunk1 ID (4) & 66 & $6 d$ & 74 & 20 \\
\hline Subchunk1 Size (4) & $f$ & $\mathrm{~m}$ & $\mathrm{t}$ & \\
\hline Audio format (2) & & & & \\
\hline Number of Channels (2) & & & & \\
\hline Sample Rate (4) & & & & \\
\hline Byte Rate (4) & & & & \\
\hline Block Align (2) & & & & \\
\hline Bits Per Sample (2) & $d$ & $\mathbf{a}$ & $\mathrm{t}$ & $a$ \\
\hline Subchunk2 ID (4) & 64 & 61 & 74 & 61 \\
\hline Subchunk2 Size (4) & & & & \\
\hline Data (Var) & & & & \\
\hline
\end{tabular}

Fig. 6. WAV file structure format

Lessons Learned: The stegomalware used file formats JPEG, BMP, PNG, GIF, WAV are suitable for hiding the data or malware payload because these file format specifications poses new avenues for hiding the data. The files also contain unused space, which is used for storing the intended malware payload or other content. The file metadata also can be considered for hiding the data. Overall, it is evident that the mentioned file formats are perfect cover medium image file formats for hiding the malware. The proposal of universal stego detection solution for detecting the hidden malware in any of these file formats seems to be challenging.

\section{STEGANOGRAPHY}

Steganography process involves the cover medium, secret data and the technique/algorithm used to conceal the secret data in the cover medium. The proper selection of the steganography algorithm is needed to effectively hide the content in the cover medium and defend the steganalysis attacks. As the computational, processing capabilities on data increased over the period of time, advanced techniques are being proposed in the prior art to improve the maximum embedded content capabilities and resisting the steganalysis methods. In this section, various steganography algorithms are listed and discussed based on the chronological advancement of technology used for embedding the data in the cover image. The cover image steganography is considered for review because the images are extensively used for hiding the malware in the reported attacks historically as described in section IV. academic research contributions to image steganography are enormous, and image steganography is still considered as a very active research area. Additionally, the image steganography techniques are classified further based on algorithms applied on the spatial or transform domains with machine learning techniques. Furthermore, the GAN based for data hiding in cover images are also discussed along with their performances.

\section{A. Domain feature based Image Steganography}

The image steganography techniques are mainly categorized into spatial domain and transform domain techniques. In spatial domain, the steganography algorithm/technique is applied directly on the pixel values of the cover image to embed the intended secret data. For instance, in the LSB method, the least bit of every pixel in the image is leveraged to perform the Exclusive "OR" operation with secret data so that the least bit values of the pixels store the secret data. There are various spatial domain steganography techniques such as LSB, PVD, histogram shifting, difference expansion and multiple bit planes-based technique are proposed in the literature [29]. These methods include utilizing the gray images and color images as cover medium for data concealing purpose. As the steganographic operations performed directly on the pixel intensity values in the spatial domain for concealing the content, the decoding process may be easier with the existing tools and may detect the hidden content in the cover image. On the other hand, the transform domain converts the cover images into frequency or wavelet domains for data compression, fine detail separation in the data or localization purpose. In frequency domain, the DFT and DCT are well known and effective methods for image transform representation. The algorithms like DWT, IWT, CWT, and DTCWT are some of the most familiar in wavelet domain [29]. The transformed domain coefficients like DCT may be manipulated to hide the data and represent the images back in spatial domain to maintain the same quality. So, the transform domain based techniques can be more effective in steganography applications because decoding is complex process and steganalysis can be harder. Additionally, the operations like compression, scaling performed on the images may not have any impact on the quality of the images and hidden data. However, the transform domain techniques may require more computation operations and processing capacity than spatial domain techniques. Some of the secured steganography algorithms like HUGO [72], WOW [12], HILL [11] and UNIWARD [10] have also been proposed in the literature to effectively embed the data in spatial domain by adding the distortion and resist the steganalysis attacks.

There are number of works proposed or improved the existing works in the image steganography (pre GAN models) for the last two decades [29]. Unlike the existing studies performed detailed classification of image steganography algorithms [7], we have performed the review for the most widely used and well known image steganography algorithms and the compiled list is illustrated in the Table $\mathrm{V}$ The algorithms are arranged in chronological order for easy comparison and track the progress of the technological advancements. The proposed algorithm, their advantage and the generic comments are also included in the table $\mathrm{V}$ for each contribution. However, we have not provided further more details on minor contributions and additional relevant works [7] due to the limited space and align with the aim of our work.

Westfeld et al. [73] proposed JPEG domain based F5 steganography algorithm to address the issues present in the prior techniques such as JSteg, F3, and F4 around the year 
2001. In general, trade-off exist between the maximum embedding capacity and the resistance to defend the steganalysis attacks. Most of the algorithms JSteg, F3, and F4 are vulnerable to visual and statistical attacks when the embedding capacity is fixed. In order to improve the data embedding capacity and resisting against the statistical and visual attacks, the authors proposed steganographic algorithm "F5" in [73] considers two techniques such as permutative straddling and matrix encoding for effectively concealing the data in the image. In permutative straddling, F5 shuffles the DCT coefficients using permutations and then embeds the data in the permutation sequence. The matrix encoding helps to reduce the number of necessary changes needed in the cover image.

In [74], the authors proposed perturbed quantization based steganography to hide the data in frequency domain. Quantization is one of the essential step need to be performed in data compression or down sampling. In perturbed quantization technique, the data is embedded in the cover image while performing the image compression using standard JPEG domain techniques like DCT. When the pixel values are quantized to assign them as level based final integers, the data is embedded in the quantized process to adjust the final integers. The authors reported that their technique performed better than existing solutions in the prior art. Cancelli et al. [75] proposed spatial domain based MpSteg algorithm for hiding the data in images. MPSteg performs steganography based on the redundant basis image decomposition and matching pursuit. Although the PSNR value for the MpSteg is high, the MpSteg steganography is less detectable in comparison with LSB \pm embedded algorithm when applied sophisticated steganoalysis techniques. In 2007, Fredrich et al. [76] evaluated the JPEG steganography techniques to determine the maximum embed capacity techniques while securing against the steganalysis detectors. They deduce that PQt can embed maximum data compared to other techniques in JPEG domain and recommended syndrome coding methods to decrease the impact of embedding further. Kim et al. [77] proposed an efficient steganographic algorithm for hiding the data in LSBs of JPEG coefficients. To select the minimal embedding distortion coefficients, modified matrix encoding scheme is applied on the image in JPEG domain. The distortion results produced in the paper matches with theoretical distribution to embed the data in cover image.

In the year 2010, Pevny et al. [72] proposed HUGO algorithm to perform the steganography and is still considered as one of the benchmark algorithm for assessing the steganography and steganalysis solutions. HUGO uses high dimensional images for performing undetectable steganography. The distortion function as the weighted difference of the extended features from the steganoalysis is given as the steganography input to perform HUGO steganography such that to minimize the distortion. The feature set may contain more than $10^{7}$ features for HUGO, which may help reduce the security weaknesses. The HUGO algorithm was able to embed 7 times the message length compared to the LSB techniques for offering the same security level. The authors in [78] demonstrated the edge adaptive based steganography to select the data hiding positions in the image. The LSB is used to hide the content in the pixels and the sharp edges in the images are considered as image positions for hiding the content. This may help to protect the smooth regions with data corruption and protect from visibility and statistical attacks. In [12], the authors proposed WOW steganography algorithm to apply in wavelet domain. The algorithm uses syndrome trellis codes to minimize the expected distortion for a given payload. The embedded changes are done on the highly textured or noisy regions and avoid the sharp edges in an image, in contrast to the embedding done in edge adaptive algorithm. The WOW method is shown to be resistant to rich model based steganalaysis. The authors in [79] introduced a normalized perturbed quantization to select the DCT coefficients for embedding the data. The perturbation error, quantization step (QS), the modified magnitude of quantized DCT coefficient are considered for choosing the DCT coefficients. Additionally, the authors showed that normalized perturbed quantization improved security of the embedded data against steganalysis techniques.

Guo et al. [80] described UED algorithm to perform the uniform embedding along with syndrome trellis coding (STC). The uniform embedding distortion metric is measured based on the magnitude of the DCT coefficients and their intra and inter-block neighborhood coefficients. Uniform embedding makes less detectable with steganalysis techniques. The authors reported that UED obtained superior performance to defend against the steganalysis. In [10] presented universal distortion design method UNIWARD, which can be applied for arbitrary domains. The authors showed UNIWARD outperformed the existing solutions in spatial, JPEG and sideinformed JPEG domains. The embedded distortion in UNIWARD is calculated as the sum of the relative changes of coefficients in a directional filter bank decomposition of the cover image. The three algorithms such as S-UNIWARD, J-UNIWARD, and SI-UNIWARD are named based on the operating domains such as spatial, JPEG and side informed JPEG. Li et al. [11] defined a new cost function for minimizing the embedding distortion in steganography algorithm using high pass filter and two low pass filters. The HILL method locates the less predictable parts with high pass filter and then uses two low pass filters for clustering the low cost values. For the prior art steganalysis methods, Hill performed better than standard steganography algorithms such as HUGO, WOW and S-UNIWARD to hide the data. [81] synchronizes the embedded region in the cover image to improve the security against the steganalysis detection. A non-additive distortion function formed after cost assignment to each pixel enables the adjacent embedded changes to synchronize. The tests performed on HILL reveals that Synch-HILL exhibits more probability of error compared to the HILL when applied rich model steganalysis method.

Sedighi et al. [82] used the locally-estimated multivariate Gaussian cover image model to capture even non-stationary character of natural images and coined MiPOD steganography method, which minimizes the power of the optimal detector. The authors [83] improved the minimum distortion framework UERD by considering the mutual correlations among DCT blocks such that the less statistical detectability is achieved. 
The reported results dictate that the IUERD performed better than the UERD by a considerable margin and achieved comparable performable with J-UNIWARD. [84] proposed a joint distortion in JPEG using BBC principle. The BBC restrain the blocking artifacts caused by the inter block adjacent modifications to preserve the spatial continuity at the block level. The BBC addition into the Decomposing Joint Distortion (Dejoin) results in improving the large payload embedding capacity compared to the modern JPEG steganalyzers. [85] propose a fusion method to combine the HUGO, WOW, SUNIWARD steganography techniques. Additionally, the object detection method in the image is also used to select the complex textual regions for embedding the data. The authors [86] extended MiPOD in to JPEG domain and named it "JMiPOD". The MiPOD minimizes the statistical detectability and obtained comparable performance with prior art. Additionally, the authors also addresses the problem of data embedding in the JPEG domain color images and mentioned that JPEP color image steganography is a potential research direction to pursue. Cogranne et al. [87] performed natural steganography in JPEG images captured at ISO sensitivity. Natural steganography is performed on the natural cover images obtained from the CMOS sensor with ISO sensitivity and add stego signal, which mimics the sensor photonic noise. So, the stego image may mimic the sensitivity of the cover image and hide the date. The authors showed that JPEG domain NS (J-Cov-NS) achieved high embed capacity and security when tested with DCTR and SRNet steganalysis.

Lessons Learned: There were number of works proposed towards image steganography techniques starting from simple LSB to recent natural steganography based J-CoV-NS. However, there are few techniques such as HILL, WOW, UNIWARD in different domains, UERD, MiPOD stood out to be more secured and benchmarks for testing or comparing the new steganography or steganalysis techniques. The steganography in JPEG domain color images and natural steganography based techniques are two active areas to be explored for advancing the image steganography techniques (not including DL and GAN) [86] [87]. We also conclude that there is no prior art testing the security of the proposed steganography techniques over the years in stegomalware image datasets.

\section{B. Deep Adversial based Image Steganography}

The advancement in the hardware computation capabilities in recent times helped the rapid innovation of solving complex problems using deep learning in many application areas. Deep generative models are one of the deep learning models, which are received major attention due to the recent advancements. The deep generative models learn different data distributions in an unsupervised manner. There are two types of deep generative models. Those are Variational Auto Encoders (VAE) and GAN. The generative models have some drawbacks need to addressed when initially researchers explored the deep generative models for solving the problems. In order to address the drawbacks in generative models, Goodfellow [88] proposed GAN architecture in 2014. GAN mainly has two components such as generator and discriminator. The generator can randomly generate the input data and also considers the feedback to incorporate the error data. The discriminator network discriminate between the generated image and the expected image for the feedback. The combination of these two components can be used to produce fake multimedia content and data hiding as well.

Encoder decoder architecture are initially known to be used for data compression. The encoder helps to represent the same input with fewer data points and the decoder try to reconstruct the original input accurately given the encode output as decode input. However, this architecture may not be fitted into the generative model due to the encoder output is not regularized. Variational autoencoders mainly contain the encoder and the decoder modules. The authors in [89] proposed variational autoencoders to alleviate the regulation problem in traditional encoder and decoder architectures. These modules resemble the Convolutional neural network (CNN) layers with modification to the penultimate layers. Both VAE and GAN can be used to generate the data such as the image, text. Later on, these architectures also been used for media steganography and creating fake content for fooling the people.

The deep adversial model solutions proposed for image steganography are illustrated in the Table $\mathrm{VI}$ and discussed in chronological order.

Denis et al. [90] proposed a SGAN network to generate the steganographic images. SGAN contains a generator $G$ to produce the realistic images from noise, discriminator $\mathrm{D}$ to evaluate the produced image is real or synthetic and discriminator $\mathrm{S}$ performs the steganalysis to determine the given image is stego image or not. Haichao et al. [91] proposed SSGAN to generate secure cover image. The SSGAN uses WGAN [92] to generate the cover image in generator network, discriminator $\mathrm{D}$ to evaluate the visual quality of the generated images and GNCNN to perform the steganalysis in discriminator network S. The authors in [93] introduced CycleGan to perform image steganography in specific hiding one image in another image. The CycleGAN output nearly imperceptible, high frequency signal, which makes it is difficult to perform steganalysis. However, a careful design of the CycleGAN framework is needed to hide the data and achieve secure performance against steganalysis techniques. Tang et al. [94] introduced ASDL GAN framework to hide the secret data in images. The ASDL framework consist of a generator and discriminator networks. The generator network contains CNN layers with groups from 1 to 25 and the discriminator adapts the Xu's deep learning model [95] for steganalysis. The groups 2 to 24 configurations are the same in the generator network. Additionally, new activation function Ternary embedded simulator (TES) is proposed to generate the stego images according to the generator provided pixel level change probabilities.

Volkhonskiy et al. [96] proposed SEGAN model to hide the data in images using GAN. The SEGAN model contains one generative network, one discriminative network, and one decryption network. Considering the classic alice, bob and eva encryption problem mapping, the generative network performs the alice role to generate steganographic image, the decryption network performs bobs role to extract the hidden message in the image and the discriminator to determine the given 
TABLE V

SPATIAL, TRANSFORM DOMAIN IMAGE STEGANOGRAPHY TECHNIQUES

\begin{tabular}{|c|c|c|c|c|c|}
\hline Authors & Year & Algorithm/Technique & Domain & Advantages & Comment \\
\hline Westfeld et al. 731 & 2001 & F5 & JPEG & defend visual and statistical attacks & used permutative straddling and matrix encoding \\
\hline Fridrich et al. 74$]$ & 2005 & $\mathrm{PQ}$ & JPEG & $\mathrm{PQ}$ is the most secured technique compared to prior art till 2005 & the data is hidden in the Quantization fields \\
\hline Cancelli et al. 75$]$ & 2006 & MPsteg & Spatial & MPSteg less detectable than $\mathrm{LSB} \pm 1$ embedding & Embedding data in lines, corners,and flat regions \\
\hline Fridrich et al. 76 & 2007 & $\mathrm{PQt}$ & JPEG & large embedding capacity compared to state of the art till 2007 & syndrome coding methods could minimize the embedding impact \\
\hline Kim et al. 77$]$ & 2007 & MME & JPEG & The actual embedded distortion closely resembles the theoretical prediction & Embeds message in the LSB of JPEG coefficients \\
\hline Pevny et al. 72$]$ & 2010 & HUGO & Spatial & \begin{tabular}{|l} 
can hide 7 times longer messages compared to LSB for same security \\
\end{tabular} & minimizes the distortion caused by the embedding \\
\hline siva et al. 781 & 2010 & EA & Spatial & preserve the statistical and visual features in cover images & Edge regions are used to hide the content. \\
\hline Holub et al. [12] & 2012 & WOW & Wavelet & WOW outperforms HUGO for large embedding payloads & The embedded changes are done in noisy or highly textured regions \\
\hline Guo et al. 801 & 2012 & UED & jpeg & less detectability using existed steganalysis techniques & Syndrome trellis coding is used during the data embedding. \\
\hline Huang et al. 79] & 2012 & NPQ & JPEEG & Improved the Security performance of steganography algorithm & NPQ focuses on the selection of the optimal DCT coefficients for data hiding \\
\hline Holub et al. 10$]$ & 2014 & UNIWA & Arbitrary & UNIWARD outperformed state-of-art algorithms & the universal design of the distortion function can work in any domain \\
\hline Li et al. $[11]$ & $\frac{2014}{2014}$ & HILL & Spatial & better performance than HUGO, WOW, and S-UNIWARD & High pass filter and two Low pass filters are used in HILL \\
\hline Denemark et al. 81$]$ & 2015 & Synch-Hill & Spatial & $\begin{array}{l}\text { resists against the rich media steganalysis models } \\
\end{array}$ & non-additive distortion function to force adjacent embedding change to synchronize \\
\hline Sedighi et al. 821 & 2016 & MiPOD & Spatial & MiPOD provided comparable security to WOW, S-UNIWARD, HUGO & minimizes the power of the optimal detector \\
\hline Pan et al. 83$]$ & 2016 & IUERD & JPEG & Embedding capacity improved com & The mutual correlations among DCT blocks in JPEG a \\
\hline Li et al. $[84]$ & 2018 & IUERD- UpDist-Dejoin2 & Spatial and JPEG & The BBC improves the prior art JF & Preserves spatial continuity at block boundaries \\
\hline Meng et al. 85 & 2018 & fusion of 72$],[12][10]$ & Spatial & The fusion of WOW. HILL and UNIWARD obtained better performance & data is hidden in selected objection detection block region. \\
\hline Cogranne et al. 871 & 2020 & J-Cov-NS & JPEG & Achieved higher capacity and security when tested against SRNet & The security may decrease with non-linear developments \\
\hline Taburet et al. 86 & 2020 & J-MiPOD & JPEG & J-MiPOD showed competitive performance compared to prior art algori & first step towards embedding data into JPEG color images \\
\hline
\end{tabular}

image is real or generated image. In 2017, [97] proposed encoder decoder based architecture HayesGAN for embedding the data in the images. The technique involve alice, bob and eve as the game participants, whereas alice and bob acts as an encoder and decoder and the eve acts as a steganalyzer. Like any deep learning solution, the domain knowledge is not required to perform the image steganography using these models. [98] also proposed an encoder and decoder based deep network technique Hidden to hide the data in cover images. Additionally, an adversary network is used to discriminate the cover and stego image. An adversial loss is measured to correct the stego image generation with generator for each iteration until secured image is created by encoder. In the paper [99], the authors proposed GRDH scheme for image steganography. The generator network is used to generate the cover images; CycleGAN is applied to translate the image to image and then performed the mapping of noise to hiding data for concealing the data in images. The recovery process includes the same components used for image generation.

Zhang et al. [100] proposed StegnoGAN to hide the binary data in images. Three different models such as basic, residual and dense encoder architectures has been proposed to generate images. The decoder reconstruct the hidden data from the steganography image and another component "critic" provide feedback on the performance of the encoder to improve the secured stego generated image. Additionally, Reed-Solomon bits-per-pixel (RS- BPP) is proposed to evaluate the capacity of embedded data when using GAN models for steganography. Zhang et al.[101] demonstrated an ISGAN model to hide the grey scale image in the color image $\mathrm{Y}$ channel values. The architecture contains an encoder to embed the gray scale image in the color image and a decoder to reconstruct the original data from the stego image and a steganalyser tries to distinguish stego images from cover images to improve the overall security performance. All 3 networks in the ISGAN model contain CNN based layers. The layers may include the inception module in encoder, spatial pyramid pooling (SPP) blocks in steganalyser. [102] proposed ACGANs, in which the secret data transmission is done without modifying the cover images. A set of labels and the image database is considered for selecting the cover image. The intended sending message is mapped to the labels using dictionary in the sender end and use the label to pick the image from the database for data transmission. At the receiver side, the image is mapped with the corresponding label already stored in the database. The message related to the label mentioned in the dictionary is used for retrieving the message.

Zhangjie et al. [103] proposed a GAN based model HIGAN to hide the images in another image. The color image is hidden in another color image using HIGAN model. The HiGAN is composed of three subnetworks to perform the GAN based steganography. The encoder can hide the color secret image into a cover color image with the same size. The output of the encoder is fed into the decoder to extract the secret image. A discriminator is used to distinguish whether the input image contains secret image or not. The encoder contains 3 down sampling layers, 9 residual layers, and 3 up sampling layers to hide the image in another image. Wang et al. [8] proposed GAN model, which consists of U-NET based generator and the Xu model discriminator for performing the steganalysis of the images. The generator is reconstructed by combining the multiple feature maps and the reconstructed generator is used for proper information embedding.

The performance evaluation of the GAN based stego images are illustrated in Table VII. The Table VII categorized as the adversial model, performances, metric used, dataset used for models comparison. The SGAN generated stego images in [90] are applied to HUGO steganography analyzers for assessing the security performance. The detection accuracy of the SGAN generated images reduced by $30 \%$ in comparison with \pm 1 embedding algorithm when HUGO steganalyser used. The authors [91] compared the performance of the SSGAN with the SGAN [90]. We can clearly see that the time to run 7 epochs took 227.5 seconds for SSGAN, whereas SGAN completed the epochs in 240.3 seconds. Additionally, the steganalyser detection accuracy for SSGAN 0.72 is much lower than the SGAN accuracy 0.90 for the GAN generated images. The authors in [94] showed that the ADSLGAN performed better than S-UNIWARD in terms of security performance. For $0.1 \mathrm{bpp}$ embedding rate, ASDLGAN achieved $26.92 \%$, whereas SUNIWARD obtained $42.53 \%$ accuracy when applied the Xu's steganalysis model. [96] presented data encryption based GAN model SEGAN has obtained the quality of encryption/decryption 99.98 and 99.96 for the MNIST and CIFAR-10 when the percentage of reconstruction bits is 16 . Furthermore, the paper also demonstrated that SGAN and DCGAN models 
TABLE VI

DeEP AdVERsial BASEd IMAGE STEGANOGRAPHy TECHNIQUeS

\begin{tabular}{|l|l|l|l|l|l|}
\hline Authors & Year & Architecture & Networks & Advantage & Comment \\
\hline Denis et al. [90] & 2017 & SGAN & 3 & The SGAN model reduced the steganalyzer detection accuracy & DCGAN [104] model also performed well to secure the cover images. \\
\hline Haichao et al. $[91]$ & 2017 & SSGAN & 3 & SSGAN performed better than SGAN & Visual quality is better and relaistic \\
\hline Chu2017 et al. 93$]$ & 2017 & CycleGAN & 4 & Image hiding in another image & CycleGAN adversial attacks vulnerability helps to hide information \\
\hline Tang et al. [94] & 2017 & ASDL-GAN & 2 & The automatic learning of the embedding change probabilites at the pixel level & The proposed model is only evaluated for spatial domain \\
\hline Denis et al. [96] & 2017 & SEGAN & 3 & Secure and adaptive steganographic image generation & Symmetric key is used for encryption prior to hiding \\
\hline Hayes2017 et al $[97]$ & 2017 & HayesGAN & 3 & First encoder and decoder based adversial learning for steganography & The secured performance still has room for improvement \\
\hline Zhu et al. $[98]$ & 2018 & Hidden & 3 & Secured performance better than HUGO, S-UNIWARD, WOW & The secured performance can still be improved. \\
\hline Zhang et al. 99$]$ & 2019 & GRDH, CycleGAN & 4 & First data hiding without cover image modification & Embedded Capacity is limited compared to traditional methods \\
\hline Zhang et al. [100] & 2019 & SteganoGAN & 3 & SteganoGAN can accomdate higher payloads while evading the detection & Three different models are proposed to generate steganoGAN image \\
\hline Zhang et al. [101] & 2019 & ISGAN & 3 & Can hide the gray scale image in the color image & Model robustness need to be improved \\
\hline Zhuo et al. [102] & 2018 & Stego-ACGAN & 3 & Cover image modification is not required for data hiding & Only set of messages stored in dictionary can be embedded. \\
\hline Zhangjie et al. 103$]$ & 2020 & HIGAN & 3 & Higher visual quality and stronger security & Encoder network uses upsample, downsample and residual layer \\
\hline Wang et al. [8] & 2020 & GAN with feature maps & 2 & Better distortion measurement and secured steganographic scheme & Reconstructed U-NET for Generator and Xu model for Discriminator \\
\hline
\end{tabular}

decrease the detection accuracy to an extent of similar to random classifier, which is impressive. Hayes2017 et al. [97] tested the performance of the HayesGAN on the datasets Boss, Celeba. Even though HayesGAN is not performed better than the state of the art steganalysis techniques like WOW, HUGO, the model showed that the image steganography can be performed using HayesGAN. For Boss datasets, HayesGAN achieved $79 \%$ accuracy whereas for celeba datasets, it obtained 90\% accuracy. The Hidden model proposed in [98] obtained $50 \%$ detection rate for the COCO dataset. The performance achieved by Hidden model is better than the HUGO, WOW and S-UNIWARD. However, the comparison of the Hidden with other GAN models is not available. The GRDH scheme proposed in [99] able to perform well to securely hide the data in the images. On the dataset Celeba, the PSNR value 22.665 is obtained when random noise is given as an input for image generation case model BEGAN. But, the embedded capacity of the proposed method is very limited. The authors [100] proposed SteganoGAN for embedding data in the cover images is tested on two datasets Div2 K, COCO and the performance metrics such as accuracy, Reed Solomon Bits Per Pixel, Peak signal-to-noise ratio are used for comparison. SteganoGAN performed better to securely hide the data. The payload capacity in Div2K dataset rather than the COCO dataset when performing the steganalysis

The ISGAN model [101] performance on three different datasets LFW, PASCAL-VOC12 and ImageNet shows that the CNN based steganalysis network able to detect $78.5 \%, 74.3 \%$ and $73.6 \%$ images accurately. Furthermore, the authors showed that ISGAN achieved the state-of-the-art steganography performance in terms of the structure similarity index (SSIM) and PSNR. The author's proposed model in [102] achieved 100 percent prediction accuracy of auxiliary classifier when the training step is 10 . The HiGAN model [103] performance on the Imagenet2012 showed that the structural similarity between stego image and original images is $94 \%$ and the peak signal-to-noise ratio is 30.95 . These performance results indicate that HiGAN did performed well to hide the color image on another color image. The performance of the U-NET based Generator and Yu's model Discriminator [8] on BOSSBase dataset shows that error rate $34.80 \%$ is obtained when the spatial rich model with ensemble classifier used. The authors reported that their proposed model achieved better performed than S-UNIWARD, ASDL-GAN.

Lesson Learned: The deep adversial based steganographic algorithms has potential to generate maximum embedding capacity and less detectable stego images. Overall, based on the performance analysis of the state-of-the-art GAN based solutions, it is difficult to compare deep adversial stego model using a unique approach because the researchers use different datasets, dataset count, steganalyzer, performance metrics for evaluation. However, we can conclude that GAN based steganography solutions performed well compared to the traditional steganography solutions such as WOW, HUGO, UNIWARD in terms of embedding capacity, secured data hiding. We also found that there are no existing deep adversial stego solutions focused on hiding the malware in images. It is interesting to generate stegomalware using deep adversial solutions and evaluate the performance of those solutions to evade the advanced next generation solutions.

\section{STEganalysis}

Steganalysis is the act of determining if the cover medium is hidden data or not and identifying the steganographic algorithm; estimating the hidden data if the cover medium is hiding the data and extracting the hidden data from the stego medium. Steganalysis can be performed in many ways. Signature based steganalysis is the simplest way and straight forward process. Steganalyzer may take the advantage of the steganography tool signature when embed the data to confirm if the given digital medium is stego or cover medium. For instance, "hiderman" steganography tool add the letters "CDN" at the end of the cover file when embedding the data. So, extracting the bytes of the file and comparing with known signature or specific patterns maybe helpful to identify the stego file. The file can be an image, audio, video or other format. We discuss the "file" herein in the context of images, since we determined that stegomalware is leveraging the cover image for hiding seen in section IV Later on, the statistical properties of the images in different domains are considered as a feature vector to capture the stego content and ML techniques are applied to classify stego and cover images. The spatial and JPEG rich models along with ensemble classifiers shown good performances in state-of-the-art ML category but they are not optimal models. The detailed review and categorization of the various feature extraction and classification using ML for steganalysis is described here [9]. Recently, the deep learning based steganalysis received major attention and reported best stego detection performances compared to the rich model based detection techniques [32]. In this section, our aim is to discuss and categorize the best state-of-art steganalysis techniques existed for the last two decades in chronological 
TABLE VII

DEEP AdVERSIAL BASED BASED IMAGE STEGANOGRAPHY PERFORMANCE

\begin{tabular}{|l|l|l|l|l|l|}
\hline Authors & Year & Architecture & Dataset & Performance & Metrics \\
\hline Denis et al. [90] & 2017 & SGAN & Celebrities & Accuracy reduction 0.624 to 0.499 \\
\hline Haichao et al. [91] & 2017 & SSGAN & CelebA & Epochs:7, run time: 227.5 s, Accuracy: 0.90 to 0.72 & Run time, Accuracy \\
\hline Chu2017 et al. [93] & 2017 & CycleGAN & - & - & - \\
\hline Tang et al. [94] & 2017 & ASDL-GAN & BossBase & 0.1 bpp, Accuracy: 26.92\% \\
\hline Denis et al. [96] & 2017 & SEGAN & MNIST, CIFAR10 & MNIST: 99.98, CIFAR10: 99.96 \\
\hline Hayes2017 et al [97] & 2017 & HayesGAN & Boss, Celeba & Boss:79\%, Celeba:90\% \\
\hline Zhu et al. [98] & 2018 & Hidden & COCO & $0.203 b p p$, bit error $<10^{-5}$, detection rate: 50\% \\
\hline Zhang et al. [99] & 2019 & GRDH & CelebA & BEGAN, PSNR:22.665, StyleGAN, PSNR:28.333 \\
\hline Zhang et al. [100] & 2019 & SteganoGAN & Div2K, COCO & Depth=5,COCO Accuracy:0.84, RS-BPP:3.43 PSNR:29.73 & Accuracy, RS-BPP, PSNR \\
\hline Zhang et al. [101] & 2019 & ISGAN & LFW, PASCAL,ImageNet & LFW: 0.785 PASCAL-VOC12: 0.743, ImageNet:0.736 & Detection Accuracy \\
\hline Zhuo et al. [102] & 2018 & Stego-ACGAN & MNIST & Prediction accuracy:100\% at training step 10 \\
\hline Zhangjie et al. [103] & 2020 & HIGAN & ImageNet2012 & SSIM:0.94, PSNR:30.95 \\
\hline Wang et al. [8] & 2020 & U-NET & BOSSBase & $0.20 b p p$, Error rate: 34.80 \\
\hline
\end{tabular}

order highlighting the research progress and also investigate if the existing steganalysis methods are used for stegomalware detection.

\section{A. Image domain based feature and rich models steganalysis solutions}

Johnson et al. [105] discussed different ways to perform the steganalysis on various stego tools such as Hide4PGP, Mandelsteg, Syscop, hideandseek, Stools for uncovering the hidden data. Although there were no experiments designed in the paper, it opened up new ideas for approaching the steganalysis. The authors in [106] proposed QMF based high order statistics feature extraction method and then applied SVM on the extracted features for steganalysis. The higher order statistics could resist counter based attacks. Lyu et al. [106] considered image quality measures (IQM) for capturing image features and applied multivariate regression to discriminate between cover and stego images. The IQM features are obtained using ANOVA technique. The authors in [107] performed feature extraction in JPEG domain using DCT coefficients, which are first order and second order statistics and then applied linear classifier for the steganalysis of the images. The steganographic algorithms Outguess, F5 and Model based steganography with and without deblocking are tested for evaluating the proposed method. Also, the co-occurrence matrix of DCT coefficients is identified as one of the essential feature for JPEG image steganalysis. Kenneth et al. [108] described the markov chain based interpixel dependencies as a feature sets for detecting the stego images, where the data is hidden using spread spectrum. The ML technique SVM is employed on the extracted features to classify the images. However, the proposed inter pixel dependencies based model is shown to be applicable only spread spectrum based steganography image detection. The authors [109] proposed BSM based features for detecting the stego images. SVM is considered for classifying the images. The correlation between the bit planes and the binary texture characteristics within the places can be different for cover and stego image to distinguish them. Zou et al. [110] used two-dimensional markov model of thresholded prediction-error of an image for stego detection. The transition matrix of markov along the chain in vertical, horizontal and diagonal direction is considered as a feature and applied to the SVM for classifying the images. The state-of-the -art steganographic techniques spread spectrum(SS), non-blind SS, Quantization index modulation (QIM), LSB are considered for testing the proposed model. Jan et al. [111] used different steganalysis features such as SPAM, markov process, cartesian calibration doubles (CC-PEV), cross-domain Feature (CDF) to detect the YASS steganographic images. The SVM with a Gaussian kernel is used as image classifier by applying the four feature sets on different YASS settings from 1 to 12 . The combined feature set CDF achieved good performance to classify the images compared to the three individual feature sets.

The low dimensional feature selection along with linear classifier or SVM tend to be finding difficulty to classify content adaptive stego algorithms like HUGO proposed in 2010. So, researchers considered high dimensional feature extraction by performing the low feature selection from multiple submodels also called base learners. Then, ensemble classifiers are used for selecting the optimal base learners and obtaining the better detection performances. The recent works in this direction are discussed now onwards. Fridrich et al. [112] proposed higher-order local model estimators of steganographic changes (HOLMES) to consider the combination of the MINMAX residual and the co-occurrence matrices with markov chain features to maximize the detection capabilities of the content adaptive algorithms like HUGO. Additionally, ensemble classifier with voting method for fusion is considered to classify the images. Fridrich et al. [113] also presented the spatial rich models and ensemble classifiers for stego detection. Multiple residual classes such as first, second, third order, edge and square are described for feature (neighboring pixels cooccurrence matrices) generation of the submodel. In ensemble classifier, the feature selection of the submodels is done using different classes like CLASS-q, ITERATIVEBEST-q, BEST-q-CLASS, BEST-q etc. The out of box (OOB) error can be used as a parameter to select the best performed classes for ensemble classification. The author's work prompted a new research direction towards using rich model and ensemble classifiers for steganalysis.

Kodovsky et al. [114] performed rich model and ensemble classifier stego detection in JPEG domain and proposed cartesian-calibrated JRM (CC-JRM). A detailed investigation of individual subsets performance is performed on the 6 well known JPEG steganographic algorithms. Adding Cartesian 
calibration increases the number of features and also improved the performance of steganalysis. Furthermore, the paper determined that BCHOpt is the most secure, and MBS and YASS are by far less secure stenographic algorithms when tested on the union of CC-JRM and the SRM. The detailed comparison of the various other feature sets on the JPEG steganographic algorithms is discussed in the paper [114]. Holub et al. [115] proposed PSRM to retrieve the statistically significant features from the residual samples rather than the cooccurrence matrices representation of residual samples in SRM. In PSRM, the neighboring residual samples are projected onto a random space and the first order statistics of the projections are considered as a feature set. The authors showed that the PSRM can be applied in multiple domains with few changes and tested against various secured steganographic algorithms for detection. Holub et al. [116] also proposed low complexity feature set generation method DCTR to use in JPEG domain. These features are obtained from the decompressed JPEG using 64 kernel DCT. The authors [117] presented PHARM feature set for stego detection in JPEG images. In contrast to the PSRM, PHARM only uses few number of residual from support kernels and represent them in first order statistics of their random projections. The computation cost and number of dimensions needed are much lower than the JRM and PSRM models.

Song et al. [118] proposed 2 dimensional gabor filters to capture the image texture and edges from different scales and orientations and the histogram features are extracted from the filtered images so that adaptive steganographic images can be detected accurately than the state-of-the-art models. The gabor filter is applied on the JPEG decompressed images. The extracted feature set is applied to the ensemble classifier to classify the images. The experimental results shown that gabor filter performed better than the state-of-the-art feature set techniques like CC-JRM and DCTR. The authors in [119] proposed SGF for generating new features and then adding to the color rich model features to enhance the feature set. The Gaussian filter features can identify the minor changes done to the image during the embedding process. These new features enhance the detection capability against the three stego algorithms S-UNIWARD, WOW, and Synch-HILL. Xia et al. [120] improved the GFR [118] by proposing the symmetric merging of different Gabor filters and weighted histograms by considering the position of residuals. The combination of the symmetric merging and weighted histograms along with GFR is defined as GFR-Gabor symmetric merging and weighted histograms(GFR-GW) and the symmetric merging with GFR is denoted as GFR-Gabor Symmetric Merging (GFR-GSM). The inclusion of these two methods improved the detection performance against the J-UNIWARD and UED. The authors in [121] improved the PHARM for enhancing the stego detection capabilities. Three changes have been done on PHARM to improve the performance. The authors reduce the maximum projection matrix size, selection of more than one phase pair per projection and considering the transposition symmetry to improve the PHARM performance. Additionally, the proposed improved PHARM detection accuracy better than DCTR feature sets. Xia et al.[122] proposed improvements to DCTR and GFR feature sets for efficient stego detection. The different residual images, multiple filter sizes and different symmetrization rules by considering the filter type, filter size are utilized to improve the performance. The improvement models DCTRD, DCTR-W and SCA-DCTR, GFRD, GFR$\mathrm{W}$ and SCA-GFR are presented in [122]. The detection performance improved for SCA-DCTR compared to DCTR$\mathrm{W}$ and the performance of DCTR-W improved compared to DCTRD. Similarly, the GFR improved version also follow the same trend. However, the feature extraction time increases for SCA-DCTR compared to DCTR-W and also increases DCTRW compared to DCTRD. Overall, the detection performances increased for improved versions of DCTR and GFR. Fend et al. [123] presented maximum diversity cascade filter residual (MD-CFR) feature set for steganalysis of images. The cascade filters are formed by combining the base filters and maximum diversity is considered for cascade filter selection. These filters are convolved with JPEG decompressed images to obtain the maximum diversity cascade filter residuals. The steganalysis is performed on four steganographic algorithms to test the proposed feature set with the state-of-the-art feature sets.

Lessons Learned: Image steganalysis has advanced over the last decades with initially research progress focused on feature and ML based detections followed by rich model and ensemble classifier based solutions. Some of the steganalysis methods like CDF, SRM, JRM, PSRM, DCTR, PHARM, GFR, SGF stand out to be effective detection solutions in the pre deep learning era. However, we believe that the conventional image steganalysis research seems to be slow down with more contributions towards improving the state-ofthe-art solutions GFR, DCTR, PHARM roughly for the last five years. The reason could be the availability of the deep learning technology with computation capabilities in the last few years and rapid growth of using deep learning in various application include steganalysis. The fact that deep learning models could provide good performance results compared to the conventional steganalysis is another reason towards this shift. Interestingly, we have not found any works evaluating the performance of the existing techniques to detect the stegomalware hidden in images or proposing new steganalysis techniques for stegomalware detection.

\section{B. Image domain based feature and rich models steganalysis performance}

The higher order statistic and SVM based method in [106 achieved 98.5\% classification accuracy for the stego images generated by the jsteg tools, when the natural images are considered for the evaluation. The authors [124] performance evaluation on ANOVA based feature set steganalysis achieved in the best case $85 \%$ detection rate for PGS technique. The steganalysis performance in [124] still had a room for improvement. [107] evaluated the blind feature based steganalysis on algorithms F5, Outguess, Model based (MB1) and Model based with deblocking $(\mathrm{Mb} 2)$. The results presented in the paper show that the model based techniques MB1 and MB2 performed well with low detection reliability values 0.16 , 0.21 . Further, the Outguess based stego images are almost 
TABLE VIII

IMAGE DOMAIN BASED FEATURE AND RICH MODELS STEGANALYSIS SOLUTION

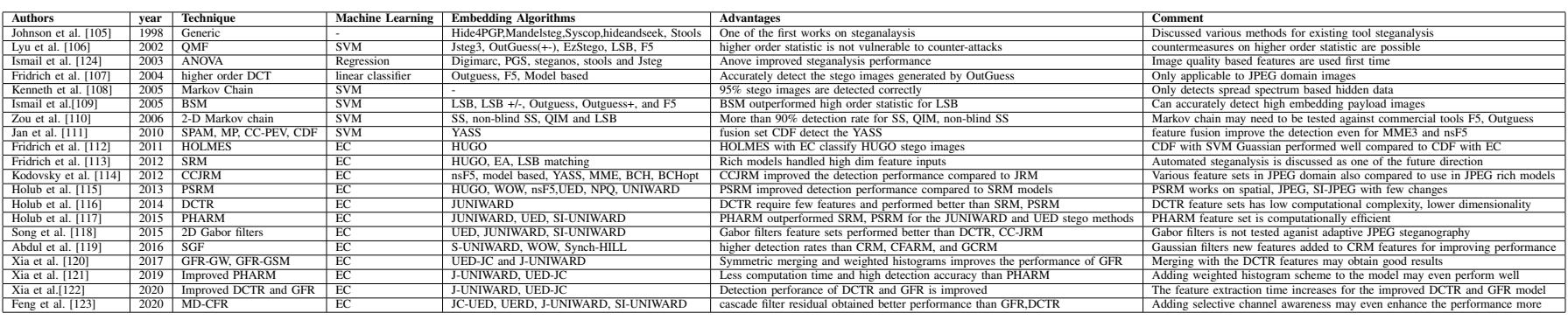

detected using the blind features with linear classifier when the bpnzac is set to 0.05. Kenneth et al. [108] evaluated their Markov chain and SVM based detection model in both the locally adaptive and globally adaptive hiding cases with spread spectrum method. The results showed that globally adaptive hiding performed well to classify the images compared to the locally adaptive hiding when considered the image datasets from diverse sources. The BSM performance evaluation in [109] on stego algorithms Outguess, F5 and LSB \pm showed that the accuracy increased as the embedding payload bpp increases. When the bpp is 15 , the proposed method obtained $92.17 \%$ for $\mathrm{LSB} \pm$ detection. The Outguess and F5 could not accommodate $15 \mathrm{bpp}$ for hiding the data in images. Zou et al. [110] obtained good performance results for stego image classification for four stego algorithms. When the experiments are performed with non-linear kernel, their model obtained around $90 \%$ accuracy for SS, non-blind SS, QIM and around $85 \%$ accuracy for the LSB steganography image datasets. The article also showed that the non-linear kernel SVM performed better than linear kernel in stego image classification.

The authors [111] tested the performance of different feature sets on YASS algorithm and showed that the best performed CDF detected YASS with $P_{e}<15 \%$ when bpnzac is 0.003 . Additionally, for the MME3 and nsF5, the CDF obtained < $10 \% P_{e}$ when bpnzac is 0.003 . This shows that feature fusion methods help to achieve good performance even in steganalysis. The performance evaluation of the HOLMES in [112] showed that the HOLMES methodology for stego detection is effective even for secure algorithm HUGO. For embedding capacity of $0.5 \mathrm{bpp}$, the detection error for HOLMES is $12 \%$ for HUGO compared to $7.3 \%$ with $\mathrm{LSB} \pm$ embedding. This shows that HUGO is much more secured than \pm embedding for HOLMES detection. HOLMES also performed well compared to CDF [111] when tested on HUGO. The SRM with EC proposed in [113] is evaluated on Bossbase datasets. The top39 submodels with 12,753 dimensional are selected for the evaluation of SRM+EC on LSB matching, HUGO, EA algorithms. For embedding payload bpp is 0.05 , the SRM+EC is outperformed the Gaussian-SVM in terms of detection error and running time. The detection error for TOP39 rich model is 0.42 for HUGO model, 0.3255 for EA and 0.274 for LSB matching. The authors concluded that HUGO is secured in comparison to the LSB matching and EA. Overall, the rich models with EC are proved to be an efficient future direction to detect HUGO algorithms. The proposed model CC-JRM in [114] obtained detection error 0.422 for $\mathrm{BCH}$ and 0.448 for $\mathrm{BCHOpt}$ when the embedding payload is 0.1 . On the other hand, the JPEG techniques nsF5, MBS and MME obtained detection error 0.3298, 0.037 and 0.4307 respectively. This clearly shows that MBS is the least secured and BCHOpt is the highest secure algorithms out of the 6 JPEG steganography algorithms for CC-JRM. In [115], the PSRM performance is evaluated on Bossbase and Leticia datasets. As shown in the Table VIII, the PSRM performance for the secure algorithms in each domain is presented for comparison. UNIWARD clearly performed better than other algorithm in each domain. When the embedded payload is 0.1 , S-UNIWARD achieved $P_{e} 0.3564$ in spatial domain, which is higher than HUGO, WOW. The PSRM also showed more resilience for J-UNIWARD and SI-UNIWARD detection. The feature set DCTR performance in JPEG domain is evaluated in the paper [116]. The authors mentioned that DCTR feature sets had low OOB error compared to the PSRM, SRM methods for the dataset BossBase when tested on J-UNIWARD algorithm, which means DCTR can detect hidden data better than classic SRM, PSRM.

Holub et al. [117] proposed PHARM feature set performed better than the JRM, PSRM, DCTR for the J-UNIWARD, UED algorithms. For the quality factor 75 and embedding payload 0.1 in JPEG, The PHARM obtained detection error 0.31 for the J-UNIWARD and 0.18 for the UED models, which are better performances than JRM, PSRM. Abdul et al. [119] proposed Gaussian filters feature sets to enhance the stego detection. For the embedded payload bpp 0.1, the detection rate for S-UNIWARD, WOW and Sync-HILL is $70.16 \%, 69.09 \%$ and $70.54 \%$ respectively, which is reported to be better than color rich models. Song et al. [118] evaluated the detection performance of the GFR feature set on UED, J-UNIWARD, SI- UNIWARD detection. When the Quality factor is set to 75 and embedded payload is 0.2 , the detection errors for UED, J-UNIWARD and SI-UNIWARD are 0.18, 0.3 and 0.47 respectively. Furthermore, the detection error for GFR is lower than the CC-JRM and DCTR, which is good. Xia et al. [120] evaluated the performance of the improved GFR version GFR-GW, GFR-GSM on the UED and J-UNIWARD algorithms. The improved GFR version is better performed on UED compared to the J-UNIWARD. Additionally, the improved version GFR-GW(0.2943) performed better than GFR$\operatorname{GSM}(0.3071)$ for UED. The improved PHARM in [121] has obtained detection error 0.2911 for UED-JC and 0.4023 for J- 
UNIWARD algorithms when the selected parameters were QF 75 and bpnzac is 0.1. Improved DCTR and GFR performance in [122] shows that detection error for $\operatorname{DCTRD}(0.2859)$ and DCTRD-W(0.2789) for UED is improved compared to JUNIWARD detection. The detailed performance description of the 6 variations of improved DCTR, GFR feature sets is given in detail [122] and it is clear that improved versions significantly improved the detection performance. For $0.1 \mathrm{bpn}$ zac and QF 75, MD-CFR [123] performed better for JC-UED with detection error 0.277 in comparison to $\operatorname{UERD}(0.373) \mathrm{J}$ UNIWARD(0.412) and SI-UNIWARD(0.494) detection.

Lessons Learned: The steganalysis solution performances are evaluated and compared with other solutions using detection error and detection accuracy metrics. The researchers also used the standard stego algorithms such as HUGO, UNIWARD, HILL, WOW, UERD to assess the effectiveness of steganalysis solutions. The steganalysis solutions mentioned in chronological order in Table IX showed that detection performances improved year by year. The notable steganalysis best performed techniques are CDF in early 2009 followed by the Rich models around 2011 and then PHARM, GFR, DCTR since 2015. But, none of these works evaluated performance on stegomalware detection and it is interesting to see how effective these methods to detect the hidden malware in cover images.

\section{Deep learning models for Image steganalysis}

Even though neural networks proved to process the high dimensional data and reduce to one dimension [125], the neural network struggle to achieve optimal learning time and thought to be less effective than machine learning algorithms until 2010 [126]. But, the advancements in hardware GPU capabilities and innate feature learning capabilities has made deep learning a first choice to addressing the complex classification problems in different applications.

Determining the feature sets for image steganalysis required domain knowledge and deep understanding of the image pixel level operations. Additionally, the performance need to be improved for the accurate stego image detection using the combination of feature sets and machine learning EC methods. Furthermore, the average running time for high dimensional feature images using ML classifiers is higher. So, researchers explored the application of deep learning models in steganalysis. CNN is a well known to be used for image processing and classification in deep learning. The modification of CNN in accordance with classifying the hidden data images and cover images may be helpful for obtaining optimal detection performance in steganalysis. We discuss various deep learning models present in the prior art addressing the image steganalysis and Table Xillustrates the different deep learning steganalysis solutions proposed in the prior art. The research works are listed in chronological order for ease of solution comparison and research progress analysis.

Qian et al. in [127] proposed GNCNN steganalysis model for stego image detection. The GNCNN architecture contains an image processing layer, five convolutional layers and three fully connected layers. The uniqueness of the GNCNN is that the Gaussian function is considered as non-linear activation function instead of the Relu to add at the output of the convolution layers. The authors described that Gaussian function is better to distinguish the stego and cover image. The GNCNN is tested against the WOW, HUGO and SUNIWARD steganography algorithms and also compared with the SRM and SPAM feature sets with SVM classifier. Xu et al. 95] presented a CNN based steganalysis architecture $\mathrm{Xu}$-net to detect the residual based stego detection. The architecture contains a high pass filter for generating residual from the image, the convolution module and the linear classification module. The convolution module comprises 5 groups of convolution blocks including the convolution layers, average pooling, activation function like Relu, TanH and batch normalization. The linear classification module contains fully connected and soft max for image classification. The novelty of the Xu-net is the design of the CNN layers for stego detection. The Xu-net is evaluated against the residual based algorithms S-UNIWARD and HILL and reported that Xu-net provides comparable detection performance with SRM feature sets. Ye et al. [128] proposed SCA-TLU-CNN architecture for image steganalysis. The SCA-TLU-CNN consist of the 9 convolutional layers, 1 fully connected layer and 1 softmax for binary classification. Each convolution layer includes the author's proposed activation function $T L U$ instead of ReLU. The selection channel awareness is incorporated in the first layer of the proposed model. The model is tested against WOW, S-UNIWARD and HILL and compared with SRM and $\operatorname{maxSRMd} 2$ feature sets.

Chen et al. [129] presented CNN based payload estimator for stego detectors. The CNN model softmax module is replaced with mean square error function and the payload values are considered as classification labels. The binary classifier output is fed into 3 fully connected layers with Relu activation function to obtain payload estimate. The estimators have been tested with spatial domain and JPEG domain stego algorithms. The authors [130] proposed modified CNN solution for detecting the J-UNIWARD stego images. The proposed CNN architecture includes a preprocessing layer to convert JPEG into spatial domain and then applying filter banks to retrieve the magnitude of DCT subbands followed by truncation to limit the input data. After the preprocessing, the data is processed through 20 convolution layers while pooling is achieved with convolution with stride 2 rather than max or average pool. The linear classification contains a fully connected layer followed by a softmax layer for assigning the class labels to the feature vectors. Inspired by Xunet [95], the authors [131] proposed phase aware CNN architecture for JPEG phase awareness detection. The phase split layer is introduced at the output of Group 2 feature maps in Xunet to subsample each feature map into 64 sublattices. Based on how the phases processed later in phase aware CNN, two architectures such as Pnet and Vnet are proposed. In Pnet architecture, the 64 phases are channeled independently, and the net becomes wider towards the last layers. This model requires more memory and the computation complexity increases. On the other hand, all the 64 channels are concatenated into a group and fully 
TABLE IX

IMAGE DOMAIN BASED FEATURE AND RICH MODELS STEGANALYSIS PERFORMANCE

\begin{tabular}{|c|c|c|c|c|c|}
\hline Authors & Year & Technique & Dataset & Performance & metrics \\
\hline Johnson et al. 105] & 1998 & Generic & & & \\
\hline Lyu et al. 106$]$ & 2002 & QMF & Natural images & jsteg: $98.5 \%$ & classification accuracy \\
\hline Ismail et al. 1241 & 2003 & ANOVA & fapp2 & PGS:85\% Stools:75\% Jsteg:70\% & Detection rate \\
\hline Fridrich et al. [107] & 2004 & higher order DCT & Greenspun & 0.05bpnzac, F5:0.24 Outguess:0.87 MB1:0.21 MB2:0.16 & Detection reliability \\
\hline Kenneth et al. 108 & 2005 & markov chain & Diverse sources & Spatial: Local adaptive: $0.985,0.893$, globally adaptive: $0.982,0974$ & Recall, Precision \\
\hline Ismail et al. $[109]$ & 2005 & BSM & greenspun & 15bpp: LSB $\pm: 91.06$ & Detection accuracy \\
\hline Zou et al. [110] & 2006 & 2-D markov chain & Multiple sources & 0.1bpp, SS:89.15\% non-blind SS:94.10\% QIM:97.03\% LSB:86.30\% & Accuracy \\
\hline Jan et al. [111] & 2010 & SPAM, MP, CC-PEV, CDF & mother image $\mathrm{db}$ & $0.003 \%$ bpnzac, $\mathrm{CDF}: P_{e}<15 \%$ & probability of error \\
\hline Fridrich et al. 112] & 2011 & HOLMES & BossBase & 0.5bpp, HUGO, Holmes: $12 \%$ CDF:28.4\%; LSB \pm , Holmes:7.3\% CDF: $13.4 \%$ & Detection error \\
\hline Fridrich et al. 113] & 2012 & SRM & BossBase & 0.05bpp, HUGO: TOP39: 0.424; EA: TOP39: 0.3255; LSB: TOP39: 0.274 & Running time, Detection error \\
\hline Kodovsky et al. 1141 & 2011 & CC-JRM & CAMERA db & 0.05bpnzac,nsF5:0.3298,MBS:0.0373,MME: $0.4307 ; 0.1 \mathrm{bpnzac}, \mathrm{BCH}: 0.422, \mathrm{BCHopt}: 0.448 ; 0.077 \mathrm{bpnzac}, \mathrm{YASS}: 0.303$ & detection error \\
\hline Holub et al. $[115]$ & 2013 & PSRM & BossBase, Leica & 0.1 bpp, S-UNIWARD:0.3564; QF75, 0.1 bpnzac, J-UNIWARD: $0.4319 ;$ QF75,0.1bpnzAC, SI-UNIWARD:0.4952 & Detection error \\
\hline Holub et al. 116] & 2014 & DCTR & BoSSBase & DCTR:0.1523, SRM:0.2127, PSRM:0.148 & Outof the box(OOB) error \\
\hline Holub et al. 117 & 2015 & PHARM & BOSSbase & QF75,0.2bnpzac, J-UNIWARD:0.31; UED: 0.18; SI-UNIWARD:0.47 & Detection error \\
\hline Song et al. 118$]$ & 2015 & 2D Gabor filters & BossBase & QF75, 0.2 bpnzac UED:0.18, J-UNIWARD:0.3, SI-UNIWARD 0.47 & Detection error \\
\hline Abdul et al. 119$]$ & 2016 & SGF & BossBase & 0.1bpp, S-UNIWARD:70.16\% WOW:69.09\% Sync-HILL:70.54\% & Detection rate \\
\hline Xia et al. 1201 & 2017 & GFR-GW, GFR-GSM & BossBase & 0.1bpnzac, QF75, J-UNIWARD: GFR-GSM:0.4058 GFR-GW:0.3994; UED-JC: GFR-GSM: 0.3071 GFR-GW:0.2943 & Detection error \\
\hline Xia et al. 121$]$ & 2019 & Improved PHARM & BossBase & 0.1bpnzac QF75, J-UNIWARD:0.4023, UED 0.2911 & detection error \\
\hline Xia et al. 122$]$ & 2020 & Improved DCTR and GFR & BossBase & 0.1bpnzac QF75, J-UNIWARD: DCTRD:0.4120, DCTRD-W:0.4082; UED: DCTRD:0.2859, DCTRD-W:0.2789 & detection error \\
\hline Fend et al. 123 & 2020 & MD-CFR & BossBase & QF75, 0.1bpnzac, J-UED:0.277, UERD:0.373, J-UNIWARD:0.412, SI-UNIWARD:0.494 & detection error \\
\hline
\end{tabular}

convolved into the convolution layer of the next group in Vnet architecture. The Vnet run faster and takes advantage of the correlation between different phases in the subsequent layers of the architecture. Yedroudj et al. [132] presented Yedroudj-net CNN architecture for spatial steganalysis. the authors designed the Yedroudj-net by considering the best layer components, modules used in ye-net, Xu-net for stego classification. The Yedroudj-net comprises a preprocessing layer, five convolution layers similar to Xu-net and typical CNN classification module. The preprocessing layer contains predefined high pass filter for learning the robust signals in images. The convolution layer also followed by the batch normalization, Relu non-linear activation function, absolute value activation in convolution block 1 , average pooling with stride 2 and global average pooling. The classification module contains three fully connected layer followed by softmax activation function for image classification. The proposed model is tested against WOW and S-UNIWARD spatial algorithms.

The authors in [133] designed a training model ReSTNet concatenating the prior art [95] $\mathrm{Xu}-\mathrm{CNN}$ architectures while choosing different activation functions. The ReST-Net consists of three parallel subnets and concatenated with the classification module to perform the image classification. Each subnet is the modified $\mathrm{Xu}-\mathrm{CNN}$ architecture with the convolution group 2 and 4 are replaced with dynamic activation modules (DAM). The DAM comprises three parallel convolution layers, in which one of the ReLU, Sigmoid and $\mathrm{TanH}$ activation function is applied to each convolution layer to learn the steganography artifacts. The concatenated feature maps are passed through the next group. Average pooling, batch normalization and activation functions are used in all the layers of the convolution groups and the last layer contain global average pooling. The classification module includes the fully connected layer and softmax function for classification. Tsang et al. proposed [134] CNN steganalysis architecture adapted from Ye-net [128] for stego detection of any image size. The batch normalization is added to each Relu of the Ye-net. Additionally, the 9th convolution stride changed to 1. The moments extraction module is added to capture moments such as maximum, minimum, average and variance of feature maps to identify the image size and other characteristics. For a larger image, the network with moments is trained with smaller size crop images first. Then, the larger image is used to extract the moments and trained two Inner product layers on the larger image moments to obtain larger image detector. The authors in [135] presented deep residual architecture universal steganalyser "SR-net" for spatial and JPEG stego detection while minimizing heuristics and externally enforced elements in the model. The SR-net is composed of 12 convolution layers and the layers can be any one of the defined 4 layers types. The 4 layer types are defined based on the existence of residual shortcuts and pooling. The two layers of type 1 don't contain the residual shortcuts or pooling. type 2 of layer 2 to 7 contain residual shortscuts and no pooling. The layers 8 to 11 has type 3 , in which both pooling and residual shortcuts exist. type 4 has one last convolution layer in the SR-net, which contain average pooling layer and no residual shortcuts.

Deng et al. [136] proposed global covariance pooling based $\mathrm{CNN}$ steganalysis architecture to improve the training time and also improve the detection performance compared to the stateof-the art SR-net architecture. The proposed model comprises the preprocessing layer with HPF and truncation, 4 groups containing the convolution layers and the linear classifier for image classification. The novel global covariance pooling layer is incorporated in the Group 4, which already contain 2 convolution layers. The group 1 includes 4 convolution layers followed by average pooling with stride 2 . The group 2 and 3 contains 2 convolution layers followed by average pooling with stride 2 . The model is tested against the SRnet, the proposed model with average pooling instead of Global covariance pooling. The authors in [137] presented OneHot CNN architecture to effectively detect the stego images in JPEG domain. The OneHot encodes the DCT coefficients of the images into binary volumetric representation of the DCT plane. The encoded DCT values are fed to two convolution modules followed by Global average pooling layer. The classification module consists of fully connected layer for binary image classification. The proposed model is tested against the nsF5 and J-UNIWARD detection and showed that OneHot CNN is better than JRM. Li et al. [138] performed steganalysis based on the feature fusion of SRNet base learners and used ensemble classifiers to obtain the better performance. Several decision combinations such as majority voting, product combination are used to combine the SRNet base learners. Furthermore, the ensemble classifiers are applied to the base learner's serial and parallel feature fusion to obtain the results. 
The authors in [139] proposed CNN based architecture Zhunet for efficient detection of image steganography. Various changes in preprocessing, convolution layers and pooling layer has been proposed to model the Zhu-net. The $3 \times 3$ kerenl sizes are recommended instead of $5 \times 5$ in the preprocessing layer to reduce the number of parameters and capture the local region features. Additionally, the depth wise convolution layer is proposed to improve the signal-to-noise ratio, utilizing the channel correlation of the residual in the convolution modules. Finally, the spatial pyramid pooling (SPP) is also presented as the last pooling layer prior to the classification module to represent the features with multi level pooling. Further, additional datasets are considered to further boost the detection performance. The authors in [140] performed data enrichment by improving the datasets so that the detection performance increases. The data enrichment method "pixelsoff" removes few pixels from the image to enrich the datasets. As the number of images in the dataset increases, it helps to increase the overall detection performance. The pixels-off is tested against the Yedroudj-net and covariance pooling CNN for performance evaluation.

The authors in [141] presented LSER network to improve the detection performance. The LSER architecture contains $3 \times 3$ convolution layer followed by local resource group layer and two local source downsample group layer. Then, second order global pooling with iterative matrix square root normalization, fully connected layer and softmax to classify the stego and cover images. LSER mainly contains two characteristics such as ensemble residual and local source skip connection. The residual block with no batch normalization is considered in the LSER architecture. Local source skip connection allows bypassing features from different levels so that precise features are used for representation. The LSER is tested against against both spatial and JPEG algorithms. Jang et al. [142] proposed feature aggregation networks (FANet) and leveraged Relu6 as an activation function for image stego detection. The feature aggregation module contains two down sampling, two up sampling and one residual block to aggregate the feature maps at various level and resolutions. The feature aggregation is performed near the input data to expand the number of channels of convolutions blocks. Overall, the FANet architecture contains 16 blocks varying the block types from 1 to 7 followed by linear classifier for classification. The authors in [143] presented Reparameterization Vgg (Repvgg) [144] block and Squeeze-and-excitation (SE) based feature fusion steganalysis architecture SFRNet. The Repvgg block contains a stack of $3 \times 3$ convolution layers and Relu activation, while having multibranch topology. It will improve the inference and efficient memory utilization. The Squeeze-and-excitation block is used to improve the detection accuracy rate. The SFRNet architecture comprises the feature extraction and fusion layer followed by the Repvgg blocks with $T L U, \operatorname{Re} L U$ and average pooling spreading over five stages and three SE blocks are incorporated between the stages. The linear classifier includes three fully connected layer and softmax function for stego and cover image classification.

The DFSE-Net proposed in [145] composed of the diverse filter module (DFM) and squeeze-and-excitation (SE) modules for effective detection against the content adaptive steganographic methods. The diverse filter modules combine three different scale convolution filters to obtain the diverse information and squeeze-and-excitation module to strengthen the key channels. The DFSE architecture contain preprocessing layer with $T L U$ and High pass filter, convolution with batch normalization, three DFSE modules including DFM and SE and then followed conventional linear classifier with fully connected and softmax function. The authors in [15] presented GBRASNet architecture, which comprises the preprocessing layer with filter banks, multiple depth wise and separable convolution layers for improving the performance and skip connections to speed up the learning. The authors in [16] proposed deep fractal network based architecture SFNet for image steganalysis. The SFNet comprises multiple CABR(Convolution, Absolute, Batch, RelU) and CBR(Convolution, Batch, RelU blocks with balanced width and depth in the network followed by the global averaging, fully connected and softmax function.The SFNet does not require preprocessing steps and can achieve good performance using depth and wide coverage of the network. Mondal et al. [146] presented H-Stegonet hybrid deep learning technique by combining the MHSRMNet and StegoRUNet. StegoRUNet is the modified version of Residual U-net [147]. MHSRMNet process the feature vector into multiple bins to reduce the dimension and then concatenate those bins to get the final decision output. The two nets are combined and used dense and dropout layers to perform the classification. The authors tested the model against HUGO and WOW S-UNIWARD detection.

Lessons learned: The image steganalysis research has shifted towards deep learning steganalysis than the conventional steganalysis is lately. There are number of deep learning steganalysis solutions proposed in the literature to address stego detection. The solutions mainly focus on proposing changes in preprocessing layer, activation function, convolution layers arrangement, blocks fusion, linear classifier to capture the stego elements. As seen in conventional steganalysis review, the stego algorithms WOW, HILL, UNIWARD, UED are mainly used for performance comparison. As the deep learning field is actively progressing in image processing applications, there are more research opportunities in future to utilize the deep learning models for image steganalysis and improve the performances even more. We have not found any literature works performing stegomalware detection using deep learning models and pose to be a security malware research direction in future to test the existing deep learning models and proposing new models for stegomalware detection.

\section{Deep learning Steganlysis performance}

The performance evaluation of GNCNN [127] illustrated that GNCNN has obtained comparable performance with SRM feature sets and better performance compared with SPAM feature sets. When the payload capacity $0.3 \mathrm{bpp}$, GNCNN achieved detection error 0.338 on HUGO, 0.343 on WOW and 0.359 on S-UNIWARD stego detection. These results showed that S-UNIWARD is slightly more secured than HUGO, WOW for GNCNN steganalysis. The Xu-net [95] achieved 
TABLE $X$

DEEP LEARNING STEGANALYSIS

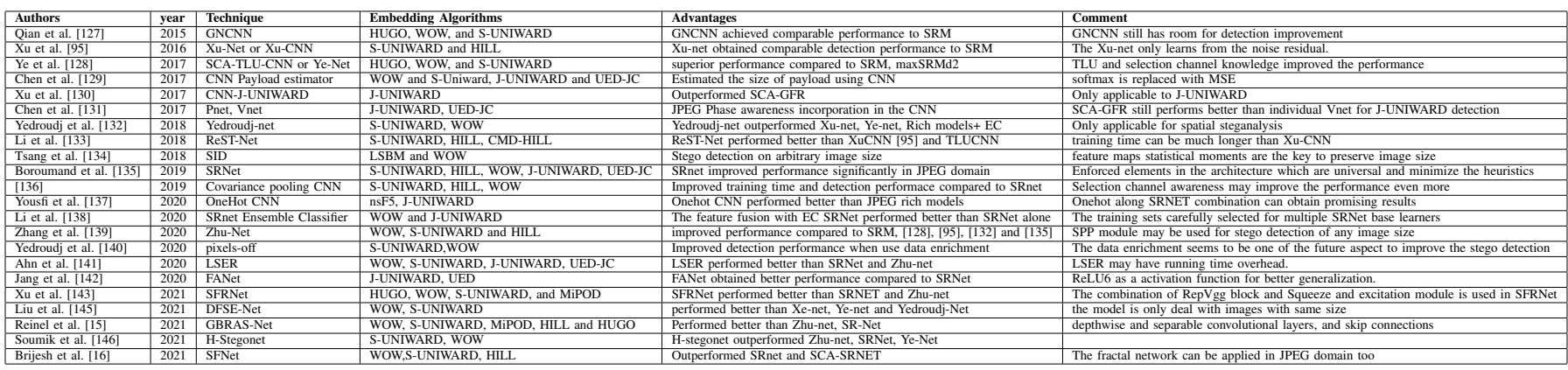

detection accuracy $80.24 \%$ for S-UNIWARD and $79.24 \%$ for HILL when the embedded capacity is selected as $0.4 \mathrm{bpp}$. $\mathrm{Xu}$-net architecture showed similar performances for both Hill and S-UNIWARD. Additionally, for S-UNIWARD, SRM obtained detection accuracy of $79.53 \%$, which is comparable performance to $\mathrm{Xu}$-net. Ye et al. [128] model SCA-TLUCNN performance evaluation depicts that the detection error for WOW, S-UNIWARD and HILL are 0.1691,0.2224 and 0.2538 respectively for resampled images when the payload is $0.2 \mathrm{bpp}$. HILL is more secure against SCA-TLU-CNN when compared to WOW and S-UNIWARD. But, we can clearly see that the SCA-TLU-CNN performed well compared to GNCNN and Xu-net to detect the stego images, although the dataset sample size used for evaluation may not be the same. In [129], the detector used for payload estimator has the following detector error when applied to spatial and JPEG algorithms. The detection error for WOW and S-UNIWARD are 0.2796 and 0.3452 , whereas the detection error for $\mathrm{J}$ UNIWARD and UED-JC is 0.4040 and 0.2450 when quality factor is 75. The authors in [130] tested the modified CNN for J-UNIWARD detection using BoSSBase and CLS-LOC image dataset. With the parameters QF75 and embedding capacity of 0.2 bpnzac, the modified CNN achieved 0.1947 detection error for J-UNIWARD detection and performed better than SCA-GFR. The phase aware CNN proposed in [131] performance shows that phase aware $\mathrm{CNN}$ can detect UED-JC better than J-UNIWARD with almost $50 \%$ reduction in detection error, as shown in Table $\mathrm{XI}$. Further, the Pnet, Vnet performed better than SCA-GFR detection in UEDJC. However, SCA-GFR still performed slightly better than Pnet and Vnet in J-UNIWARD detection. The authors [132] presented CNN based spatial steganalyzer "Yedroudj-net" for stego detection. The Yedroudj-net able to detect WOW better than S-UNIWARD stego images. For embedding rate $0.2 \mathrm{bpp}$, the $P_{e}$ for S-UNIWARD is $36.7 \%$, which is higher than the WOW $27.8 \%$. Additionally, the Yedroudj-net outperformed the prior art Xu-net(32.4\%), Ye-net(33.1\%) and SRM+EC(36.5\%) for the WOW detection. Similar performance for Yedroudj-net is achieved for J-UNIWARD detection when compared to the existing models. So far. we could see that SCA-TLU-CNN reported best performances compared to all the other models in all the image domains.

The ReST-Net model in [133] is evaluated for $\mathrm{S}$ UNIWARD, HILL and CMD-HILL detection. The model is efficiently detecting S-UNIWARD than the HILL and CMDHILL stego images. The detection accuracy for S-UNIWARD is 71.35, whereas the HILL and CMD-HILL produced 70.64 and 65.14 when embedding rate is $0.2 \mathrm{bpp}$. Additionally, the ReST-Net performed better than the Xu-CNN, TLU-CNN models for the three stego algorithms. This performance improvement is due to the feature maps concatenation from parallel subnets as well as layers in convolution groups. The size independent detector (SID) in [134] is tested against with RTRIP detector for proving that the SID works for any image size. For embedded rate $0.12 \mathrm{bpp}$ and the image size $256 \times 256$, the SID achieved detection error 0.259 on WOW, which is comparable to RTRIP detection error 0.261. The same trend followed for $1024 \times 1024$ image size with detection error 0.1391 and 0.1445 for the SID and RTRIP respectively. The SR-net architecture [135] performance is evaluated on spatial and JPEG stego detection to compare the performance against the state-of-the-art methods. For embedding rate $0.2 \mathrm{bpp}$, the detection error of the SRnet spatial models S-UNIWARD, HILL and WOW are $0.20,0.23$ and 0.16 respectively. This shows that SRnet can detect WOW slightly better than HILL and S-UNIWARD. Additionally, the SRNet is also performed better than the SCA-YeNet for all the three stego algorithms. In JPEG domain, the model is tested against the J-UNIWARD and UED-JC. With QF 75, 0.2bpp, the detector errors for JUNIWARD and UED-JC are 0.1889 and 0.568. It shows that J-UNIWARD is more secured against SRNet compared to the UED-JC. The model obtained superior performance in JPEG domain compared to the prior arts. At this point, SR-net and SCA-TLU-CNN models has given the best performances for deep learning based stego detection.

The global covariance layer based CNN [136] performance shows that the detection accuracy 80.05, 77.11 and 84.33 obtained for S-UNIWARD, HILL and WOW detection when the embedding rate $0.2 \mathrm{bpp}$. The WOW stego images are more likely to be detected with the covariance CNN in comparison with the S-UNIWARD and HILL. Under the same experimental setting, the SRnet obtained 84.05 detection accuracy and the model with average pooling obtained 83.78 detection accuracy for the WOW detection. This shows that the author's model slightly improved the detection performance compared to SR-net. Additionally, the average time taken to complete one iteration during training is significantly reduced for the covariance CNN (65ms) compared to the SRNet (261ms). 
The peformance of OneHot CNN in [137] showed that the detection error of OneHot CNN is 3.49 for nsF5 and 7.36 for J-UNIWARD, when the quality factor and embedding rate is set to 100 and $0.2 \mathrm{bpp}$. Furthermore, the authors mentioned that OneHot CNN performed better than JRM with detection error 4.17 for the same parameter settings. They also showed that combination of SRnet with OneHot CNN fusion improves the performance significantly compared to the SRnet for nsF5, J-UNIWARD, UED-JC. In [138], the authors evaluation of SRNet base learners serial and parallel feature fusion combinations with ensemble classifier showed that both feature fusion methods performed better than SRnet for WOW and J-UNIWARD detection. When the embedding rate 0.2 and serial feature fusion is used for classification, the $P_{e}$ is 0.1872 for WOW and 0.2367 for J-UNIWARD detection. The similar performance 0.1878 for WOW and 0.2308 for JUNIWARD is achieved when used parallel feature fusion and EC. For embedding rate $0.2 \mathrm{bpp}$, the Zhu-net performance in [139] obtained the detection error 0.233 and 0.285 for WOW and S-UNIWARD detection. The authors reported that zhu-net performed better than Xu-net, Ye-net, SRM+EC, Yedroudjnet, SRnet. Furthermore, the addition of the more datasets, the zhu-net detection error reduced from 0.233 to 0.131 , which is significant performance improvement. The testing performance of the "pixels-off" in [140] shows that YedroudjNet with 400 pixels off Boss dataset achieved 23.5 detection error for WOW and 26.5 for S-UNIWARD in comparison with 27.71 for WOW and 35.42 for S-UNIWARD for the original datasets. This clearly showed that "pixels off" data enrichment techniques improves the decision performance for YedroudjNet. The similar phenomenon is also true for covariance CNN. The LSER [141] performance is evaluated using BoSSBase and BOWS2 datasets. For embedding rate 0.2bpp, the LSER achieved 0.2375 and 0.2846 for WOW and S-UNIWARD detection in spatial domain. It shows that WOW is more secured than S-UNIWARD against the LSER detection. For embedding rate 0.2 bpnzac and QF 75, LSER obtained 0.1176 and 0.3115 detection error for UED-JC and J-UNIWARD detection in JPEG domain. Overall, the J-UNIWARD is more secure than other three algorithms for stego detection using LSER. Additionally, the authors mentioned that LSER performed well compared to SRNet and Zhu-net in both spatial and JPEG domain for all the four stego techniques.

The FANet performance in [142] is tested using ALASKAV2 image datasets. The FANet obtained 71.22 and 84.24 detection accuracy for J-UNIWARD and UED when the QF 75 and embedding rate $0.2 \mathrm{bpnzac}$ is selected. The J-UNIWARD stego images are more secured compared to the UED when FANet is used for detection. Further, the FANet performed better than the SRNet (70.14 and 79.32 for J-UNIWARD and UED) when the embedding rate is $0.2 \mathrm{bpnzac}$ and $\mathrm{QF} 75$. The performance of DFSE-NET [145] reveals that detection error for WOW and S-UNIWARD is 0.247 and 0.341 respectively when the embedding rate is chosen to be $0.2 \mathrm{bpp}$. The DFSE-NET is also performed better than Xu-Net, Ye-Net and YedroudjNet with detection errors $0.345,0.306$ and 0.332 respectively. The SFRNet proposed in [143] performance showed that MiPOD is slightly secured than other spatial techniques against the SFRNet steganalysis. Additionally, the article reported that SFRNet outperformed prior art models SRNet, Zhu-net, DFSE-Net in terms of detection accuracy and testing time. SFRNet took $9 \mathrm{sec}$ whereas other model consume more than $25 \mathrm{sec}$ to test the images. Mondal et al. [146] architecture HStegonet obtained the classification error 35.5 and 41.4 for the WoW and S-UNIWARD, when the embedding rate is $0.2 \mathrm{bpp}$. When the embedding rate $0.2 \mathrm{bpp}$, GBRAS-Net [15] obtained the best detection accuracy 80.3 for WOW and least detection accuracy 68.5 for HILL. In comparison with popular prior art solutions like Zhu-net (76.9), SR-net (75.5) and Ye-net (66.9) for WOW detection, GBRAS-Net performed much better. The SFNet [16] performance indicated that HILL is more secured compared to WOW and S-UNIWARD for stego detection, as shown in $\mathrm{XI}$, when the embedding rate is $0.2 \mathrm{bpp}$. Furthermore, the authors reported that SFNet performed better than SRNet and SCA-YeNet.

Lessons learned: The literature deep learning model performances are mostly compared using detection accuracy, detection error and BoSSBase is considered as a standard benchmark database for evaluation. Based on the performance evaluations of deep learning models listed in Table $X$, the models SCA-TLU-CNN, SR-Net, Zhu-net, LSER, SFR-Net and GBRAS-Net showed notable and improved detection performances. These models may be used as a reference for performance comparison in DL steganalysis future contributions. One of our future work is to evaluate the performances of the existing DL solutions in stegomalware conceal in images detection.

\section{Stegomalware Detection framework}

In this section, we describe the stegomalware creation process, multimedia malware analysis framework to identify the enterprise organization network targeting stegomalware, and the different network architectures in datacenter, cloud or multicloud environments used to deploy the malware analysis framework.

\section{A. Stegomalware Creation Process}

The stegomalware creation process involves selecting the cover or carrier medium like image, audio, or video files as an input file; the intended hiding data may be $\mathrm{C} \& \mathrm{C}$ server IP address, URL, malware payload, shellcode commands, other malicious intent Linux or windows commands to run on the compromised victim machine when triggered, and inputting the cover medium and hiding data to the chosen steganography tool or algorithm for generating the stegomalware file. The Figure 7 illustrates the top-down representation of the stegomalware creation process.

Steganography can be performed using the tools mentioned in Table IV] or GAN based stego generation or adversary customized tools like WOW, UNIWARD, GFR, HILL for generating more sophistication stegomalware to evade the victim security defense tools. There are different ways to hide the content in the cover medium so that the content embedded carrier file looks like the original carrier. The steganography tools may use the steganography algorithms like classical LSB, 
TABLE XI

DEEP LEARNING MODELS FOR IMAGE STEGANALYSIS PERFORMANCE

\begin{tabular}{|c|c|c|c|c|c|}
\hline Authors & year & \begin{tabular}{|l} 
Technique \\
.
\end{tabular} & dataset & Performance & metric \\
\hline Qian et al. $[127]$ & 2015 & GNCNN & BOSSbase 1.01, ImageNet & $0.3 \mathrm{bpp}, \mathrm{HUGO}: 0.338$, WOW: $0.343, \mathrm{~S}-\mathrm{UNIW}$ & Detection error \\
\hline Xu et al. 951 & 2016 & Xu-Net & BOSSbase 1.01 & 0.4bpp, S-UNIWARD:79.53 and HILL:75.47 & Accuracy \\
\hline Ye et al. 128$]$ & 2017 & SCA-TLU-CNN & BOSSbase 1.01, BOWS2 & $0.2 \mathrm{bpp}$, WOW: 0.1691, S-UNIWARD: 0.2224, HILL: 0.2538 & Detection error \\
\hline Chen et al. 129$]$ & 2017 & CNN Payload estimator & BossBase & $\alpha 0.1$ WOW:0.2796, S-UNIWARD:0.3452; QF75, JUNI75:0.4040, UED-JC75:0.2450 & Detection error \\
\hline Xu et al. 130 & 2017 & CNN based J-UNIWARD detection & Bossbase v1.01,CLS-LOC & QF-75, bpnzAC:0.2 CNN-J-UNIWARD: 0.1947 & Detection error \\
\hline Chen et al. 131$]$ & 2017 & Pnet, Vnet & BossBase & QF75, 0.2bpnzac, J-UNIWARD(Pnet: 23.50 Vnet:24.57); UED-JC(Pnet: 9.55 Vnet:10.07) & Detection error \\
\hline Yedroudj et al. 132 & 2018 & Yedroudj-net & BOSSBase v.1.01 & $0.2 \mathrm{bpp}$, WOW: $27.8 \% \mathrm{~S}$-UNIWARD: $36.7 \%$ & Probability error \\
\hline Li et al. 1331 & 2018 & ReST-Net & BOSSBase v1.01 & $0.2 \mathrm{bpp}, \mathrm{S}$-UNIWARD:71.35, HILL:70.64, CMD-HILL:65.14 & Detection accuracy \\
\hline Tsang et al. 134$]$ & 2018 & SID & BOSSbase 1.01 & 0.12bpp WOW; 0.01 change rate LSBM; 256x256 LSBM SID:0.243 WOW:0.259; $1024 \times 1024$ LSBM:0.0856 WOW:0.1390 & detection error \\
\hline Mehdi et al. $\mid 135]$ & 2019 & SRNet & BOSSbase and BOWS2 & $0.2 \mathrm{bpp}$, Spatial(S-UNI: 0.2090 , HILL: 0.2353 , WOW: 0.1676$) ;$ QF75, JPEG(J-UNIWARD-0.1889, UED-JC -.0568$)$ & Detection Error \\
\hline Deng et al. $[136]$ & 2019 & Covariance pooling based CNN & BOSSBase and BOWS2 & $0.2 \mathrm{bpp}, \mathrm{S}$-UNIWARD: 80.05, HILL:77.11, WOW:84.33 & Detection accuracy \\
\hline Yousfi et al. 137$]$ & 2020 & Onehot CNN & union of BOSSbase 1.01 and BOWS2 & QF:100, 0.2bpp nsF5: 3.49; 0.4bpp J-UNIWARD:7.36 & Detection error \\
\hline Li et al. $[1381$ & 2020 & SRNet ensemble classifier & BOSSBase v1.01 & SF-EC, 0.2 bpp WOW:0.1872, 0.2 bnnzac J-UNIWARD:0.2367; PF-EC, 0.2 bpp WOW:0.1878, 0.2 bpnzac J-UNIWARD:0.2308 & Prob error \\
\hline Zhang et al. 1391 & 2020 & Zhu-Net & BOSSBase v1.01, BOWS2 & $0.2 \mathrm{bpp}$. WOW: $0.233 \mathrm{~S}$-UNIWARD: 0.285 & detection error \\
\hline Yedroudj et al. 140$]$ & 2020 & pixels-off & BOSS, Alaska & 0.2bpp, Yedroudj-Net: WOW: 23.5, S-UNIWARD: 29.3; CovPool-Net: WOW: 23.34, S-UNIWARD: 26.64 & detection error \\
\hline Ahn et al. 141$]$ & 2020 & LSER & BOSSbase 1.01, BOWS2 & 0.2bpp, WOW:0.2375; QF75, 0.2bpnzac, S-UNIWARD:0.2846, UED-JC:0.1176, J-UNIWARD:0.3115 & detection error \\
\hline Jang et al. 142 & 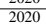 & $\begin{array}{ll}\text { LSLNet } \\
\text { FAN }\end{array}$ & 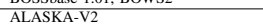 & $0.2 \mathrm{bpnzac}$, QF75, J-UNIWARD:71.22 UED:75.09 & Detection accuracy \\
\hline$\frac{\mathrm{XU} \text { et al. }[142][}{143]}$ & $\frac{2021}{2021}$ & \begin{tabular}{|l} 
SFRNet \\
SFRet
\end{tabular} & \begin{tabular}{|l|l} 
ALASKA-V \\
BOSSBase 1.01
\end{tabular} & 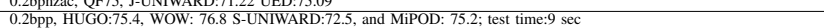 & $\begin{array}{l}\text { Detection accuracy } \\
\text { Detection test time }\end{array}$ \\
\hline Liu et al. 145 & 2021 & DFSE-Net & BosSBase & $0.2 \mathrm{bpp}$, WOW:0.247, S-UNIWARD:0.341 & Detection error \\
\hline Reinel et al. 15 & 2021 & GBRAS-Net & BoSSBase, BOWS & 0.2bpp, WOW: 80.3 S-UNIWARD:73.6, Mi-POD:68.3, HILL:68.5, HUGO:74.6 & Detection accuracy \\
\hline Mondal et al. 146$]$ & 2021 & H-Stegonet & BosSBase, BOWS & BoSSBase, $0.2 b p p$, WOW: 41.4, S-UNIWARD: 35.5 & classification error \\
\hline Singh et al. 16$]$ & 2021 & SFNet & BOsSBase & 0.2 bpp, WOW:0.1579, S-UNIWARD:0.1964, HILL: 0.2438 & Detection error \\
\hline
\end{tabular}

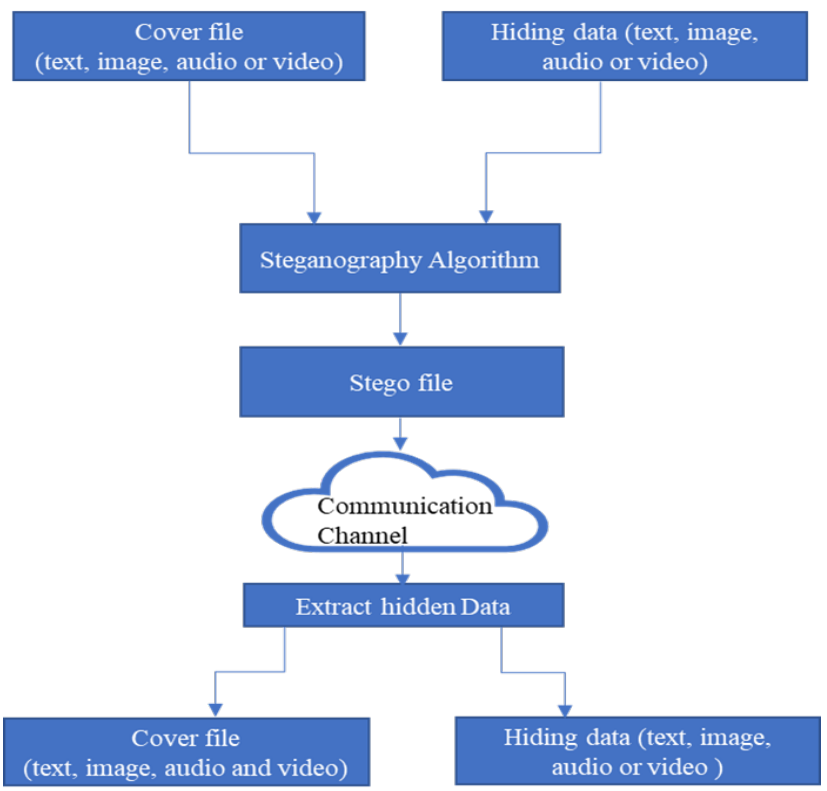

Fig. 7. Generic Stegomalware creation process

PVD or transform domain techniques like DCT, DWT to hide the malicious content. In addition, the hidden content is firstly encrypted with private key using symmetric encryption algorithms like RC4, DES or AES to have another layer of protection along with steganography. Consequently, the stegomalware is delivered to the victim machine through communication channel. The most generic communication channel in most of the stegomalware cases is internet. Subsequently, the receiver collects the stegomalware file through phishing emails or another form of weaknesses in the victim machine. An exploit kit running on the victim machine instruct the malicious code to extract the hidden content from the stego image. If the hidden content is the malicious attacker server IP address or URL, then the exploit kit uses those artifacts for connecting to the remote server without being caught by the security tools. Then, it may download another malicious code script from the remote server to perform the exfiltration of the confidential data or encryption keys to encrypt the victim data. It is also possible that the malicious code hidden in the stego malware and then decode the content to run on the victim machine. The victim can only see the cover file and may not be able to know the hidden malicious activity happening behind.

We have collected the stegomalware samples from virushare.com and performed the string analysis using " $\mathrm{xxd}$ " command in Ubuntu machine. The Figure 8 shows the excerpt of the stegomalware representing the HTML and JavaScript code in the cover image and their hexadecimal representation of the values. The basic string analysis tools like "strings", "exiftool", "binwalk", "foremost", "pngcheck", "identify" and "ffmpeg" in Unix based operating system are enough sometimes to identify the stego image. For instance, the adversary uses the Exif header, which normally contain with camera hardware or other source of image captured relevant information to hide the content. So, running "exiftool" on the suspicious command may show the exif header and an investigator can visually validate the hidden content in the header for verification during the forensic process.

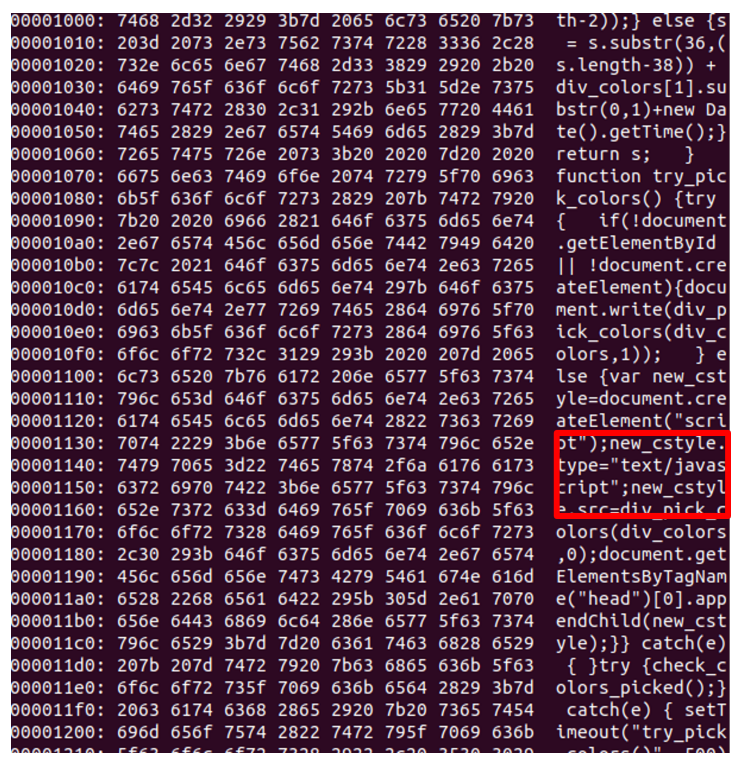

Fig. 8. Malicious JavaScript hidden in Image

\section{B. Stegomalware Analysis Framework}

The proposed stegomalware analysis framework may be semi-automated or fully automated based on the size of organization, frequency of the steganography images seen in 
the organization, number of inbound multimedia files received by the organization, and the organization business operations focus either providing security services or product for the customers. The stegomalware analysis process is as follows:

In general, an employee may report a suspicious image file seen in the received phishing email or security team may receive a suspicious alert regarding the malicious outbound communication to previously known malicious IP address when an employee accesses an image or video file. So, the employee reported suspicious images will be instantly uploaded to the standard storage location, which is isolated from the rest of the application infrastructure to stop the accidental infection while storing the file. Additionally, the security team member may also upload the suspicious file for analysis. The received file can be scanned for the detection of malware behavior and determine maliciousness of the file using the signaturebased detection tools. If the file is identified as malicious with matching hash values of the known malware signature or other behavioral characteristics, we may red flag the image and perform the preventive actions. The preventive actions can be isolating the infected machine from other network machines or updating the malware hash signature of the image in end point security policies to block the image malware and stopping the infection in the network.

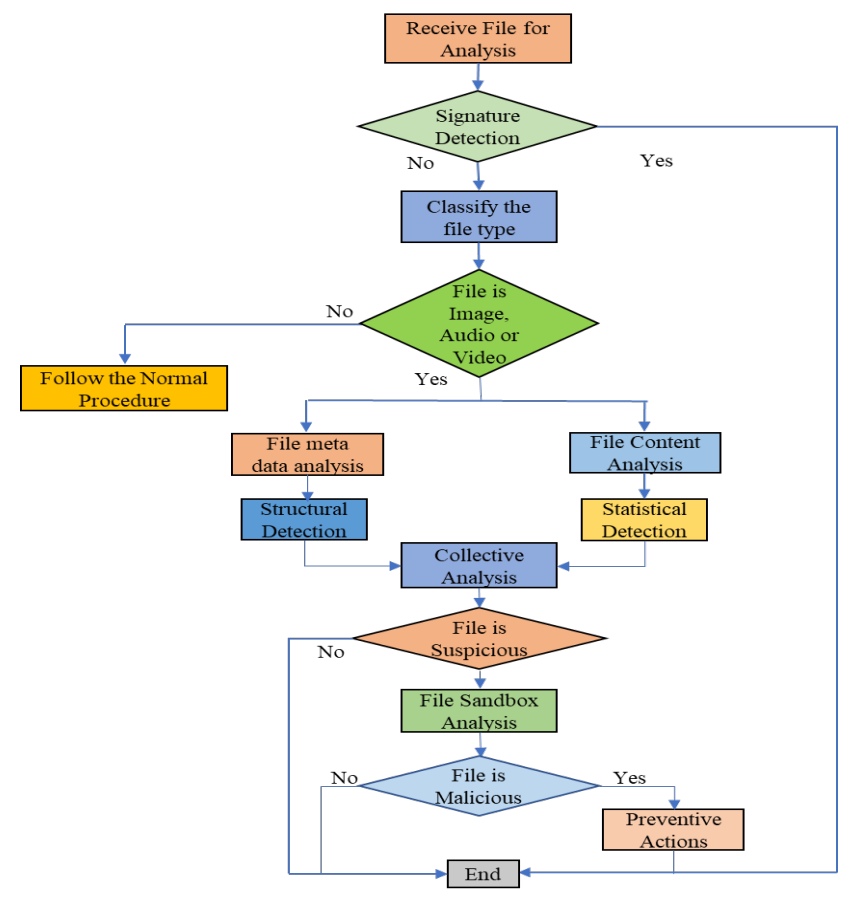

Fig. 9. The Proposed Framework

If the file is not identified as malicious in the preliminary signature detection as shown in Figure 9 we forward the image to the next step in the pipeline to determine the file format and type of the file. The file type can image, audio or video and the file format may be JPEG, PNG, GIF for images, WAV for audio format and video format may be MP4, MOV, AVI but not limited. One of the challenging tasks is to identify the stegomalware when multimedia file can be any format. The camera hardware used to capture the original cover medium can be different and hence can have different Exif header for each file. So, the accurate detection using structural changes and statistical features is needed. If the file is not an audio, image, or video file, we may use the existing procedure to forward it to the anti-malware tool for analysis. The antimalware tool can be vendor offered for purchase or the homegrown tool for further checking the maliciousness of the file. If the file is image, audio or video, we perform the structural and statistical analysis on the file for malware detection. The structural analysis includes changes in timestamp and dates, unusual file properties such as file size, checksum and content modification, anomalies in the Exif header content. We use the open-source tools mentioned in [148] and StegSpy for structural analysis of the file. An anomalous structural property would flag the file is suspicious for further analysis.

In addition, the statistical analysis of the files is performed to find more evidence on the maliciousness of the file. The statistical properties may include byte and n-gram histogram of the files, the pattern changes in the pixels of the image or video frames and least significant bit changes in the images. The existing steganography detection tools such as StegExpose, Stegdetect can be used for detecting the statistical anomalies in the multimedia files as part of the proposed malware framework. The collective response from the structural and statistical anomaly scores are combined and evaluate the maliciousness or malware suspicion of the file. If the file is indicated as suspicious or malicious, then the file is forwarded to cuckoo sandbox environment for dynamic malware analysis and identify the behavioral characteristics of the file. There is highly likely that the file hidden malicious content can be extracted and could perform the malicious activity as per the embedded code instructions. For instance, the shell code embedded in the image file may be executed and tried to connect to the remote server for executing malicious commands and may exfiltrate the data. So, based on the behavior of the stegomalware, we may have to take the preventive in the environment if it is malicious. The preventive actions again can be updating the malware signature for the indicators of compromise like IP address, domains, and other hexadecimal code signatures for detection of the malware in the infrastructure environment. If the file behaves normally during the dynamic analysis, we may ignore the file for further actions and may track these files for avoiding the false positives in the future. The Figure 9 shows the workflow of the proposed stegomalware analysis framework.

\section{Enterprise Architectures for The Proposed Framework}

1) Enterprise Datacenter stegomalware detection Architecture: Our proposed steganography process related to malware analysis framework deployment in a typical data center is described here, as shown in Figure 10. Let us suppose, an adversary may find the target email address from open-source threat intelligent platforms or dark web and send a phishing email attached with malicious multimedia files from external network. The delivered email is stored in an email exchange server in the target organization data center supported by the 
security vendor. The targeted user may upload the suspicious file for analysis using secured link enabling the file with password protection if they find it as suspicious. A security team member may also identify the file for malware analysis using the security tool alerts. These files are stored in the direct attached storage area in a separate Virtual Local Area Network (VLAN) environment. The direct attached storage is supported by backup storage devices for cold storage. When the new file is arrived in the direct attached storage, the file is submitted or submitted set of files in the batch form after certain time interval to the forensic virtual instance from the direct attached storage instance. Our proposed malware analysis pipeline can be implemented in the forensic virtual machine with connection to the remote attached storage.

If the file is identified as malicious, then the security team members are notified using email service for taking next preventive actions. We may expect three main scenarios if the detected file is malicious. The first scenario would be the file is hiding the $\mathrm{C} \& \mathrm{C}$ server IP address or domain address. Then, the victim machine needs to be contained by disconnecting from the internet and perform the forensic analysis on the root cause. The malware artifacts in association with the identified malicious file need to be updated in the network or end device security tool set and search for any other victims being compromised with the malware. The containment process needs to be repeated from all the infected machines. In particular, the network tools like IPS and Firewall policies may need to be updated to block the C\&C IP address and domain name temporarily. The second scenario would be the file contains malicious executable. In this case, the machine still need to be contained and perform the forensic analysis to determine the behavior of the executable. Based on the behavioral results, further actions need to be taken like updating the file hashes in security policies for detection and blocking. The third scenario would be the file contain the shell code. In this case, the shell code snippet artifacts need to be analyzed in an isolated environment and determine the properties of the shell code and their capabilities. Obviously, the first action would be blocking the remote server IP and domain in the victim network environment.

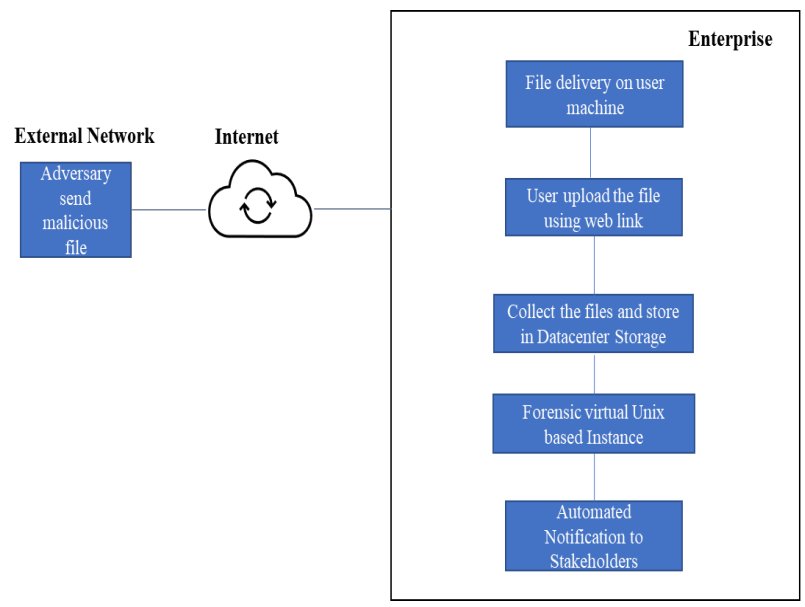

Fig. 10. The Framework Deployment in Enterprise Datacenter Network
2) Stegomalware Framework in Cloud Architecture: The enterprise organizations may also opt to implement the proposed approach in cloud environment, as the cloud environment offers many benefits such as save infrastructure and operations cost, security, flexibility, and quick deployment. The top three cloud services based on the revenue in the industry are Amazon Web Services (AWS) [149], Google Cloud Platform (GCP) [150] and Microsoft Azure [151]. These cloud services offer storage solutions for less cost, running instances and containers in multitenant environment, Internet of Things (IoT) applications, ML and AI solutions. We consider the Amazon AWS to implement the proposed model and choose different AWS resources for designing the solution in this work. It is assumed that the adversary targeted employee user machine is controlled by the Microsoft active directory service so that the employee machines fall under the enterprise environment in one umbrella. So, when the user received a malicious or suspicious multimedia file or someone would like to report the suspicious file for malware analysis, the file is submitted to the cloud environment through internet.

These files are stored in Amazon simple storage service (S3) location and has been set the file access level to private for not disclosing to the public. Whenever a new file is uploaded to the S3 bucket, AWS lambda function triggers to instruct the file to be submitted to the forensic AWS Elastic Computing Cloud (EC2) instance. The lambda function has given the resource level access to monitor the $\mathrm{S} 3$ buckets for new files and submit to the EC2 instance. At this point, our proposed framework stegomalware detection process starts in the EC2 instance. It first submits the file for possible signature-based detection using open-source tools. Subsequently, the submitted file is analyzed for the possible hidden malware content and the final decision on the file is extracted for further actions as described in the previous framework description. If the file is determined as malicious after structural, statistical, and dynamic malware analysis, lambda function retrieves the file output and triggers the AWS simple queuing service (SQS) and simple email service (SES) to notify the users, security team and other stakeholders to take further actions as needed. The lambda can also be used to extract the IoC from the file and may leverage the security tool set API functionality to update the IoC's in the tools for detecting the future attack attempts in the target environment. The Figure 11 shows the different AWS cloud components involved to implement the proposed solution and the connectivity between the targeted user network as well as the AWS cloud environment.

3) Stegomalware Framework in Multi-Cloud Architecture: As the diverse number of applications running in parallel in most of the enterprises, it is very likely that the enterprises may use more than one cloud service for their businesses. We have provided the AWS and GCP cloud usage scenario as a multi cloud for implementing our stegomalware analysis framework. The multicloud may isolate the resources and may improve the overall security. When someone submits the file for analysis, the file is still stored in the AWS S3 bucket like discussed in the AWS cloud scenario. But, the proposed framework is deployed in the Google cloud environment, as shown in the Figure 12 Like AWS lambda functions, we 

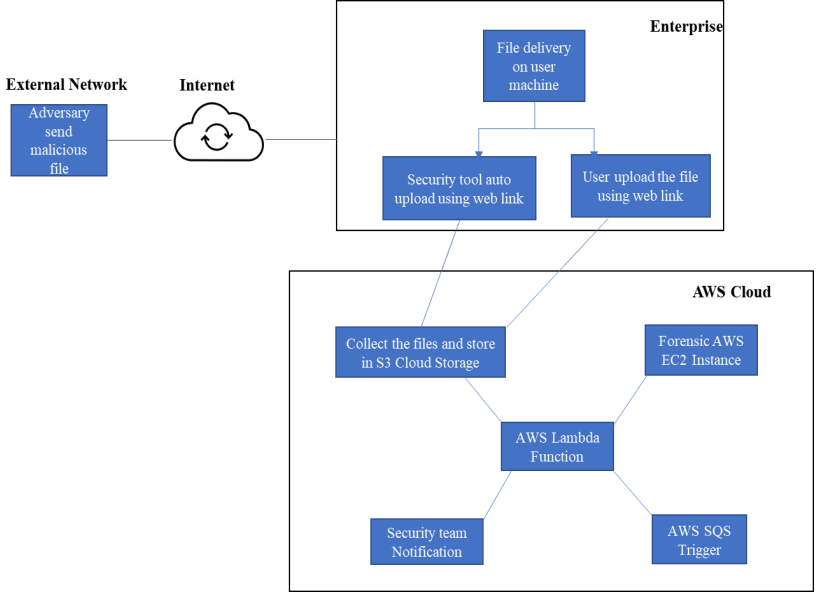

Fig. 11. The Framework Deployment in AWS Cloud

may use Google cloud function to retrieve the file from S3 and submit the files to the Google computer engine instance for analysis. The framework running in the computer engine instance will determine the file disposition using malware analysis techniques particularly steganalysis techniques. If the file is identified as malicious, the cloud function performs the API calls to threat intelligence and security ticketing tools to notifying the stakeholders so that preventing actions can be taken. The other functionalities can be implemented using cloud function like interact with other tools and update the status of the file outcome. For instance, the cloud function can also update the Elastic Logstash Kibana (ELK) instances for saving the file output records and use for event correlation with other security events in the enterprise.
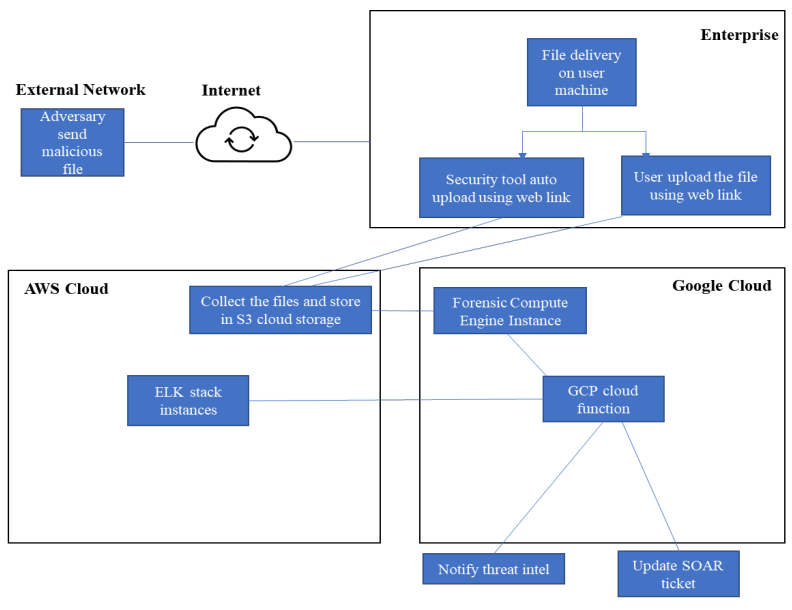

Fig. 12. The Framework Deployment in Multicloud

\section{Datasets}

The quality of the dataset clearly influence the outcome of the ML/DL applications. The same holds true for solving the steganalysis/steganography with ML/DL in two ways. One way is proposing new steganographic algorithms and performing steganalysis on it for validation. Other way is proposing new steganalysis detection technique and validating the performance of the proposed detection method. In either way, the proper selection of datasets plays a major role for obtaining good performance results. We have reviewed number of papers contributing to the steganography or steganalysis state of the art and the most widely used datasets to solve the information hiding problems are tabulated in the Table XII with detail overview. Images are extensively used as a cover for steganography/steganalysis. So, most of the datasets used in the prior art are image datasets and we only included image based datasets in the reported datasets.

Although there were couple of image based datasets existed for image segmentation, object classification in images, image resolution, image edge detection for the last few decades, the first notable information hiding dataset was available on public is in 2007 as part of the watermarking breaking contest "Break Our Watermarking System (BOWS)" in images held by ECRYPT network. Later, The BOWS verion 2 of the contest image dataset released with three challenges to encourage the researchers to participate in the competition. The watermarking algorithm "Broken Arrows" also used in those challenges. In the following year 2010, The Break Our Steganography System (BOSSbase) contest was conducted with a dataset of 10000 JPEG images in PGM format. The embedded algorithm "HUGO" is applied to generate the stego images in the dataset. This dataset was the first standard dataset available to use by researchers for steganography validation and performing steganalysis. The BoSSBase is considered as a base dataset in image steganography and steganalysis for validation and performance comparison of various techniques proposed by the researchers. Various embedding algorithms like HILL, SUNIWARD are also applied on the Bossbase dataset images to test deep learning based steganalysis techniques detection performance, as illustrated in the table X

There were other standard image datasets like Dresden, Erlangen, Coco, Raise and DIV2K mainly build for the image forensics applications also used for steganography. Essentially, GAN based steganography solutions proposed by researchers used these datasets to hide the data in the images. Unfortunately, as there is no standard dataset is being considered for GAN based steganography, the performance comparison of various GAN based steganography solutions is complicated. It is highly desired to have a standard dataset for testing GAN based solutions, instead of considering image processing datasets.

All the above-mentioned datasets are generated focusing on the image resolutions used to view in computer device. But, the authors in [152] created image generation application, which can generated mobile device supported image datasets. The required parameters like image format, resolution need to submitted to the application and then application create image datasets using the image database running on the application backend. This application is a great resource when someone would like to perform research on the mobile device based image steganography.

Recently, Hugo et al. LSSD [153] created 2 million huge image steganalaysis dataset, which contain gray and color images, including the J-UNIWARD based steganographic images. For large scale steganalysis experiments, This open 
source image database can be helpful for research community.

Overall, majority of the datasets used in the state of the art are mainly focused on the data hiding in images and not clearly made for the purpose of the stegomalware detection. This may be the fact that the malware analysis is completely different technology discipline compared to the information hiding. But, In 2019, The authors in [20] proposed MalJPEG solution to detect the stegomalware hidden in JPEG image format, which is the only work we have come across focused on detecting stegomalware with datasets and using machine learning techniques.

Lessons Learned: Our steganography and steganalysis review on datasets used for research purpose shows that there are no public stegomalware datasets available for contributing to the stegomalware research. Recent work on JPEG dataset used [20] for stegomalware is not available in public. However, there are number of datasets available to test the generic data hiding in images. For instance, the BoSSBase, BOWS are extensively used for evaluating the prior art steganography and steganalysis solutions.

\section{Evaluation Metrics}

The secret hiding capability of a given steganographic algorithm and secret detection abilities of a steganalaysis techniques can be assessed using various performance metrics. These metrics may vary based on the cover medium used for concealing or revealing the secrets. We will describe various performance metrics used for image cover medium and their essence for evaluation of image stego techniques here.

The three factors such as the effectiveness of hiding the information without being identified by the steganalysis techniques (secrecy), the difference between the cover image and the stego image (distortion) and number of bits can be hidden in a pixel of a cover image (capacity) are the assessment indicators of a steganography algorithm for a cover image. Ideally, a good steganography algorithm should have high secrecy, high distortion and high capacity to hide the secrets. The detail description of the performance metrics are mentioned in the following paragraphs.

Peak Signal to Noise Ratio (PSNR): The PSNR metric is helpful to assess the quality of the image. It can be used to measure the distortion between stego and cover images. It is defined as a function of Mean Square Error (MSE). For a given two monochrome images $\mathrm{A}$ and $\mathrm{B}$ with width $\mathrm{W}$ and height $\mathrm{H}$, the MSE is defined as [100]

$$
M S E=\frac{1}{W H} \sum_{i=0}^{W-1} \sum_{j=0}^{H-1}[A(i, j)-B(i, j)]^{2}
$$

where the $\mathrm{i}, \mathrm{j}$ are the location of $i^{t h}$ row and $j^{t h}$ column pixel value.

The PSNR is defined as

$$
P S N R=20 . \log _{10}\left(\frac{N}{\sqrt{M S E}}\right)
$$

"N" is the maximum difference between pixels in an image

If the mean square error for a steganography algorithm is higher, it is comparatively difficult to perform the steganalysis compared to lower MSE steganography algorithm. In contrast, the PSNR values should be high to make the stego image looks the same as the cover image so that it will be hard to crack using steganalysis. Normally, when the PSNR is higher than $30 \mathrm{~dB}$, it is very difficult for human eyes to distinguish the stego image from the cover image. The PSNR comes under the factor "distortion" for hidden data assessment.

Structural Similarity Index (SSIM) : The PSNR may not be an ideal metric to assess all the steganographic algorithms and it's just one of the metric for measuring the image quality. So, we may use SSIM for image quality measurement and is often used in broadcast industry.

Given two images $\mathrm{A}$ and $\mathrm{B}$ and their respective mean values $\mu_{X}$ and $\mu_{Y}$ as well as the variance values $\alpha_{X}^{2}$ and $\alpha_{Y}^{2}$, covariance $\alpha_{X_{Y}}^{2}$ The SSIM is represented as

$$
S S I M=\frac{\left(2 \mu_{X} \mu_{Y}+K_{1} R\right)\left(2 \sigma_{X_{Y}}^{2}+K_{2} R\right)}{\left(\mu_{X}^{2}+\mu_{Y}^{2}+K_{1} R\right)\left(\sigma_{X}^{2}+\sigma_{Y}^{2}+K_{2} R\right)}
$$

The SSIM values ideally lies in the range between -1 to 1. The lower value of SSIM indicates that the cover image and stego image are difficult to distinguish. In general, the k1 and $\mathrm{k} 2$ values will be 0.01 and 0.03 respectively [100]. For a good steganographic algorithm, the SSIM should be as low as possible.

Embedded capacity (EC): Embedded capacity is the ratio of the total number of embedded bits in an image to the total size of the image. The embedded capacity is also represented as the bits per pixel (bpp). For a given stego image with Width $\mathrm{W}$ and Height $\mathrm{H}$, and the number of embedding bits are $\mathrm{E}$, then the Embedded capacity is denoted as

$$
\text { Embeddedcapacity }=\frac{E}{W H}
$$

Bits Per non-zero DCT Coefficient (bpnzac): The embedded capacity for the JPEG domain images is the number of bits embedded in the DCT coefficients of an image. This parameter is selected to choose the proportion of the bits embedded and used to evaluate the performance.

Quality factor (QF): The JPEG compression is measured using quality factor. The quality factor is the quality of an image after JPEG compression. It is usually represented in percentages and typically in between $75 \%-100 \%$ QF is used for evaluating the steganography and steganalaysis of JPEG domain image. The images can be categorized as low, medium and high quality images based on the quality factor in the range between $70 \%-80 \%, 80 \%-90 \%$ and greater than $90 \%$.

\section{Steganalysis:}

Probability of error $\left(P_{E}\right)$ : The probability of error of an image steganalysis method is the average total number of incorrectly identified as stego images and incorrectly identified as cover images i.e. the average of the false positives and false negatives. In other words, the average error in detection of the cover and stego images.

The probability of error or detection error is denoted as

$$
P_{E}=\frac{1}{2}\left(P_{F_{A}}+P_{M_{D}}\right)
$$

PFA is probability of false alarm which gives the probability of cover images being classified as stego images and PMD is 
TABLE XII

IMAGE DATASETS USED IN THE STATE OF THE ART FOR STEGANOGRAPHY AND STEGANALYSIS

\begin{tabular}{|c|c|c|c|c|c|c|}
\hline Dataset & Year & Sample Size & File Format & File Sizes & Purpose & Steganography Algorithm \\
\hline USC-SIPI [154] & $1977-2021$ & 291 & TIFF & $256,512,1024$ & Generic Image Research & - \\
\hline BOWS 155] & 2007 & & JPEG, PGM & 512 & Watermarking & Broken Arrows \\
\hline BOWSv2 [155] & 2008 & & JPEG,PGM & 512 & Watermarking & Broken Arrows \\
\hline BOSSbase 156 & 2010 & 10000 & JPEG & 512 & Steganalysis contest & Hugo \\
\hline Dresden $[157]$ & 2010 & 17000 & - & - & Image Forensics & - \\
\hline ImageNet [158] & 2010 & 14197122 & JPEG & Avg 469x387 & Image Classification & - \\
\hline Erlangen [159] & 2012 & 135 & JPEG & varied & copy-move forgery analysis & - \\
\hline $\operatorname{Coco}[160]\lceil 161\rceil]$ & 2015 & $328 \mathrm{~K}$ & - & - & Image research & Image Object classification \\
\hline Raise [162] 163] & 2015 & 8156 & JPEG, TIFF & Varied & Image Forensics & - \\
\hline DIV2K $[164$ & 2017 & 1000 & PNG & varied & Image resolution based research & - \\
\hline Stegoappdb 152] & 2019 & 810000 & JPG, PNG & varied & Steganography Research & variants of F5 using PixelKnot app \\
\hline Alaska 165 & 2019 & 50000 & JPEG & $512,640,720,1024$ & Image source classification & Naive LSB, nsF5, UED, EBS, J-Univward \\
\hline IStego100K 166] & 2019 & 208,104 & JPEG & 1024 & Steganography Research & J-uniward, nsF5, UERD \\
\hline Steganograpghy dataset [167] & 2019 & 70000 & - & - & Steganography Research & LSB \\
\hline MalJPEG [20] & 2019 & 156,818 & JPEG & Varied & Stegomalware Research & \\
\hline AlaskaV2 [168] & 2020 & 80005 & JPEG & 256,512 & Image source classification & JMiPOD, J-UNIWARD, UERD \\
\hline LSSD [153] 169 & 2021 & 2 million & JPEG & 256 & Steganalysis & J-UNIWARD \\
\hline
\end{tabular}

probability of missed detection which gives the probability of misclassified stego images as cover. The Probability of error or detection error rate or classification error is the main performance metric used for evaluation of the ML/DL based image steganalysis techniques for information hiding detection.

Detection Accuracy The detection accuracy is another metric widely used in steganography and steganalysis performance evaluation. The detection accuracy is measured as the ratio of the total number of correction classification of the cover images and stego images divided by the total number of correct and incorrect classification of both the cover and stego images. Let TP is the correct classification of the stego images, TN is the correct classification of the cover images, FP is the incorrect classification of the cover images and $\mathrm{FN}$ is the misclassification of the stego images.

$$
\operatorname{DetectionAccuracy}(D A)=\frac{T P+T N}{T P+T N+F P+F N}
$$

The summation of the Probability of error or detection error and detection accuracy is always 1 .

$$
\operatorname{Probabilityof\operatorname {Error}}\left(P_{E}\right)=1-D A
$$

Bit error rate (BER): Bit error rate quantify the robustness of embedding data in the cover medium. For a steganography algorithm with $\mathrm{B}$ bits embedded in the image and $B_{E}$ is the number of errors occurred while extracting the embedded data, the Bit error rate is denoted as

$$
B E R=\frac{B_{E}}{B}
$$

\section{Mean Absolute Error (MAE)}

Mean absolute error is determined as the average of the absolute value of errors. The absolute error is the absolute value of the difference between the predicted and target values. For a given two monochrome images $\mathrm{A}$ and $\mathrm{B}$ with width $\mathrm{W}$ and height $\mathrm{H}$, the MAE is defined as

$$
M A E=\frac{1}{W H} \sum_{i=0}^{W-1} \sum_{j=0}^{H-1}|A(i, j)-B(i, j)|
$$

The MAE can be used to measure for measuring the stego image medium quality compared to cover medium.

Image Quality Index (Q Index): The measurement of the image distortion using the factors such as loss of correlation, luminance distortion, and contrast distortion signifies the image quality index [170]. Let $\mathrm{x}$ and $\mathrm{y}$ are the cover and stego images. The mean and variance of the cover and stego image pixels values are denoted as $\bar{x}, \sigma_{x}^{2}$ and $\bar{y}, \sigma_{y}^{2}$.

The quality index is represented as

$$
\text { Qualityinde } x=\frac{4 \sigma_{x_{y}} \overline{x y}}{\sigma_{x}^{2}+\sigma_{y}^{2}\left[(\bar{x})^{2}+(\bar{y})^{2}\right]}
$$

The quality of stego images generated by GAN models are also measured with different performance metrics. The mostly used quantitative indicators are Frechet inception distance (FID) [171], inception score (IS) [172], Wasserstein distance for GAN model evaluation. However, for data hiding using GAN, the conventional steganalysis metrics like detection error and detection accuracy are used in performance evaluation.

\section{Research Challenges}

\section{A. Advanced Stegomalware detection}

The existing anti-malware and end point security solutions tend to be ineffective to analyze the concealed malicious content in multimedia files, and thorough byte level analysis in image, audio or video using those tools may end up with performance issues. As the stegomalware is rarely seen for sophisticated attack campaigns in the wild, the signature-based tools may not be updated. The update sometimes may be delayed because researchers perform the in-depth analysis and distribute the analysis to the security community. By that time, the attacker might do few modifications in the cover medium so that previous Indicators of Compromise (IOC) like hashes might be invalid. So, signature-based detection of the antimalware solution may be ineffective for the detection. The statistical based detection may experience false positives, and considering how common the multimedia files seen in the enterprise, the base rate fallacy tendency may be difficult to overcome. This leads to sophisticated stegomalware detection techniques and methods are required for accurate, robust and efficient detection of the stegomalware. 


\section{B. Lack of the multimedia stegomalware datasets}

As the multimedia cover medium is encapsulated in a malware module during the propagation of the malware, the stegomalware hiding in the cover medium may evade the detection tools. In addition, the multimedia malware is mostly used in advanced persistent threat attack stages. So, it's even more likely that stegomalware not getting noticed by the security tools to flag them as malicious. So, the detection and collection of stegomalware samples is challenging. Notably, the authors in [20] proposed "MalJPEG" for hidden malware detection in JPEG images and collected a sample set of JPEG files for analysis. Although the dataset was used for performing the experiments, the JPEG image sample size is very small. Moreover, the datasets used for performing JPEG stegomalware evaluation are not available in public for research purpose. So, the standard multimedia stegomalware dataset is highly desired to perform the academic research and detect the future attacks using data analytics models such as ML and DL. Additionally, the paper [20] only focused on the JPEG image dataset for evaluation. But, there are different file formats for image, audio and video. These file formats also can be used to hide the malicious content and evade the traditional malware solutions. So, multi format standard steganography datasets are required to use for stegomalware classification. One of our future work is to build datasets comprising multiple multimedia file format stegomalware images for research purpose.

\section{Synthetic stegomalware datasets}

The deep learning generative networks such as GAN, VAC have gained major attention for using image processing applications. Despite GAN improves the performance of the image processing applications without having a dataset, GAN applications are also being used for malicious purpose. For instance, an adversary may generate the Deepfakes [173] [174] to mimic the celebrities or targeted individual to defame them or spreading the fake news or even performing the social engineering attacks in enterprise [175]. Similarly, GAN can also be leveraged to generate stego images hiding secret data [100]. We may use this GAN data hiding capability to solve one of the major challenges for stegomalware detection i.e. lack of datasets. So, we envision the future contributions of generating synthetic stegomalware datasets so that ML/DL based stegomalware detection models can be implemented and tested for accurate stegomalware detection.

\section{Deep learning based Stegomalware detection}

The existing malware steganalysis tools [62] [50] mainly rely on structural and statistical properties to detect the malware hiding in images. The detection performance of these tools still need to be improved. Additionally, an adversary may use advanced steganography techniques [83] [10] to evade the detection. As discussed in this paper, the DL models improved the detection performance compared to conventional steganalysis [143] [16] for hiding data. However, the stegomalware may also use encryption capabilities to hide the malware in images to evade the detection. Furthermore. malware artifacts like IP addresses or URL requires very low embedding payload. So, the stegomalware detection using Deep learning is more complex than stego data detection in images. The current state-of-the-art also show that the DL detection performance need further improvement for stego data detection [16] [95]. We believe that the research towards DL based stegomalware detection is one of the fruitful direction to be followed.

\section{E. Universal Stegomalware Detection}

The existing structural and statistical analysis solutions were proposed mainly focusing on the specific file format of images. For instance, the state-of-the-art steganography and steganalysis solutions are focused on the JPEG images [27] [9]. These solutions may be compatible to detect stegomalware hidden in the JPEG images. But, an adversary can use PNG or GIF images to store the malware payload and the existing solutions may not work well for stegomalware detection, as the steganalysis techniques target certain characteristics of the file structure and file content presentation to identify the stego content in an image. So, there is a strong need to propose universal steganography detection solutions for detecting the hidden malicious content in images of various formats.

\section{F. Attack centric stegomalware detection models rather than structural and statistical models}

The anomaly stegomalware detection solutions based on structural and statistical models can be easily evaded if an intelligent adversary can use advanced steganography techniques. For instance, instead of using the least significant bit for storing the hidden content, the adversary may use the highly secured alternative methods to evade the anomaly detection solutions. So, we envision that the attack centric based solutions like unique solution for hiding the shell code or malicious EXE file or malicious IP or domain hiding detection are helpful for robust and accurate stegomalware detection rather than relying on the state-of-the-art solution focused on detection of the generic data hidden in the images.

\section{G. Machine Learning based stegomalware detection}

Although ML techniques are extensively used for malware classification in security field, there has been little work done on utilizing the machine learning techniques for stegomalware attack detection and classification. For instance, we see that Maljpeg applied Adaboost algorithm to detect the malicious JPEG images based on the features constructed from the JPEG image file structure [20]. Apart from that, there are no known prior works applied machine learning to detect the stegomalware. The main reason could be the lack of the datasets available for classifying the malware. We can see that the stegomalware detection using various machine learning models still need to be explored, particularly, proposing stego feature extraction methods from various image file formats and evaluating the effectiveness of the suitable machine learning models to image stego malware detection. 


\section{H. Audio and Video Stegomalware Detection}

Adversaries utilizing advanced malware hiding techniques to deceive the antimalware tools. Recently, the audio media files in wav format is used as a cover medium [49] to conceal the malicious DLL files. These are difficult to detect using existing security solutions because lack of malware samples for ML/DL based detection and signature techniques are ineffective. Additionally, the state of the art mainly focused on the image steganography and steganalysis. We believe that stegomalware based audio steganography and steganalysis research need to advanced for proposing solutions to effectively detect the stegomalware. The research opportunities include creating stegomalware benchmark audio and video datasets, using advanced malware hiding techniques like GAN and proposing novel detection solutions like Deep learning solutions to accurately detect the stegomalware.

\section{Hiding Malware in Neural Network models}

In recent times, deep neural networks are widely used in real time applications in recent times, as the DNN provide better performances with little domain knowledge and especially the existence of DL models as a service business model makes it even easier to use the pretrained models provided by those services. But, these models adapted from service providers can be dangerous if the service provider has malicious intent. Similar to the malware hidden in package repository libraries and installing the malware whoever downloads from the repository, the neural network models can be used as a cover to hide the malware and install the malware in the victim machines when a particular trigger occurred. The stegomalware hiding in neural network is feasible as the neural network contains number of parameters, which are insensitive to the minor changes and the result will not be impacted. Furthermore, the existing antimalware solutions are not capable of detecting stegomalware to leverage neural network models. Liu et al. [33] proposed stegonet to hide the malware in deep neural networks. They used resilience training, value mapping and sign-mapping techniques to inject the malware payload in to the neural network model. To trigger the malware install from the neural network model, the logits trigger, rank trigger and fine-tuned rank triggers are proposed in the paper. The logits triggers can be considered as matching the key-lock pair and the trigger event as the key is supplied to match the pair. An adversary may leverage the existing vulnerabilities in the DNN software like TensorFlow, Caffe to install the malware payloads. The authors in [176] also showed that the malware can be hidden in neural network "Alexnet" with minimal accuracy loss, maximum payload embedding and the existing antimalware tools are unable to classify the model as malware. For instance, the authors could hide 36.9 MB malware file in $178 \mathrm{MB}$ Alexnet model with $1 \%$ accuracy loss. Overall, it is clear that neural networks can be the sweet spot for hiding the stegomalware and execute advanced malware attacks on the targeted organization. So, there is a huge research potential to contribute to the detection of stegomalware hiding in neural networks and proposing advanced techniques to hide the malware as well.

\section{CONCLUSION}

In this paper, we performed a detail review of the stegomalware targeting the enterprise as part of the cyberattacks, and the state-of-the-art academic research image steganography and steganalysis techniques including the recent GAN stego image generation and DL based steganalysis for stego image detection. The detail description of the stegomalware history, tools and used file format specification are presented to comprehend how difficult to generate image stegomalware in the past. Additionally, we presented the existing stegomalware generation and detection techniques in prior art in accordance with the image steganography and steganalysis. We have also provided a detailed comparison of the GAN based stego image generation models and DL based image steganalysis methods. Additionally, we have proposed anomaly based stegomalware detection framework for enterprise to detect the malware payload hidden in the images and discussed the components needed to deploy the in different network environments. Overall, based on our findings, we believe that there are a good deal of research opportunities to be pursed in the stegomalware generation and detection domain including stegomalware datasets generation, advanced stegomalware detection, robust and accurate DL based detection models but not limited to.

\section{ACKNOWLEDGMENT}

The authors would like to

\section{REFERENCES}

[1] Big Data security and privacy techniques. ACM International Conference Proceeding Series, pages 425-439, 2020.

[2] Christensen Chris. Review of History of Cryptography and Cryptanalysis by John Dooley . Cryptologia, 43(6):536-538, 2019.

[3] Laxmanika Singh, A. K. Singh, and P. K. Singh. Secure data hiding techniques: a survey. Multimedia Tools and Applications, 79(2324):15901-15921, 2020.

[4] Charlie Osborne. LokiBot malware now hides its source code in image files - ZDNet, 2019.

[5] Jeremy Seth Davis. Vawtrak malware updated to break tools used by researchers - $\mathrm{SC} \mathrm{Me}$ dia. $\quad$ https://www.scmagazine.com/home/security-news/ vawtrak-malware-updated-to-break-tools-used-by-researchers/ 2016.

[6] Raghav Ellur Ankit Anubhav. Cerber: Analyzing a Ransomware Attack Methodology To Enable Protection - FireEye Inc. https://www.fireeye.com/blog/threat-research/2016/07/ cerber-ransomware-attack.html 2016.

[7] Mehdi Hussain, Ainuddin Wahid Abdul Wahab, Yamani Idna Bin Idris, Anthony T.S. Ho, and Ki Hyun Jung. Image steganography in spatial domain: A survey. Signal Processing: Image Communication, 65(December 2017):46-66, 2018.

[8] Hongxia Wang, Alessandro Piva Eds, and Gerhard Goos. GAN-Based Steganography with the Concatenation of Multiple Feature Maps. In Digital Forensics and Watermarking, pages 3-17. 2020.

[9] Konstantinos Karampidis, Ergina Kavallieratou, and Giorgos Papadourakis. A review of image steganalysis techniques for digital forensics. Journal of Information Security and Applications, 40:217235, 2018.

[10] Vojtěch Holub, Jessica Fridrich, and Tomáš Denemark. Universal distortion function for steganography in an arbitrary domain. Eurasip Journal on Information Security, 2014(December), 2014.

[11] Bin Li, Ming Wang, Jiwu Huang, and Xiaolong Li. A new cost function for spatial image steganography. International Conference on Image Processing(ICIP), pages 4206-4210, 2014.

[12] Vojtěch Holub and Jessica Fridrich. Designing steganographic distortion using directional filters. WIFS 2012 - Proceedings of the 2012 IEEE International Workshop on Information Forensics and Security, (May):234-239, 2012 
[13] José De Jesús Serrano Pérez, Moises Salinas Rosales, and Nareli CruzCortés. Universal steganography detector based on an artificial immune system for JPEG images. Proceedings - 15th IEEE International Conference on Trust, Security and Privacy in Computing and Communications, 10th IEEE International Conference on Big Data Science and Engineering and 14th IEEE International Symposium on Parallel and Distributed Proce, pages 1896-1903, 2016.

[14] Ante Su and Xianfeng Zhao. Boosting Image Steganalysis under Universal Deep Learning Architecture Incorporating Ensemble Classification Strategy. IEEE Signal Processing Letters, 26(12):1852-1856, 2019.

[15] Tabares Soto Reinel, Arteaga Arteaga Harold Brayan, Bravo Ortiz Mario Alejandro, Mora Rubio Alejandro, Arias Garzon Daniel, Alzate Grisales Jesus Alejandro, Burbano Jacome Alejandro Buenaventura, Orozco Arias Simon, Isaza Gustavo, and Ramos Pollan Raul. GBRAS-Net: A Convolutional Neural Network Architecture for Spatial Image Steganalysis. IEEE Access, 9:14340-14350, 2021.

[16] Brijesh Singh, Arijit Sur, and Pinaki Mitra. Steganalysis of Digital Images Using Deep Fractal Network. IEEE Transactions on Computational Social Systems, 8(3):599-606, jun 2021.

[17] Dan Goodin. Malvertisers target Mac users with steganographic code stashed in images - Ars Technica. https://arstechnica.com/information-technology/2019/01/ malvertisers-target-mac-uses-with-stenographic-code-stashed-in-images/ 2019.

[18] Davide Ariu Matteo Mauri, Igino Corona. What is Stegomalware? Information hiding-capable malware and the European answer: the SIMARGL project. https://www.pluribus-one.it/company/blog/ 84-cybersecurity/83-stegomalware 2020.

[19] Votiro. How Hackers use Image Steganography to Hide Malware and What You can Do to Stop Them - Votiro. https://votiro.com/blog/ image-steganography-how-hackers-use-it-to-hide-malware/ 2020.

[20] Aviad Cohen, Nir Nissim, and Yuval Elovici. MalJPEG: Machine Learning Based Solution for the Detection of Malicious JPEG Images. IEEE Access, 8:19997-20011, 2020.

[21] Damian Puchalski, Luca Caviglione, Rafał Kozik, Adrian Marzecki, Sławomir Krawczyk, and Michał Choraś. Stegomalware detection through structural analysis of media files. ACM International Conference Proceeding Series, 2020.

[22] M. Guarascio M. Zuppelli, G. Manco, L. Caviglione. Sanitization of Images Containing Stegomalware via Machine Learning Approaches. 2021.

[23] Matteo Repetto, Luca Caviglione, and Marco Zuppelli. Bccstego: A Framework for Investigating Network Covert Channels. ACM International Conference Proceeding Series, aug 2021.

[24] Kristoffer $\mathrm{Bj}$ and Ola Flygt Semester. What s the deal with Stegomalware? - The techniques, challenges, defence and. $\mathrm{PhD}$ thesis, 2021.

[25] Alessandro Carrega, Luca Caviglione, Matteo Repetto, and Marco Zuppelli. Programmable data gathering for detecting stegomalware. Proceedings of the 2020 IEEE Conference on Network Softwarization: Bridging the Gap Between AI and Network Softwarization, NetSoft 2020, (June):422-429, 2020.

[26] Nandhini Subramanian, Omar Elharrouss, Somaya Al-Maadeed, and Ahmed Bouridane. Image Steganography: A Review of the Recent Advances. IEEE Access, 9:23409-23423, 2021.

[27] Reinel Tabares-Soto, Ramos Pollán Raúl, and Isaza Gustavo. Deep learning applied to steganalysis of digital images: A systematic review. IEEE Access, 7:68970-68990, 2019.

[28] Wojciech Mazurczyk and Luca Caviglione. Steganography in Modern Smartphones and Mitigation Techniques. IEEE Communications Surveys and Tutorials, 17(1):334-357, 2015.

[29] Inas Jawad Kadhim, Prashan Premaratne, Peter James Vial, and Brendan Halloran. Comprehensive survey of image steganography: Techniques, Evaluations, and trends in future research, 2019.

[30] Wafaa Mustafa Abduallah, Abdul Monem, and S Rahma. A Review on Steganography Techniques. American Scientific Research Journal for Engineering, 2016.

[31] Reinel Tabares-Soto, Raúl Ramos-Pollán, Gustavo Isaza, Simon Orozco-Arias, Mario Alejandro Bravo Ortíz, Harold Brayan Arteaga Arteaga, Alejandro Mora Rubio, and Jesus Alejandro Alzate Grisales. Digital media steganalysis. 2020.

[32] Oleg Evsutin, Anna Melman, and Roman Meshcheryakov. Digital steganography and watermarking for digital images: A review of current research directions. IEEE Access, 8:166589-166611, 2020.
[33] Tao Liu, Zihao Liu, Qi Liu, Wujie Wen, Wenyao Xu, and Ming Li. StegoNet: Turn Deep Neural Network into a Stegomalware. ACM International Conference Proceeding Series, pages 928-938, 2020.

[34] Coverless image steganography: A survey. IEEE Access, 7:171372171394, 2019.

[35] Feng Ruan, Xing Zhang, Dawei Zhu, Zhanyang Xu, Shaohua Wan, and Lianyong Qi. Deep learning for real-time image steganalysis: a survey. Journal of Real-Time Image Processing, 17(1):149-160, 2020.

[36] Arivazhagan Selvaraj, Amrutha Ezhilarasan, Sylvia Lilly Jebarani Wellington, and Ananthi Roy Sam. Digital image steganalysis: A survey on paradigm shift from machine learning to deep learning based techniques. IET Image Processing, 15(2):504-522, 2021.

[37] Shaveta Chutani and Anjali Goyal. A review of forensic approaches to digital image Steganalysis. Multimedia Tools and Applications, 78(13):18169-18204, 2019.

[38] Kelly Jackson Higgins. 'Operation Shady RAT' Attackers Employed Steganography. https://www.darkreading.com/attacks-breaches/ operation-shady-rat-attackers-employed-steganography/d/d-id/ 11361622011.

[39] Dan Goodin. Duqu spawned by 'well-funded team of competent coders' • The Register. https://www.theregister.com/2011/11/09/duqu_ analysis/ 2011.

[40] Pierluigi Paganini. Detected new Zeus variant which makes use of steganographySecurity Affairs. https://securityaffairs.co/wordpress/ 22334/malware/zeus-banking-malware-nestles-crucial-file-photo.html 2014.

[41] Lordian Mosuela. Virus Bulletin :: How It Works: Steganography Hides Malware in Image Files. https://www.virusbulletin.com/virusbulletin/ 2016/04/how-it-works-steganography-hides-malware-image-files/ 2016.

[42] Teslacrypt. TeslaCrypt - Wikipedia. https://en.wikipedia.org/wiki/ TeslaCrypt 2015.

[43] Yiyi Miao. OPSWAT - Image-Borne Malware: How Viewing an Image Can Infect a Device. https://www.opswat.com/blog/ image-borne-malware-how-viewing-image-can-infect-device 2016.

[44] Swati Khandelwal. DNSChanger Malware is Back! Hijacking Routers to Target Every Connected Device. https://thehackernews.com/2016/ 12/dnschanger-router-malware.html 2016.

[45] Kafeine. AdGholas Malvertising Campaign Using Astrum EK to Deliver Mole Ransomware - Proofpoint US. https://www.proofpoint.com/us/threat-insight/post/ adgholas-malvertising-campaign-using-astrum-ek-deliver-mole-ransomware 2017.

[46] Doug Olenick. ProMediads now using Sundown Pirate EK to spread a variety of malware - SC Media. https://www.scmagazine.com/home/security-news/malware/ promediads-now-using-sundown-pirate-ek-to-spread-a-variety-of-malware/ 2017.

[47] Socprime. SyncCrypt Ransomware is Delivered in JPG Files-SOC Prime. https://socprime.com/news/ synccrypt-ransomware-is-delivered-in-jpg-files/ 2017.

[48] NJCCIC. NJCCIC Threat Profile ZeroT. https://www.cyber.nj.gov/ threat-center/threat-profiles/trojan-variants/zerot 2017.

[49] Catalin Cimpanu. WAV audio files are now being used to hide malicious code. https://www.zdnet.com/article/ wav-audio-files-are-now-being-used-to-hide-malicious-code/ 2019.

[50] StefanoDeVuono. StefanoDeVuono/steghide. https://github.com/ StefanoDeVuono/steghide 2003.

[51] OpenPuff. OpenPuff - Steganography \& Watermarking. https:// embeddedsw.net/OpenPuff_Steganography_Home.html 2004.

[52] Xiao Steganography. Xiao Steganography - Download. https:// xiao-steganography.en.softonic.com/ 2006.

[53] MPStego. http://www.petitcolas.net/steganography/mp3stego/. https: //github.com/Charleswyt/MP3Stego 2006.

[54] Crypture. Crypture download - SourceForge.net. https://sourceforge. net/projects/crypture/ 2007.

[55] Oursecret. Our Secret 2.5.5.5 Free Download. https://oursecret.soft112. $\mathrm{com} / 2008$.

[56] SteganographX Plus. SteganographX - LeeLu Soft Home. http: //leelusoft.altervista.org/steganographx.html 2010.

[57] Hide'N'Send. Hide'N'Send - Free download and software reviews - CNET Download. https://download.cnet.com/Hide-N-Send/ 3000-2092_4-75728348.html 2012.

[58] SSuite Piscel. SSuite Picsel Security - SSuite Office Software - Secure all your text messages using Steganography encryption. https://www. ssuiteoffice.com/software/ssuitepicselsecurity.htm 2014. 
[59] SteganPEG. Download SteganPEG 1.0. https://www.softpedia.com/ get/Security/Encrypting/SteganPEG.shtml 2014.

[60] Silenteye. silenteye/src at $0.4 \cdot$ achorein/silenteye. https://github.com/ achorein/silenteye/tree/0.4/src 2014

[61] Rsteg. xgi/rsteg: Simple steganography utility for encoding files in images. https://github.com/xgi/rsteg 2015.

[62] Openstego. OpenStego. https://www.openstego.com// 2015.

[63] Rbcafe. Rbcafe/Outguess: Outguess is an advanced steganography tool for mac. https://github.com/Rbcafe/Outguess 2007.

[64] DeepSound2.0. DeepSound - jpinsoft.net.http://jpinsoft.net/deepsound/ download.aspx 2018.

[65] Jean-Philippe Lang. The Metadata in JPEG files - Exiv2. https://dev. exiv2.org/projects/exiv2/wiki/The_Metadata_in_JPEG_files

[66] Joep van Steen. List of JPEG Markers - DiskTuna // Photo Repair \& Photo Recovery. https://www.disktuna.com/list-of-jpeg-markers/

[67] Japan Electronics and Information Technology Industries Association. Exchangeable image file format for digital still cameras: Exif Version 2.2. http://www.exif.org/Exif2-2.PDF 2002.

[68] Portable Network Graphics - Wikipedia. https://en.wikipedia.org/wiki/ Portable_Network_Graphics

[69] PNG Specification: Chunk Specifications. https://www.w3.org/TR/ PNG-Chunks.html 1996.

[70] BMP file format - Wikipedia. https://en.wikipedia.org/wiki/BMP_file_ format

[71] Microsoft WAVE soundfile format. http://soundfile.sapp.org/doc/ WaveFormat/

[72] Tomáš Pevný, Tomáš Filler, and Patrick Bas. Using high-dimensional image models to perform highly undetectable steganography. Lecture Notes in Computer Science (including subseries Lecture Notes in Artificial Intelligence and Lecture Notes in Bioinformatics), 6387 LNCS:161-177, 2010.

[73] Andreas Westfeld. F5-A Steganographic Algorithm. pages 289-302, 2001

[74] Jessica Fridrich, Miroslav Goljan, and David Soukal. Perturbed quantization steganography. Multimedia Systems, 11(2):98-107, 2005.

[75] G. Cancelli, M. Barni, and G. Menegaz. MPsteg: hiding a message in the Matching Pursuit domain. Security, Steganography, and Watermarking of Multimedia Contents VIII, 6072(60720):60720P, 2006.

[76] Jessica Fridrich, Tomáš Pevný, and Jan Kodovský. Statistically undetectable JPEG steganography: Dead ends challenges, and opportunities. MM and Sec'07 - Proceedings of the Multimedia and Security Workshop 2007, pages 3-14, 2007.

[77] Younhee Kim, Zoran Duric, and Dana Richards. Modified matrix encoding technique for minimal distortion steganography. Lecture Notes in Computer Science (including subseries Lecture Notes in Artificial Intelligence and Lecture Notes in Bioinformatics), 4437 LNCS:314 $327,2007$.

[78] Sivaranjani; and Semi Sara Mani. Edge Adaptive Image Steganography Based On LSB Matching Revisited. IEEE Trans- actions on Information Forensics and Security, pages 5(2):201-214, 2010.

[79] Fangjun Huang, Jiwu Huang, Senior Member, and Yun-qing Shi. New Channel Selection Rule for JPEG Steganography. IEEE Transactions on Information Forensics and Security, 7(August):1181-1191, 2012.

[80] Linjie Guo, Jiangqun Ni, and Yun Qing Shi. An Efficient JPEG Steganographic Scheme Using Uniform Embedding. In In Proceedings of IEEE International Workshop on Information Forensics and Security, WIFS'2012, number December 2012, Costa Adeje, Tenerife, Spain, 2012.

[81] Tomáš Denemark and Jessica Fridrich. Improving Steganographic Security by Synchronizing the Selection Channel. In In Proceedings of the 3rd ACM Workshop on In-formation Hiding and Multimedia Security, IH\&MMSec'2015, Portland, Oregon, USA, 2015.

[82] Vahid Sedighi, Rémi Cogranne, Jessica Fridrich, and Senior Member. Content-Adaptive Steganography by Minimizing Statistical Detectability. IEEE Transactions on Information Forensics and Security, TIFS'2016, pages 221 - 234, 2016.

[83] Yuanfeng Pan, Jiangqun Ni, and Wenkang Su. Improved Uniform Embedding for Efficient JPEG Steganography. In In Proceedings of the International Conference on Cloud Computing and Security, ICCCS(2016, volume 10039 of Part of the Lecture Notes in Computer Science book series (LNCS), Springer, pages 125-133, Nanjing, China, 2016.

[84] Weixiang Li and Kejiang Chen. Defining Joint Distortion for JPEG Steganography. In In Proceedings of the 6th ACM Work- shop on Information Hiding and Multimedia Security, IH\&MMSec'2018, pages 5-16, Innsbruck, Austria, 2018.
[85] Ruohan Meng, Steven G. Rice, Jin Wang, and Xingming Sun. A fusion steganographic algorithm based on faster R-CNN. Computers, Materials and Continua, 55(1):1-16, 2018.

[86] Theo Taburet, Patrick Bas, Wadih Sawaya, and Jessica Fridrich. Natural Steganography in JPEG Domain with a Linear Development Pipeline. IEEE Transactions on Information Forensics and Security, 16:173-186, 2021

[87] Rémi Cogranne, Quentin Giboulot, Patrick Bas, Rémi Cogranne, Quentin Giboulot, Patrick Bas, Minimizing Statistical, Quentin Giboulot, and Patrick Bas. Steganography by Minimizing Statistical Detectability: The cases of JPEG and Color Images. IH and MMSec 2021 - Proceedings of the 2021 ACM Workshop on Information Hiding and Multimedia Security, 2021

[88] Ian Goodfellow, Jean Pouget-Abadie, Mehdi Mirza, Bing Xu, David Warde-Farley, Sherjil Ozair, Aaron Courville, and Yoshua Bengio. Generative adversarial networks. Communications of the ACM, 63(11):139144, 2014.

[89] Diederik P. Kingma and Max Welling. Auto-encoding variational bayes. 2nd International Conference on Learning Representations, ICLR 2014 - Conference Track Proceedings, (Ml):1-14, 2014.

[90] Evgeny Burnaev Denis Volkhonskiy, Boris Borisenko. GENERATIVE ADVERSARIAL NETWORKS FOR IMAGE STEGANOGRAPHY. pages 1-10, 2017.

[91] Haichao Shi, Jing Dong, Wei Wang, and Yinlong Qian. SSGAN: Secure Steganography Based on Generative Adversarial Networks Haichao. Pacific Rim Conference on Multimedia : Advances in Multimedia Information Processing, PCM 2017:534-544, 2017.

[92] Martin Arjovsky, Soumith Chintala, and Léon Bottou. Wasserstein GAN. 2017.

[93] Casey Chu, Andrey Zhmoginov, and Mark Sandler. CycleGAN, a Master of Steganography. (Nips):1-6, 2017.

[94] Weixuan Tang, Shunquan Tan, Bin Li, and Jiwu Huang. Automatic Steganographic Distortion Learning Using a Generative Adversarial Network. IEEE Signal Processing Letters, 24(10):1547-1551, 2017.

[95] Guanshuo Xu, Han Zhou Wu, and Yun Qing Shi. Structural design of convolutional neural networks for steganalysis. IEEE Signal Processing Letters, 23(5):708-712, 2016.

[96] Denis Volkhonskiy, Ivan Nazarov, and Evgeny Burnaev. Steganographic generative adversarial networks. page 97, 2017.

[97] Jamie Hayes and George Danezis. Generating steganographic images via adversarial training. Advances in Neural Information Processing Systems, 2017-Decem:1955-1964, 2017.

[98] Jiren Zhu, Russell Kaplan, Justin Johnson, and Li Fei-fei. HiDDeN:Hiding Data With Deep Networks. 2018

[99] Zhuo Zhang, Guangyuan Fu, Fuqiang Di, Changlong Li, and Jia Liu. Generative Reversible Data Hiding by Image-to-Image Translation via GANs. Security and Communication Networks, 2019, 2019.

[100] Kevin Alex Zhang, Alfredo Cuesta-Infante, Lei Xu, and Kalyan Veeramachaneni. SteganoGAN: High Capacity Image Steganography with GANs. 2019.

[101] Ru Zhang, Shiqi Dong, and Jianyi Liu. Invisible steganography via generative adversarial networks. Multimedia Tools and Applications, 78(7):8559-8575, 2019.

[102] Zhuo Zhang, Guangyuan Fu, Jia Liu, and Wenyu Fu. Generative information hiding method based on adversarial networks, volume 905. Springer International Publishing, 2020.

[103] Zhangjie Fu, Fan Wang, and Xu Cheng. The secure steganography for hiding images via GAN. Eurasip Journal on Image and Video Processing, 2020(1), 2020.

[104] Alec Radford, Luke Metz, and Soumith Chintala. Unsupervised representation learning with deep convolutional generative adversarial networks. 4th International Conference on Learning Representations, ICLR 2016 - Conference Track Proceedings, pages 1-16, 2016.

[105] Neil F. Johnson and Sushil Jajodia. Steganalysis of images created using current steganography software. Lecture Notes in Computer Science (including subseries Lecture Notes in Artificial Intelligence and Lecture Notes in Bioinformatics), 1525:273-289, 1998.

[106] Siwei Lyu and Hany Farid. Detecting Hidden Messages Using HigherOrder Statistics and Support Vector Machines. In 5th International Workshop on Information Hiding, Noordwijkerhout, The Nether- lands, 2002.

[107] Jessica Fridrich. Feature-Based Steganalysis for JPEG Images and its Implications for Future Design of Steganographic Schemes. In International Workshop on Information Hiding, pages 67-81. 2004.

[108] Kenneth Sullivan, Upamanyu Madhow, Shivkumar Chandrasekaran, and B S Manjunath. Steganalysis of Spread Spectrum Data Hiding 
Exploiting Cover Memory. Proc. SPIE 5681, Security, Steganography, and Watermarking of Multimedia Contents VII, 2005.

[109] Nasir Ismail Avcıbas; Kharrazi, Mehdi; Memon. Image Steganalysis with Binary Similarity Measures. EURASIP Journal on Applied Signal Processing 2005:17, pages 2749-2757, 2005.

[110] Dekun Zou, Yun Q Shi, Wei Su, and Guorong Xuan. STEGANALYSIS BASED ON MARKOV MODEL OF THRESHOLDED PREDICTION-ERROR IMAGE ECE Dept ., New Jersey Institute of Technology, Newark, New Jersey, USA. In IEEE International Conference on Multimedia and Expo, pages 1365-1368, 2006.

[111] Jessica Jan Kodovskýa, Tomáš Pevný, Fridrich. Modern Steganalysis Can Detect YASS. In Proc. SPIE 7541, Media Forensics and Security II, 754102, San Jose, California, United States, 2010.

[112] Jessica Fridrich, Jan Kodovský, Vojtěch Holub, and Miroslav Goljan. Steganalysis of Content-Adaptive Steganography in Spatial Domain. In International Workshop on Information Hiding, pages 102-117. 2011.

[113] Jessica Fridrich and Jan Kodovsky. Rich models for steganalysis of digital images. IEEE Transactions on Information Forensics and Security, 7(3):868-882, 2012.

[114] Jan Kodovský and Jessica Fridrich. Steganalysis of JPEG Images Using Rich Models. Proc. SPIE 8303, Media Watermarking, Security, and Forensics, 8303, 2012.

[115] Vojtech Holub and Jessica Fridrich. Random Projections of Residuals for Digital Image Steganalysis. IEEE TRANSACTIONS ON INFORMATION FORENSICS AND SECURITY, 8(12):1996-2006, 2013.

[116] Vojtěch Holub and Jessica Fridrich. Low Complexity Features for JPEG Steganalysis Using Undecimated DCT. IEEE Transactions on Information Forensics and Security, 10(2):219 - 228, 2014.

[117] Vojtěch Holub and Jessica Fridrich. Phase-Aware Projection Model for Steganalysis of JPEG Images. Proc. SPIE 9409, Media Watermarking, Security, and Forensics , 94090T, 2015.

[118] Xiaofeng Song, Fenlin Liu, Chunfang Yang, Xiangyang Luo, and Yi Zhang. Steganalysis of adaptive JPEG steganography using 2D Gabor filters. IH and MMSec 2015 - Proceedings of the 2015 ACM Workshop on Information Hiding and Multimedia Security, pages 1523, jun 2015

[119] Hasan Abdulrahman, Marc Chaumont, Philippe Montesinos, and Baptiste Magnier. Color Image Steganalysis Based on Steerable Gaussian Filters Bank. In IH\&MMSec '16: Proceedings of the 4th ACM Workshop on Information Hiding and Multimedia Security, pages 109114, 2016.

[120] Yi Ma Xia, Chao, Qingxiao Guan, Xianfeng Zhao, Zhoujun Xu. Improving GFR Steganalysis Features by Using Gabor Symmetry and Weighted Histograms. In Proceedings of IH\&MMSec '17, June 20-22, 2017, Philadelphia, PA, USA.

[121] Chao Xia, Keke Wu, Qingxiao Guan, Xinhai Tong, Zhenyu Li, and Yiming Xue. Improved PHARM for JPEG Steganalysis: Making PHARM More Efficient and Effective. IEEE Access, 7:50339-50346, 2019.

[122] Chao Xia, Qingxiao Guan, Xianfeng Zhao, and Keke Wu. Improved JPEG Phase-Aware Steganalysis Features Using Multiple Filter Sizes and Difference Images. IEEE Transactions on Circuits and Systems for Video Technology, 30(11):4100-4113, 2020.

[123] Guorui Feng, Xinpeng Zhang, Yanli Ren, Zhenxing Qian, and Sheng Li. Diversity-Based Cascade Filters for JPEG Steganalysis. IEEE Transactions on Circuits and Systems for Video Technology, 30(2):376386, 2020.

[124] Ismail Avcibas, Nasir D Memon, and Bulent Sankur. Steganalysis Using Image Quality Metrics. IEEE Transactions on Image Processing, 12(February), 2003.

[125] R. R. Salakhutdinov G. E. Hinton. Reducing the Dimensionality of of Data with Neural Networks. Science, 313(July):504-507, 2006.

[126] R. R. Salakhutdinov G. E. Hinton. Reducing the Dimensionality of of Data with Neural Networks. Science, 313(July):504-507, 2006.

[127] Yinlong Qian, Jing Dong, Wei Wang, and Tieniu Tan. Deep learning for steganalysis via convolutional neural networks. Media Watermarking, Security, and Forensics 2015, 9409:94090J, 2015.

[128] Jian Ye, Jiangqun Ni, and Yang Yi. Deep Learning Hierarchical Representations for Image Steganalysis. IEEE Transactions on Information Forensics and Security, 12(11):2545-2557, 2017.

[129] Mo Chen, Mehdi Boroumand, Jessica Fridrich, and Suny Binghamton. Deep Learning Regressors for Quantitative Steganalysis. Electronic Imaging, Media Watermarking, Security, and Forensics 2018, 160:1-7, 2017.

[130] Guanshuo Xu. Deep convolutional neural network to detect JUNIWARD. IH and MMSec 2017 - Proceedings of the 2017 ACM
Workshop on Information Hiding and Multimedia Security, pages 6773, 2017.

[131] Mo Chen, Vahid Sedighi, Mehdi Boroumand, and Jessica Fridrich. JPEG-phase-aware convolutional neural network for steganalysis of JPEG images. IH and MMSec 2017 - Proceedings of the 2017 ACM Workshop on Information Hiding and Multimedia Security, pages 7584, 2017.

[132] Mehdi Yedroudj, Frederic Comby, and Marc Chaumont. Yedroudj-Net: An Efficient CNN for Spatial Steganalysis. ICASSP, IEEE International Conference on Acoustics, Speech and Signal Processing - Proceedings, 2018-April(September):2092-2096, 2018.

[133] Bin Li, Weihang Wei, Anselmo Ferreira, and Shunquan Tan. ReSTNet: Diverse Activation Modules and Parallel Subnets-Based CNN for Spatial Image Steganalysis. IEEE Signal Processing Letters, 25(5):650-654, 2018.

[134] Clement Fuji Tsang and Jessica Fridrich. Steganalyzing images of arbitrary size with CNNs. IS and T International Symposium on Electronic Imaging Science and Technology, pages 1-8, 2018.

[135] Mehdi Boroumand, Mo Chen, and Jessica Fridrich. Deep residual network for steganalysis of digital images. IEEE Transactions on Information Forensics and Security, 14(5):1181-1193, 2019.

[136] Xiaoqing Deng, Bolin Chen, Weiqi Luo, and Da Luo. Fast and effective global covariance pooling network for image steganalysis. IH and MMSec 2019 - Proceedings of the ACM Workshop on Information Hiding and Multimedia Security, pages 230-234, 2019.

[137] Yassine Yousfi and Jessica Fridrich. An Intriguing Struggle of CNNs in JPEG Steganalysis and the OneHot Solution. IEEE Signal Processing Letters, 27(Section IV):830-834, 2020.

[138] Qiangjie Li, Guorui Feng, Hanzhou Wu, and Xinpeng Zhang. Ensemble Steganalysis Based on Deep Residual Network, volume 12022 LNCS. Springer International Publishing, 2020.

[139] Ru Zhang, Feng Zhu, Jianyi Liu, and Gongshen Liu. Depth-Wise Separable Convolutions and Multi-Level Pooling for an Efficient Spatial CNN-Based Steganalysis. IEEE Transactions on Information Forensics and Security, 15:1138-1150, 2020.

[140] Mehdi Yedroudj, Marc Chaumont, Frederic Comby, Ahmed Oulad Amara, and Patrick Bas. Pixels-off: Data-augmentation Complementary Solution for Deep-learning Steganalysis. IH and MMSec 2020 . Proceedings of the 2020 ACM Workshop on Information Hiding and Multimedia Security, pages 39-48, 2020.

[141] Wonhyuk Ahn, Haneol Jang, Seung Hun Nam, In Jae Yu, and Heung Kyu Lee. Local-Source Enhanced Residual Network for Steganalysis of Digital Images. IEEE Access, 8:137789-137798, 2020.

[142] Haneol Jang, Tae Woo Oh, and Kibom Kim. Feature Aggregation Networks for Image Steganalysis. IH and MMSec 2020 - Proceedings of the 2020 ACM Workshop on Information Hiding and Multimedia Security, pages 33-38, jun 2020.

[143] Guiyong Xu, Yang Xu, Sicong Zhang, and Xiaoyao Xie. SFRNet: Feature Extraction-Fusion Steganalysis Network Based on Squeezeand-Excitation Block and RepVgg Block. Security and Communication Networks, 2021, 2021.

[144] Xiaohan Ding, Xiangyu Zhang, Ningning Ma, Jungong Han, Guiguang Ding, and Jian Sun. RepVGG: Making VGG-style ConvNets Great Again. (2017), 2021.

[145] Feng Liu, Xuan Zhou, Xuehu Yan, Yuliang Lu, and Shudong Wang. Image steganalysis via diverse filters and squeeze-and-excitation convolutional neural network. Mathematics, 9(2):1-13, 2021.

[146] Soumik Mondal, Yeo Sze Ling, and Arulmurugan Ambikapathi. HStegonet: A Hybrid Deep Learning Framework for Robust Steganalysis. pages $1-6$, jun 2021.

[147] Yiwei Zhang, Weiming Zhang, Kejiang Chen, Jiayang Liu, Yujia Liu, and Nenghai Yu. Adversarial examples against deep neural network based steganalysis. IH and MMSec 2018 - Proceedings of the 6th ACM Workshop on Information Hiding and Multimedia Security, (June):6772, 2018

[148] DominicBreuker. stego-toolkit: Collection of steganography tools helps with CTF challenges, 2017.

[149] Amazon AWS. Cloud Services - Amazon Web Services (AWS)

[150] Google Cloud. Cloud Computing Services - Google Cloud.

[151] Microsoft Azure. Cloud Computing Services - Microsoft Azure.

[152] Jennifer Newman, Li Lin, Wenhao Chen, Stephanie Reinders, Yangxiao Wang, Min Wu, and Yong Guan. StegoAppDB: A steganography apps forensics image database. IS and T International Symposium on Electronic Imaging Science and Technology, 2019(5), 2019.

[153] Ruiz; Mehdi Yedroudj Hugo, Marc; Fr'ed'eric Comby;Gerard ;Chaumont, and Subsol. LSSD: a Controlled Large JPEG Image Database for Deep-Learning-based Steganalysis. (December 2020), 2021. 
[154] SIPI Image Database. http://sipi.usc.edu/database/ Online; accessed 2021-09-07.

[155] BOWS-2. http://bows2.ec-lille.fr// Online; accessed 2021-09-07.

[156] BOSSBase. http://agents.fel.cvut.cz/boss/index.php?mode=VIEW\& tmpl=materials Online; accessed 2021-09-07.

[157] Dresden. http://forensics.inf.tu-dresden.de/ddimgdb/

[158] Olga Russakovsky, Jia Deng, Hao Su, Jonathan Krause, Sanjeev Satheesh, Sean Ma, Zhiheng Huang, Andrej Karpathy, Aditya Khosla, Michael Bernstein, Alexander C. Berg, and Li Fei-Fei. ImageNet Large Scale Visual Recognition Challenge. International Journal of Computer Vision, 115(3):211-252, dec 2015.

[159] Image Manipulation Dataset. https://www5.cs.fau.de/research/data/ image-manipulation// Online; accessed 2021-09-07.

[160] COCO - Common Objects in Context. https://cocodataset.org//\#home Online; accessed 2021-09-07.

[161] Tsung Yi Lin, Michael Maire, Serge Belongie, James Hays, Pietro Perona, Deva Ramanan, Piotr Dollár, and C. Lawrence Zitnick. Microsoft COCO: Common objects in context. Lecture Notes in Computer Science (including subseries Lecture Notes in Artificial Intelligence and Lecture Notes in Bioinformatics), 8693 LNCS(PART 5):740-755, 2014.

[162] RAISE - The Raw Images Dataset. http://loki.disi.unitn.it/RAISE/ Online; accessed 2021-09-07.

[163] Duc Tien Dang-Nguyen, Cecilia Pasquini, Valentina Conotter, and Giulia Boato. RAISE - A raw images dataset for digital image forensics. Proceedings of the 6th ACM Multimedia Systems Conference, MMSys 2015, pages 219-224, mar 2015.

[164] DIV2K Dataset. https://data.vision.ee.ethz.ch/cvl/DIV2K/ Online; accessed 2021-09-07.

[165] Alaska. https://alaska.utt.fr//\#material Online; accessed 2021-09-07.

[166] Zhongliang Yang, Ke Wang, Sai Ma, Yongfeng Huang, Xiangui Kang, and Xianfeng Zhao. IStego100K: Large-Scale Image Steganalysis Dataset. Lecture Notes in Computer Science (including subseries Lecture Notes in Artificial Intelligence and Lecture Notes in Bioinformatics), 12022 LNCS:352-364, 2020.

[167] Steganalysis for still images with LSB Steganography - Features dataset - IEEE DataPort. https://ieee-dataport.org/open-access/ steganalysis-still-images-lsb-steganography-features-dataset Online; accessed 2021-09-07.

[168] Rémi Cogranne, Quentin Giboulot, and Patrick Bas. The Alaska steganalysis challenge: A first step towards steganalysis "into the wild". IH and MMSec 2019 - Proceedings of the ACM Workshop on Information Hiding and Multimedia Security, pages 125-137, 2019.

[169] LSSD (Large Scale Steganalysis Database. http://www.lirmm.fr/\$ sim\$chaumont/LSSD.html Online; accessed 2021-09-07.

[170] Zhou Wang and Alan C. Bovik. A universal image quality index. IEEE Signal Processing Letters, 9(3):81-84, 2002.

[171] Martin Heusel, Hubert Ramsauer, Thomas Unterthiner, Bernhard Nessler, and Sepp Hochreiter. GANs trained by a two time-scale update rule converge to a local Nash equilibrium. Advances in Neural Information Processing Systems, 2017-December(Nips):66276638, 2017.

[172] Tim Salimans, Ian Goodfellow, Wojciech Zaremba, Vicki Cheung, Alec Radford, and Xi Chen. Improved techniques for training GANs. Advances in Neural Information Processing Systems, pages 2234-2242, 2016.

[173] Xin Yang, Yuezun Li, and Siwei Lyu. Exposing Deep Fakes Using Inconsistent Head Poses. ICASSP, IEEE International Conference on Acoustics, Speech and Signal Processing - Proceedings, 2019May:8261-8265, 2019.

[174] Falko Matern, Christian Riess, and Marc Stamminger. Exploiting visual artifacts to expose deepfakes and face manipulations. Proceedings 2019 IEEE Winter Conference on Applications of Computer Vision Workshops, WACVW 2019, pages 83-92, 2019.

[175] Heidi Wilcox and Maumita Bhattacharya. Countering social engineering through social media: An enterprise security perspective. Lecture Notes in Computer Science (including subseries Lecture Notes in Artificial Intelligence and Lecture Notes in Bioinformatics), 9330 LNCS:54-64, 2015.

[176] Zhi Wang, Chaoge Liu, and Xiang Cui. EvilModel: Hiding Malware Inside of Neural Network Models. pages 1-7, 2021. 